\title{
EXPLICIT BLOCK-STRUCTURES FOR BLOCK-SYMMETRIC FIEDLER-LIKE PENCILS*
}

\author{
M. I. BUENO ${ }^{\dagger}$, M. MARTIN ‡, J. PÉREZ §, A. SONG ף, AND I. VIVIANO ॥
}

\begin{abstract}
In the last decade, there has been a continued effort to produce families of strong linearizations of a matrix polynomial $P(\lambda)$, regular and singular, with good properties, such as, being companion forms, allowing the recovery of eigenvectors of a regular $P(\lambda)$ in an easy way, allowing the computation of the minimal indices of a singular $P(\lambda)$ in an easy way, etc. As a consequence of this research, families such as the family of Fiedler pencils, the family of generalized Fiedler pencils (GFP), the family of Fiedler pencils with repetition, and the family of generalized Fiedler pencils with repetition (GFPR) were constructed. In particular, one of the goals was to find in these families structured linearizations of structured matrix polynomials. For example, if a matrix polynomial $P(\lambda)$ is symmetric (Hermitian), it is convenient to use linearizations of $P(\lambda)$ that are also symmetric (Hermitian). Both the family of GFP and the family of GFPR contain block-symmetric linearizations of $P(\lambda)$, which are symmetric (Hermitian) when $P(\lambda)$ is. Now the objective is to determine which of those structured linearizations have the best numerical properties. The main obstacle for this study is the fact that these pencils are defined implicitly as products of so-called elementary matrices. Recent papers in the literature had as a goal to provide an explicit block-structure for the pencils belonging to the family of Fiedler pencils and any of its further generalizations to solve this problem. In particular, it was shown that all GFP and GFPR, after permuting some block-rows and block-columns, belong to the family of extended block Kronecker pencils, which are defined explicitly in terms of their block-structure. Unfortunately, those permutations that transform a GFP or a GFPR into an extended block Kronecker pencil do not preserve the block-symmetric structure. Thus, in this paper we consider the family of block-minimal bases pencils, which is closely related to the family of extended block Kronecker pencils, and whose pencils are also defined in terms of their block-structure, as a source of canonical forms for block-symmetric pencils. More precisely, we present four families of block-symmetric pencils which, under some generic nonsingularity conditions are block minimal bases pencils and strong linearizations of a matrix polynomial. We show that the block-symmetric GFP and GFPR, after some row and column permutations, belong to the union of these four families. Furthermore, we show that, when $P(\lambda)$ is a complex matrix polynomial, any block-symmetric GFP and GFPR is permutationally congruent to a pencil in some of these four families. Hence, these four families of pencils provide an alternative but explicit approach to the block-symmetric Fiedler-like pencils existing in the literature.
\end{abstract}

Key words. Fiedler pencil, block-symmetric generalized Fiedler pencil, block-symmetric generalized Fiedler pencil with repetition, matrix polynomial, strong linearization, symmetric strong linearization, block Kronecker pencil, extended block Kronecker pencil, block minimal bases pencil

AMS subject classifications. 65F15, 15A18, 15A22, 15A54.

${ }^{*}$ Received by the editors on Date of Submission. Accepted for publication on Date of Acceptance. Handling Editor: Handling Editor.

$\dagger$ Department of Mathematics and College of Creative Studies, University of California, Santa Barbara, CA 93106, USA (mbueno@math.ucsb.edu). The research of M. I. Bueno was partially supported by NSF grant DMS-1358884 and partially supported by Ministerio de Economia y Competitividad of Spain through grants MTM2015-65798-P.

${ }^{\ddagger}$ Brown University Providence (madeline_martin@brown.edu). The research of M. Martin was partially supported by NSF grant DMS-1358884.

$\S$ Department of Mathematical Sciences, University of Montana, MT, USA (javier.perez-alvaro@mso.umt.edu.). The research of J. Pérez was partially supported by KU Leuven Research Council grant OT/14/074 and the Interuniversity Attraction Pole DYSCO, initiated by the Belgian State Science Policy Office.

I University of California, Santa Barbara (alexandersong@umail.ucsb.edu). The research of A. Song was partially supported by NSF grant DMS-1358884.

"Wake Forest University (viviiv14@wfu.edu). The research of I. Viviano was partially supported by NSF grant DMS1358884 . 
1. Introduction. The standard approach to numerically solving a polynomial eigenvalue problem (PEP) associated with a matrix polynomial (whose matrix coefficients have entries in a field $\mathbb{F}$ ) of the form

$$
P(\lambda)=\sum_{i=0}^{k} A_{i} \lambda^{i}, \quad \text { with } A_{0}, A_{1}, \ldots, A_{k} \in \mathbb{F}^{m \times n},
$$

starts by embedding the coefficients of $P(\lambda)$ into a matrix pencil (that is, a matrix polynomial of grade equal to 1). This process is known as linearization, and it transforms the given PEP into a generalized eigenvalue problem (GEP). Then, the obtained GEP can be solved by using the QZ algorithm [24] or the staircase algorithm [27, 28], for example.

The literature on linearizations is huge as can be seen, for example, by counting all the references in [5] concerning this topic. The best well-known examples of linearizations of a matrix polynomial $P(\lambda)$ as in (1.1) are the so-called Frobenius companion forms given by

$$
\left[\begin{array}{cccc}
\lambda A_{k}+A_{k-1} & A_{k-2} & \cdots & A_{0} \\
-I_{n} & \lambda I_{n} & & \\
& \ddots & \ddots & \\
& & -I_{n} & \lambda I_{n}
\end{array}\right] \text { and }\left[\begin{array}{cccc}
\lambda A_{k}+A_{k-1} & -I_{m} & & \\
A_{k-2} & \lambda I_{m} & \ddots & \\
\vdots & & \ddots & -I_{m} \\
A_{0} & & & \lambda I_{m}
\end{array}\right] \text {. }
$$

We note that here and throughout the paper, we sometimes omit the block-entries of a matrix polynomial that are equal to zero as we have done above. The algorithm QZ implemented in Matlab to solve the PEP uses the first Frobenius companion form as a linearization by default.

Frobenius companion forms have many desirable properties from a numerical point of view, as i) they are constructed from the matrix coefficients of $P(\lambda)$ without performing any arithmetic operations; ii) they are strong linearizations of $P(\lambda)$ regardless of whether $P(\lambda)$ is regular or singular [11, 13]; iii) the minimal indices of $P(\lambda)$ are related with the minimal indices of the Frobenius companion forms by uniform shifts [10, 11]; iv) eigenvectors of regular matrix polynomials and minimal bases of singular matrix polynomials are easily recovered from those of the Frobenius companion forms [11]; and v) solving PEP's by applying a backward stable eigensolver to the Frobenius companion forms is backward stable [15, 28]. Nonetheless, solving a PEP by solving the GEP associated with a Frobenius companion form presents some significant drawbacks. For instance, if the matrix polynomial $P(\lambda)$ is symmetric (Hermitian), that is $P(\lambda)^{T}=P(\lambda)(\mathbb{F}=\mathbb{C}$ and $P(\lambda)^{*}=P(\lambda)$ ), neither of the Frobenius companion forms is symmetric (Hermitian). Since the preservation of the structure has been recognized as key for obtaining better (and physically more meaningful) numerical results [22], this drawback has motivated an intense research on structure-preserving linearizations; see, for example $[2,3,4,6,7,12,16,22,25,29]$, to name a few recent references on this topic. There are many papers in the literature addressing the problem of constructing symmetric (Hermitian) strong linearizations of symmetric (Hermitian) matrix polynomials. Most of these papers approach the problem by constructing first block-symmetric strong linearizations, as it is done, for example, in [3, 4, 20, 22].

Among the block-symmetric linearizations in the literature, it has been shown that, within the vector space of block-symmetric pencils $\mathbb{D L}(P)[20,21]$, the first and last pencils in its standard basis, denoted by $D_{1}(\lambda, P)$ and $D_{k}(\lambda, P)$, respectively, have almost optimal behavior in terms of conditioning and backward error when used to compute an eigenvalue $\delta$ of $P(\lambda)$, as long as $|\delta| \geq 1$ if $D_{1}(\lambda, P)$ is used or $|\delta| \leq 1$ if $D_{k}(\lambda, P)$ is used $[19,26]$. A natural question is whether a single block-symmetric linearization can be 
found with good conditioning and backward error regardless of the modulus of $\delta$ or if any block-symmetric linearizations outside $\mathbb{D L}(P)$ present a better numerical behavior than $D_{1}(\lambda, P)$ and $D_{k}(\lambda, P)$. One possible approach to answering these questions consists in replacing some nonzero blocks of the form $\pm A_{i}$ in the matrix coefficients of these pencils (which can be seen as block-matrices whose blocks are of the form 0 , $\pm I_{n}$, and $\pm A_{i}$ ) by zero or identity blocks. But in order to do that, it is necessary to identify which of those blocks are essential to keep a given linearization a linearization of $P(\lambda)$ as they are replaced by zero or identity blocks. Thus, an explicit block-structure of the well-known block-symmetric pencils in the literature that allows to determine easily if they are a linerization of $P(\lambda)$ or not can be useful, for example, to accomplish this goal. In this paper we focus on the block-symmetric pencils in the families of Fiedler-like pencils presented in [3, 4], which are known as block-symmetric generalized Fiedler pencils (block-symmetric GFP) and block-symmetric generalized Fiedler pencils with repetition (block-symmetric GFPR). The block-symmetric GFP are strong linearizations of any $P(\lambda)$. The block-symmetric GFPR are strong linearizations of $P(\lambda)$ modulo some generic nonsingularity conditions. Moreover, all block-symmetric GFP and GFPR are symmetric (Hermitian) when $P(\lambda)$ is. Furthermore, they share some of the desirable properties of the Frobenius companion forms mentioned above. The main disadvantage of these pencils is that they were defined implicitly in terms of products of elementary matrices, which makes it difficult to study their algebraic and numerical properties. Thus, identifying their block structure might solve some of these difficulties.

The family of block minimal bases pencils was recently constructed with the goal of performing a backward stability analysis of PEP's when solved by linearization [15]. These pencils are defined by their explicit block-structure. Moreover, it has been shown that, modulo some generic nonsingularity conditions, Fiedler pencils, generalized Fiedler pencils, Fiedler pencils with repetition (and, thus, the standard basis of the $\mathbb{D L}(P)$ space), and generalized Fiedler pencils with repetition are permutationally equivalent to block minimal bases pencils $[5,15]$. However, none of these results takes into account any extra structural properties that these pencils might possess. For example, given a block-symmetric GFPR, the results in [5, 15] do not guarantee that this pencil is permutationally block-congruent ${ }^{1}$ to a block-symmetric block minimal bases pencil. The focus of this paper is not on constructing new families of block-symmetric pencils but on identifying a family of block-symmetric pencils, that under some generic nonsingularity conditions are block minimal bases pencils, and showing that the block-symmetric GFP and the block-symmetric GFPR are permutationally block-congruent to a pencil in that family. This family of block-symmetric minimal bases pencils can be divided into four subfamilies, two associated with odd degree polynomials and two associated with even degree polynomials. Each of these subfamilies is built by applying certain block-congruences to a very simple block-symmetric block minimal bases pencil, the "skeleton" or "generator" of the family. The "skeleton" of each family contains a "minimal" block-structure (in the sense that its matrix coefficients contain more zero blocks and less nonzero nonidentity blocks than any other pencil in the family) that guarantees it being a strong linearization of a given matrix polynomial $P(\lambda)$. Hence, this approach allows to identify the block-entries of the block-structure of strong linearizations based on block-symmetric Fiedler-like pencils (including the basis of $\mathbb{D L}(P)$ ) that are essential to embed the spectral information of $P(\lambda)$ in the pencil and the block-entries that are not while preserving the block-symmetry. We expect these "skeletons" to be candidates to have optimal numerical properties among the block-symmetric linearizations in the family they "generate".

The rest of the paper is structured as follows. In section 2, we review the basic theory of matrix

\footnotetext{
${ }^{1}$ Given two block-symmetric pencils $L_{1}(\lambda)$ and $L_{2}(\lambda)$, we say that they are permutationally block-congruent if there exists a block-permutation matrix $Q$ such that $L_{1}(\lambda)=Q L_{2}(\lambda) Q^{\mathcal{B}}$, where $M^{\mathcal{B}}$ denotes the block-transpose of the matrix $M$
} 
polynomials, linearizations, minimal bases and dual minimal bases needed throughout the paper. In Section 3, we recall the definitions of the family of block minimal bases pencils and the family of extended block Kronecker pencils. By using extended block Kronecker pencils, we introduce in Section 4 four families of block-symmetric pencils which are block minimal bases pencils under generic nonsingularity conditions, and contain infinitely many block-symmetric strong linearizations of a matrix polynomial. These pencils are explicitly defined in terms of their block-entries. In Section 5, we recall the definitions of block-symmetric GFP and block-symmetric GFPR. Finally, in Section 6 we give a result that states that the block-symmetric GFP associated with an odd degree matrix polynomial and any block-symmetric GFPR is permutationally block-congruent to a pencil belonging to some of the four families introduced in Section 4. Thus, these four families of pencils provide an alternative and simplified approach to block-symmetric Fiedler-like pencils by providing their explicit block-structure. The proof of the result for the block-symmetric GFPR turns out to be quite involved, long and highly technical. One reason for this is that the family of block-symmetric GFPR is infinite and we are stating theorems that hold true for all the pencils in this family. The other reason, as we said before, is that these pencils are defined in an implicit way in terms of products of matrices, which makes the work with them quite cumbersome. The implicit definition of these pencils also leads to the use of a very heavy notation. Since the proof of this result is very similar to the proof of Theorem 8.1 in [5], we include its proof in the Appendix. Now that we have an explicit definition of the block-symmetric GFPR in terms of their block entries, all the notation and the original implicit definition can be abandoned. What remains is a simpler description of block-symmetric Fiedler-like linearizations as block-symmetric block minimal bases pencils. This explicit definition of the block-symmetric GFPR has already proven to be useful. In [8,9], it has been used to identify sparse pencils that outperform numerically (in terms of conditioning and backward error) the block-symmetric linearizations $D_{1}(\lambda, P)$ and $D_{k}(\lambda, P)$ in the standard basis of $\mathbb{D L}(P)$.

2. Notation and background. Throughout the paper, we use the following notation. If $a$ and $b$ are two integers, we define

$$
a: b:= \begin{cases}a, a+1, \ldots, b, & \text { if } a \leq b \\ \emptyset, & \text { if } a>b\end{cases}
$$

In this work we consider square matrix polynomials whose matrix coefficients have entries in a field $\mathbb{F}$, that is, matrix polynomials as in (1.1) with $m=n$. The number $k$ in (1.1) is called the grade of $P(\lambda)$. The degree of $P(\lambda)$ is defined as the largest $d$ such that $A_{d} \neq 0$. Notice that the degree is a number intrinsic to $P(\lambda)$, while the grade is an option (larger than or equal to the degree).

A square matrix polynomial $P(\lambda)$ is said to be regular if the scalar polynomial $\operatorname{det}(P(\lambda))$ is not the zero polynomial; otherwise $P(\lambda)$ is said to be singular. Furthermore, if $\operatorname{det} P(\lambda) \in \mathbb{F}, P(\lambda)$ is called a unimodular matrix polynomial. The complete eigenstructure of a regular matrix polynomial consists of its finite and infinite elementary divisors. For a singular matrix polynomial, the complete eigenstructure consists of its finite and infinite elementary divisors together with its right and left minimal indices. For more detailed definitions of the complete eigenstructure of matrix polynomials, we refer the reader to [14, Section 2].

By the polynomial eigenvalue problem (PEP) associated with a matrix polynomial $P(\lambda)$, we refer to the problem of computing the complete eigenstructure of $P(\lambda)$. If $P(\lambda)$ is a matrix pencil, the associated PEP is referred to as a generalized eigenvalue problem $(G E P)$. A strong linearization of a regular matrix polynomial $P(\lambda)$ is a matrix pencil $\mathcal{L}(\lambda)$ having the same finite and infinite elementary divisors as $P(\lambda)$; when $P(\lambda)$ is singular, a strong linearization must also have the same numbers of right and left minimal indices as $P(\lambda)$. Hence, the PEP associated with the polynomial $P(\lambda)$ can be solved by solving the GEP associated with 
$\mathcal{L}(\lambda)$ provided that the minimal indices of $P(\lambda)$ and $\mathcal{L}(\lambda)$ are related in a simple way.

Given two matrix polynomials $P(\lambda)$ and $Q(\lambda)$ of the same size, we recall the following equivalence relations. The polynomials $P(\lambda)$ and $Q(\lambda)$ are said to be

(i) unimodularly equivalent if there are unimodular matrix polynomials $U(\lambda)$ and $V(\lambda)$ such that $Q(\lambda)=$ $U(\lambda) P(\lambda) V(\lambda)$; and

(ii) strictly equivalent if there are nonsingular constant matrices $U$ and $V$ such that $Q(\lambda)=U P(\lambda) V$.

We recall that unimodular equivalence preserves the finite eigenstructure of matrix polynomials, while strict equivalence preserves the whole eigenstructure [18]. In this work we also use the following concepts extensively.

(i) Given an $s \times t$ block matrix $M=\left[M_{i j}\right]$ with $n \times n$ block-entries $M_{i j}$, the block-transpose matrix of $M$, denoted by $M^{\mathcal{B}}$, is the $t \times s$ block-matrix whose $(i, j)$ block-entry is $M_{j i}$.

(ii) Given a $k \times k$ block matrix $M=\left[M_{i j}\right]$ with $n \times n$ block-entries $M_{i j}$, we say that $M$ is block-symmetric if $M^{\mathcal{B}}=M$.

(iii) A $k n \times k n$ permutation matrix $\Pi$ is called a block-permutation matrix if $\Pi=P \otimes I_{n}$, for some $k \times k$ permutation matrix $P$, where $\otimes$ denotes the Kronecker product of two matrices.

(iv) We say that the matrix polynomials $P(\lambda)$ and $Q(\lambda)$ are permutationally equivalent if there are permutation matrices $\Pi_{1}$ and $\Pi_{2}$ such that $Q(\lambda)=\Pi_{1} P(\lambda) \Pi_{2}$.

(v) We say that the $k n \times k n$ matrix polynomials $P(\lambda)$ and $Q(\lambda)$ are permutationally block-congruent if there exists a block-permutation matrix $\Pi$ such that $Q(\lambda)=\Pi P(\lambda) \Pi^{\mathcal{B}}$.

We notice that permutational equivalence and, thus, permutational block-congruency are particular instances of strict equivalence. Hence, the matrix polynomials $P(\lambda), \Pi P(\lambda) \Pi^{\mathcal{B}}$ and $\Pi_{1} P(\lambda) \Pi_{2}$ have the same eigenstructure (finite, infinite and singular). Furthermore, since the block-entries of any block permutation matrix $\Pi$ are either the zero or the identity matrices, $P(\lambda)$ is block-symmetric if and only if $\Pi P(\lambda) \Pi^{\mathcal{B}}$ is block-symmetric.

Here and thereafter, we denote by $\mathbb{F}[\lambda]^{m \times n}$ the set of $m \times n$ matrix polynomials, by $\mathbb{F}(\lambda)$ the field of rational functions over $\mathbb{F}$ and by $\mathbb{F}(\lambda)^{n}$ the set of $n$-tuplas with entries in $\mathbb{F}(\lambda)$. By $\overline{\mathbb{F}}$ we denote the algebraic closure of $\mathbb{F}$. Any subspace $\mathcal{W} \subseteq \mathbb{F}(\lambda)^{n}$ is called a rational subspace. We recall that any $\mathcal{W} \subseteq \mathbb{F}(\lambda)^{n}$ has bases consisting entirely of vectors with polynomial entries.

Key for this work are the so-called minimal bases and dual minimal bases, introduced by Forney [17]. For their definitions, we rely on the concept of row-degrees vector of an $m \times n$ matrix polynomial $P(\lambda)$, which is a row vector of length $m$ whose $i$ th component is the maximum of the degrees of the entries in the $i$ th row of $P(\lambda)$. For example, the row-degrees vector of the matrix

$$
\left[\begin{array}{ccc}
1 & \lambda^{2} & 1-\lambda \\
0 & 1 & \lambda
\end{array}\right]
$$

is $[2,1]$.

Definition 2.1. Let $\mathcal{W}$ be a rational subspace of $\mathbb{F}(\lambda)^{n}$. We say that a matrix polynomial $L(\lambda) \in$ $\mathbb{F}[\lambda]^{m \times n}$ is a minimal basis of $\mathcal{W}$ if its rows form a basis for $\mathcal{W}$ and the sum of the entries of its row-degrees vector is minimal among all the possible polynomial bases for $\mathcal{W}$. Furthermore, the entries of the row-degrees vector of $L(\lambda)$ are called the minimal indices of $\mathcal{W}$.

REMARK 2.2. For simplicity, we say that " $L(\lambda) \in \mathbb{F}[\lambda]^{m \times n}$ is a minimal basis" to mean that " $L(\lambda)$ is a 
minimal basis for the subspace of $\mathbb{F}(\lambda)^{n}$ spanned by its rows".

The following characterization of minimal bases is very useful in practice.

TheOREm 2.3. Let $L(\lambda) \in \mathbb{F}[\lambda]^{m \times n}$ and let $\left[d_{1}, \ldots, d_{m}\right]$ be the row-degrees vector of $L(\lambda)$. Then, $L(\lambda)$ is a minimal basis if and only if $L\left(\lambda_{0}\right)$ has full row rank for all $\lambda_{0} \in \overline{\mathbb{F}}$ and the $m \times n$ constant matrix whose $(i, j)$ th entry is the coefficient of $\lambda^{d_{j}}$ in the $(i, j)$ th entry of $L(\lambda)$ has full row rank.

EXAMPLE 2.4. The matrix polynomial in (2.2) is a minimal basis because it clearly has full row rank for every $\lambda_{0} \in \overline{\mathbb{F}}$ and the matrix $\left[\begin{array}{lll}0 & 1 & 0 \\ 0 & 0 & 1\end{array}\right]$ has full row rank as well.

Definition 2.5. Two matrix polynomials $L(\lambda) \in \mathbb{F}[\lambda]^{m_{1} \times n}$ and $N(\lambda) \in \mathbb{F}[\lambda]^{m_{2} \times n}$ are called dual minimal bases if $m_{1}+m_{2}=n, L(\lambda) N(\lambda)^{T}=0$, and $L(\lambda)$ and $N(\lambda)$ are both minimal bases.

REMARK 2.6. We will say that "N( $\lambda)$ is a minimal basis dual to $L(\lambda)$ ", or vice versa, when referring to matrix polynomials $L(\lambda)$ and $N(\lambda)$ as those in Definition 2.5 .

Continuing with the example in (2.2), it is easy to show that the matrix polynomials

$$
\left[\begin{array}{ccc}
1 & \lambda^{2} & 1-\lambda \\
0 & 1 & \lambda
\end{array}\right] \text { and }\left[\begin{array}{lll}
\lambda^{3}+\lambda-1 & -\lambda & 1
\end{array}\right]
$$

are dual minimal bases.

In the following proposition, we introduce the most important pair of dual minimal bases used in this work.

Proposition 2.7. [15] Let

$$
L_{s}(\lambda):=\left[\begin{array}{ccccc}
-1 & \lambda & & & \\
& -1 & \lambda & & \\
& & \ddots & \ddots & \\
& & & -1 & \lambda
\end{array}\right] \in \mathbb{F}[\lambda]^{s \times(s+1)},
$$

and

$$
\Lambda_{s}(\lambda):=\left[\begin{array}{llll}
\lambda^{s} & \cdots & \lambda & 1
\end{array}\right] \in \mathbb{F}[\lambda]^{1 \times(s+1)} .
$$

Then, for every positive integer $p$, the matrix polynomials $L_{s}(\lambda) \otimes I_{p}$ and $\Lambda_{s}(\lambda) \otimes I_{p}$ are dual minimal bases.

The following proposition concerning dual minimal bases will be useful.

Proposition 2.8. Let $L(\lambda)$ be a minimal basis. If $B$ is a nonsingular matrix, then $B L(\lambda)$ is also a minimal basis. Further, if $N(\lambda)$ is any minimal basis dual to $L(\lambda), N(\lambda)$ is also dual to $B L(\lambda)$.

Proof. The proof follows immediately from the characterization of minimal bases in Theorem 2.3, and the definition of dual minimal bases in Definition 2.5.

3. Block minimal bases pencils and extended block Kronecker pencils. We recall in this section the familis of block minimal bases pencils and of extended block Kronecker pencils, and state their main properties used in this work. 
3.1. Block minimal bases pencils. The block minimal bases pencils were introduced in [15]. The definition of block minimal bases pencil involves the concept of minimal basis and pair of dual minimal bases introduced in the previous section.

Definition 3.1. A matrix pencil

$$
C(\lambda)=\left[\begin{array}{c|c}
M(\lambda) & G_{2}(\lambda)^{T} \\
\hline G_{1}(\lambda) & 0
\end{array}\right]
$$

is called a block minimal bases pencil if $G_{1}(\lambda)$ and $G_{2}(\lambda)$ are both minimal bases. If, in addition, the rowdegrees vector of $G_{1}(\lambda)$ (resp. $G_{2}(\lambda)$ ) have all entries equal to 1 and the entries of the row-degrees vector of a minimal basis dual to $G_{1}(\lambda)$ (resp. $G_{2}(\lambda)$ ) are all equal, then $C(\lambda)$ is called a strong block minimal bases pencil.

A fundamental property of any strong block minimal bases pencil of the form (3.5) is that it is a strong linearization of some matrix polynomial expressed in terms of the block-entry $M(\lambda)$ and the dual minimal bases of $G_{1}(\lambda)$ and $G_{2}(\lambda)$.

Theorem 3.2. [15, Theorem 3.3] Let $C(\lambda)$ be a strong block minimal bases pencil as in (3.5). Let $N_{1}(\lambda)$ (resp. $N_{2}(\lambda)$ ) be a minimal basis dual to $G_{1}(\lambda)$ (resp. $G_{2}(\lambda)$ ) whose row-degrees vector has equal entries. Let

$$
Q(\lambda):=N_{2}(\lambda) M(\lambda) N_{1}(\lambda)^{T} .
$$

Then, $C(\lambda)$ is a strong linearization of $Q(\lambda)$, considered as a polynomial of grade $1+\operatorname{deg}\left(N_{1}(\lambda)\right)+\operatorname{deg}\left(N_{2}(\lambda)\right)$.

3.2. Extended block Kronecker pencils. Next we recall a family of pencils that has played an important role in the canonical expression of the GFP and GFPR in terms of their block-structure [5]. The pencils in this family are called extended block Kronecker pencils. In their definition, we use the dual minimal bases $L_{s}(\lambda)$ and $\Lambda_{s}(\lambda)$ introduced, respectively, in (2.3) and (2.4).

DeFinition 3.3. [5, Definition 3.5] Let $M(\lambda)$ be an arbitrary $(q+1) m \times(p+1) n$ pencil. Let $A \in \mathbb{F}^{n p \times n p}$ and $B \in \mathbb{F}^{m q \times m q}$ be arbitrary matrices. Then the matrix pencil

$$
C(\lambda)=\left[\begin{array}{c|c}
M(\lambda) & \left(L_{q}(\lambda)^{T} \otimes I_{m}\right) B \\
\hline A(\underbrace{\left.L_{p}(\lambda) \otimes I_{n}\right)}_{(p+1) n} & 0 \\
\underbrace{}_{q m} & \begin{array}{l}
\}(q+1) m \\
p n
\end{array}
\end{array}\right.
$$

where $L_{p}(\lambda)$ and $L_{q}(\lambda)$ are as in (2.3), is called an extended $(p, n, q, m)$-block Kronecker pencil or, simply, an extended block Kronecker pencil. When $A=I_{n p}$ and $B=I_{m q}$, then $C(\lambda)$ is called a block Kronecker pencil. The block $M(\lambda)$ is called the body of $C(\lambda)$.

Note that, if $A$ and $B$ are nonsingular matrices, then $C(\lambda)$ is a (strong) block minimal bases pencil (see Proposition 2.8). However, if either $A$ or $B$ is singular, it is not guaranteed that $C(\lambda)$ is a block minimal bases pencil.

One advantage of the extended block Kronecker pencils with $A$ and $B$ nonsingular over more general strong block minimal bases pencils is that it is easy to give simple characterizations for all the grade- 1 solutions $M(\lambda)$ of the equation

$$
\underset{7}{\left(\Lambda_{q}(\lambda)^{T} \otimes I_{m}\right) M(\lambda)\left(\Lambda_{p}(\lambda) \otimes I_{n}\right)=P(\lambda),}
$$


for a prescribed matrix polynomial $P(\lambda)$ of grade $k=p+q+1$.

The following definition will be used in one of such characterizations.

Definition 3.4. [5, Definition 3.7] Let $M(\lambda)=\lambda M_{1}+M_{0} \in \mathbb{F}[\lambda]^{(q+1) m \times(p+1) n}$ be a matrix pencil and set $k:=p+q+1$. Let us denote by $\left[M_{0}\right]_{i j}$ and $\left[M_{1}\right]_{i j}$ the $(i, j)$ th block-entries of $M_{0}$ and $M_{1}$, respectively, when $M_{0}$ and $M_{1}$ are partitioned as $(q+1) \times(p+1)$ block-matrices with blocks of size $m \times n$. We call the antidiagonal sum of $M(\lambda)$ related to $s \in\{0: k\}$ the matrix

$$
\operatorname{AS}(M, s):=\sum_{i+j=k+2-s}\left[M_{1}\right]_{i j}+\sum_{i+j=k+1-s}\left[M_{0}\right]_{i j} .
$$

Additionally, given a matrix polynomial $P(\lambda)=\sum_{i=0}^{k} A_{i} \lambda^{i} \in \mathbb{F}[\lambda]^{m \times n}$, we say that $M(\lambda)$ satisfies the antidiagonal sum condition (AS condition) for $P(\lambda)$ if

$$
\operatorname{AS}(M, s)=A_{s}, \quad s=0: k .
$$

The AS condition has been used in the construction of large classes of linearizations of a matrix polynomial $P(\lambda)$ easily constructible from the coefficients of $P(\lambda)$; see [15, Theorem 5.4] or [16, Section 3].

Example 3.5. Let $P(\lambda)=\sum_{i=0}^{5} A_{i} \lambda^{i}$. The matrix pencil

$$
M(\lambda)=\left[\begin{array}{ccc}
A_{5} \lambda & 0 & 0 \\
A_{4} \lambda & 0 & 0 \\
A_{3} \lambda & A_{2} \lambda & A_{1} \lambda+A_{0}
\end{array}\right] .
$$

satisfies the AS condition for $P(\lambda)$.

TheOREM 3.6. Let $P(\lambda)=\sum_{i=0}^{k} A_{i} \lambda^{i} \in \mathbb{F}[\lambda]^{m \times n}$, and let $C(\lambda)$ be an extended block Kronecker pencil as in (3.7) with $p+q+1=k$ and with body $M(\lambda)$. The following conditions are equivalent.

(a) The pencil $M(\lambda)$ satisfies (3.8).

(b) The pencil $M(\lambda)$ satisfies the $A S$ condition for $P(\lambda)$.

(c) The pencil $M(\lambda)$ is of the form

$$
M(\lambda)=M_{0}(\lambda)+C_{1}\left(L_{p}(\lambda) \otimes I_{n}\right)+\left(L_{q}(\lambda)^{T} \otimes I_{m}\right) C_{2},
$$

where $M_{0}(\lambda)$ is any solution of (3.8) and $C_{1} \in \mathbb{F}^{(q+1) m \times p n}$ and $C_{2} \in \mathbb{F}^{q m \times(p+1) n}$ are arbitrary matrices.

Proof. The proof that (a) and (b) are equivalent can be obtained by some simple algebraic manipulations. The proof that parts (a) and (c) are equivalent can be found in [16] (in a paragraph just before Theorem $1)$.

Now, as an immediate consequence of Theorem 3.6, we obtain the following family of strong linearizations of $P(\lambda)$.

THEOREM 3.7. Let $P(\lambda)$ be a matrix polynomial, and let $p, q$ be nonnegative integers such that $p+q+1=$ $\operatorname{deg}(P(\lambda))$. Let $M_{0}(\lambda)$ be a pencil satisfying the $A S$ condition for $P(\lambda)$. Then, any pencil of the form

$$
\left[\begin{array}{c|c}
M_{0}(\lambda)+C_{1}\left(L_{p}(\lambda) \otimes I_{n}\right)+\left(L_{q}(\lambda)^{T} \otimes I_{m}\right) C_{2} & \left(L_{q}(\lambda)^{T} \otimes I_{m}\right) B_{2} \\
\hline B_{1}\left(L_{p}(\lambda) \otimes I_{n}\right)_{8} & 0
\end{array}\right],
$$


where $C_{1} \in \mathbb{F}^{(q+1) m \times p n}$ and $C_{2} \in \mathbb{F}^{q m \times(p+1) n}$ are arbitrary matrices, and $B_{1} \in \mathbb{F}^{p n \times p n}$ and $B_{2} \in \mathbb{F}^{q m \times q m}$ are arbitrary nonsingular matrices, is a strong linearization of $P(\lambda)$.

Proof. The result is an immediate consequence of Theorems 3.2 and 3.6, together with the fact that, when the matrices $B_{1}$ and $B_{2}$ are nonsingular, the extended block Kronecker pencil (3.10) is a strong block minimal bases pencil (see Proposition 2.8).

REMARK 3.8. Observe that any pencil of the form (3.10) is an extended block Kronecker pencil whose body satisfies the $A S$ condition for $P(\lambda)$ since, given two matrix pencils $M_{1}(\lambda)$ and $M_{2}(\lambda), \operatorname{AS}\left(M_{1}+M_{2}, s\right)=$ $\operatorname{AS}\left(M_{1}, s\right)+\operatorname{AS}\left(M_{2}, s\right)$. Moreover, the pencil in (3.10) can be expressed as follows:

$$
\left[\begin{array}{ll}
I & C_{1} \\
0 & B_{1}
\end{array}\right]\left[\begin{array}{c|c}
M_{0}(\lambda) & L_{q}(\lambda)^{T} \otimes I_{m} \\
\hline L_{p}(\lambda) \otimes I_{n} & 0
\end{array}\right]\left[\begin{array}{cc}
I & 0 \\
C_{2} & B_{2}
\end{array}\right] .
$$

Theorem 3.7 will be key to provide a simple canonical block-structure for block-symmetric Fiedler-like pencils under permutational block-congruence operations. The description of these block-structures is the main goal of the following section.

4. The four families of block-symmetric minimal bases pencils. We introduce in this section four types of block-symmetric pencils associated with a matrix polynomial $P(\lambda)$, which are block minimal bases pencils, under some generic nonsingularity conditions, and we give their explicit block structure. We will show later that the block-symmetric Fiedler-like pencils known in the literature belong to one of these families, modulo a permutational block-congruence.

Since block-symmetric Fiedler-like pencils are only defined for square matrix polynomials, here and thereafter, we restrict our study to square matrix polynomials $P(\lambda) \in \mathbb{F}[\lambda]^{n \times n}$. As the size of $P(\lambda)$ is always going to be denoted by $n$, there is no risk of confusion if we introduce the notation

$$
K_{s}(\lambda):=L_{s}(\lambda) \otimes I_{n}
$$

with $L_{s}(\lambda)$ as in (2.3). We note that

$$
K_{s}(\lambda)^{T}=K_{s}(\lambda)^{\mathcal{B}} \quad \text { and } \quad\left(\Lambda_{s}(\lambda) \otimes I_{n}\right)^{T}=\left(\Lambda_{s}(\lambda) \otimes I_{n}\right)^{\mathcal{B}}
$$

with $\Lambda_{s}(\lambda)$ as in (2.4), when $K_{s}(\lambda)$ is seen as an $s \times(s+1)$ block matrix with blocks of size $n \times n$ and $\Lambda_{s}(\lambda) \otimes I_{n}$ is seen as a $1 \times(s+1)$ block matrix with blocks of size $n \times n$. Moreover, if $B$ is an $s \times s$ block matrix, then

$$
\left(B K_{s}(\lambda)\right)^{\mathcal{B}}=K_{s}(\lambda)^{\mathcal{B}} B^{\mathcal{B}}=K_{s}(\lambda)^{T} B^{\mathcal{B}}
$$

Additionally, we introduce the block-symmetric pencil

$$
M(\lambda ; Q):=\left[\begin{array}{lll}
\lambda Q_{d}+Q_{d-1} & & \\
& \ddots & \\
& & \lambda Q_{1}+Q_{0}
\end{array}\right] \in \mathbb{F}[\lambda]^{\frac{n(d+1)}{2} \times \frac{n(d+1)}{2}}
$$

associated with a matrix polynomial $Q(\lambda)=\sum_{i=0}^{d} Q_{i} \lambda^{i} \in \mathbb{F}[\lambda]^{n \times n}$ of odd degree $d$, which will play a fundamental role in what follows. Notice that $M(\lambda ; Q)$ satisfies the AS condition for $Q(\lambda)$. 
Associated with the matrix polynomial $P(\lambda)=\sum_{i=0}^{k} A_{i} \lambda^{i} \in \mathbb{F}[\lambda]^{n \times n}$, we define the following matrix polynomials

$$
\begin{aligned}
& P^{k-1}(\lambda):=A_{k-1} \lambda^{k-1}+\cdots+\lambda A_{1}+A_{0}, \\
& P_{k-1}^{k-1}(\lambda):=A_{k-1} \lambda^{k-2}+\cdots+A_{2} \lambda+A_{1}, \quad \text { and } \\
& P_{k-1}(\lambda):=A_{k} \lambda^{k-1}+\cdots+\lambda A_{2}+A_{1},
\end{aligned}
$$

which will be used in the definition of the four families of block-symmetric pencils introduced in this section. Note that $P^{k-1}(\lambda)$ is a truncation of degree $k-1$ of $P(\lambda)$ while $P_{k-1}(\lambda)$ is the so-called $(k-1)$ th Horner shift polynomial associated with $P(\lambda)$.

4.1. The first fundamental block-structure. We introduce here the first of the families of blocksymmetric pencils. Let $P(\lambda) \in \mathbb{F}[\lambda]^{n \times n}$ be a matrix polynomial of odd degree $k$, and let $s:=(k-1) / 2$. We start by defining the pencil

$$
\mathcal{O}_{1}^{P}(\lambda):=\left[\begin{array}{c|c}
M(\lambda ; P) & K_{s}(\lambda)^{T} \\
\hline K_{s}(\lambda) & 0
\end{array}\right] \in \mathbb{F}[\lambda]^{n k \times n k}
$$

where $M(\lambda ; P)$ is defined in (4.13). By Definition 3.3, the pencil $\mathcal{O}_{1}^{P}(\lambda)$ is an $(s, n, s, n)$ - block Kronecker pencil and a strong block minimal bases pencil. Furthermore, taking into account (4.11), it is clearly block-symmetric. Notice additionally that, by Theorem 3.7, $\mathcal{O}_{1}^{P}(\lambda)$ is a strong linearization of $P(\lambda)$ because $M(\lambda ; P)$ satisfies the AS condition for $P(\lambda)$. Thus, the pencil $\mathcal{O}_{1}^{P}(\lambda)$ is a block-symmetric strong linearization of $P(\lambda)$.

We can obtain many more block-symmetric strong linearizations of $P(\lambda)$ by considering pencils obtained by applying the block-congruence

$$
\left[\begin{array}{cc}
I_{(s+1) n} & C \\
0 & B
\end{array}\right]\left[\begin{array}{c|c}
M(\lambda ; P) & K_{s}(\lambda)^{T} \\
\hline K_{s}(\lambda) & 0
\end{array}\right]\left[\begin{array}{cc}
I_{(s+1) n} & 0 \\
C^{\mathcal{B}} & B^{\mathcal{B}}
\end{array}\right],
$$

where $B=\left[B_{i j}\right]$ is an $s \times s$ block matrix and $C=\left[C_{i j}\right]$ is an $(s+1) \times s$ block-matrix, with $n \times n$ block-entries $B_{i j}$ and $C_{i j}$, respectively. The pencil (4.18) motivates the first fundamental block-structure family associated with the matrix polynomial $P(\lambda)$.

Definition 4.1. Let $P(\lambda)=\sum_{i=0}^{k} A_{i} \lambda^{i} \in \mathbb{F}[\lambda]^{n \times n}$ be a matrix polynomial of odd degree $k$, and let $s=(k-1) / 2$. The first fundamental block-structure family, denoted by $\left\langle\mathcal{O}_{1}^{P}\right\rangle$, is the set of pencils of the form

$$
\left[\begin{array}{c|c}
M(\lambda ; P)+C K_{s}(\lambda)+K_{s}^{T}(\lambda) C^{\mathcal{B}} & K_{s}(\lambda)^{T} B^{\mathcal{B}} \\
\hline B K_{s}(\lambda) & 0
\end{array}\right],
$$

where $M(\lambda ; P)$ is defined in (4.13), and $B=\left[B_{i j}\right]$ and $C=\left[C_{i j}\right]$ are, respectively, some arbitrary $s \times s$ block matrix and $(s+1) \times s$ block matrix, with $n \times n$ block-entries $B_{i j}$ and $C_{i j}$.

REMARK 4.2. The matrix pencil in (4.19), which is also the pencil in (4.18), can be expressed as follows:

$$
\left[\begin{array}{cc}
I_{(s+1) n} & C \\
0 & B
\end{array}\right]\left[\begin{array}{c|c}
M(\lambda ; P) & K_{s}(\lambda)^{T} \\
\hline K_{s}(\lambda) & 0
\end{array}\right]\left[\begin{array}{cc}
I_{(s+1) n} & C \\
0 & B
\end{array}\right]^{\mathcal{B}},
$$


where the block transpose is applied on the matrix $\left[\begin{array}{cc}I_{(s+1) n} & C \\ 0 & B\end{array}\right]$ when considered a $k \times k$ block matrix. That is, every pencil in $\left\langle\mathcal{O}_{1}^{P}\right\rangle$ is block congruent to $\mathcal{O}_{1}^{P}$ and, therefore, block-symmetric.

By (4.11) and Definition 3.3, any pencil in the family $\left\langle\mathcal{O}_{1}^{P}\right\rangle$ is a block-symmetric $(s, n, s, n)$-extended block Kronecker pencil. Moreover, if $B$ and $B^{\mathcal{B}}$ are nonsingular, each pencil in this family is a strong block minimal bases pencil, which leads to the following theorem.

Theorem 4.3. Let $P(\lambda)=\sum_{i=0}^{k} A_{i} \lambda^{i} \in \mathbb{F}[\lambda]^{n \times n}$ be a matrix polynomial of odd degree $k$, let $s=$ $(k-1) / 2$, and let $\mathcal{L}(\lambda) \in\left\langle\mathcal{O}_{1}^{P}\right\rangle$, that is, $\mathcal{L}(\lambda)$ is of the form (4.19). If $B$ and $B^{\mathcal{B}}$ are nonsingular, then the pencil $\mathcal{L}(\lambda)$ is a block-symmetric strong linearization of $P(\lambda)$. Moreover, if $P(\lambda)$ and all the block-entries $B_{i j}$ are symmetric (resp. Hermitian), then the pencil $\mathcal{L}(\lambda)$ is symmetric (resp. Hermitian).

Proof. The fact that $\mathcal{L}(\lambda)$ is a strong linearization of $P(\lambda)$ when $B$ and $B^{\mathcal{B}}$ are nonsingular is an immediate consequence of Theorem 3.7. The pencil $\mathcal{L}(\lambda)$ is block-symmetric as a consequence of (4.11), together with the fact that $M(\lambda ; P)$ is block-symmetric. The fact that $\mathcal{L}(\lambda)$ is symmetric (resp. Hermitian) when $P(\lambda)$ and all the block-entries $B_{i j}$ of $B$ are symmetric (resp. Hermitian) follows easily from the facts that $M(\lambda ; P)$ is symmetric (resp. Hermitian) when $P(\lambda)$ is symmetric (resp. Hermitian), and that $B^{\mathcal{B}}=B^{T}\left(B^{\mathcal{B}}=B^{*}\right)$ and $C^{\mathcal{B}}=C^{T}\left(C^{\mathcal{B}}=C^{*}\right)$ when all the block-entries $B_{i j}$ and $C_{i j}$ are symmetric (resp. Hermitian).

EXAMPLE 4.4. As mentioned in the introduction, the best well-known block-symmetric pencils in the literature are those in the vector space $\mathbb{D L}(P)$. The pencils in the standard basis of this space are blocksymmetric GFPR of special importance. Let $P(\lambda)$ be a matrix polynomial of odd degree $k$ and let $m$ be an odd positive integer. Then, as we will show in Theorem 6.2, the mth pencil $D_{m}(\lambda, P)$ in the standard basis of $\mathbb{D L}(P)$, which is a GFPR with parameter $h=k-m$, is permutationally block-congruent to a pencil in $\left\langle\mathcal{O}_{1}^{P}\right\rangle$. This holds, in particular, for $D_{1}(\lambda, P)$ and $D_{k}(\lambda, P)$.

4.2. The second fundamental block-structure. We introduce in this section the second fundamental family of block-symmetric pencils. This family is also associated with odd-degree matrix polynomials, but describing its block-structure is more involved.

Let $P(\lambda)=\sum_{i=0}^{k} A_{i} \lambda^{i}$ be an $n \times n$ matrix polynomial of odd degree $k$, and let $s:=(k-1) / 2$. First, we define the pencil

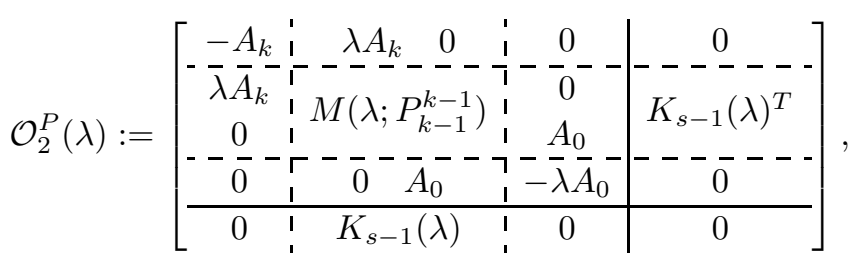

where $P_{k-1}^{k-1}(\lambda)$ is defined in $(4.15)$ and $M\left(\lambda ; P_{k-1}^{k-1}\right)$ is defined in (4.13). Notice that the pencil $\mathcal{O}_{2}^{P}(\lambda)$ is a block-symmetric block minimal bases pencil. However, this pencil is not an extended block-Kronecker pencil.

Next we give an example to clarify the block-structure of the pencil $\mathcal{O}_{2}^{P}(\lambda)$. 
ExAmple 4.5. Let $P(\lambda)=\sum_{i=0}^{7} A_{i} \lambda^{i} \in \mathbb{F}[\lambda]^{n \times n}$. Then,

$$
\mathcal{O}_{2}^{P}(\lambda)=\left[\begin{array}{c:ccc:c|cc}
-A_{7} & \lambda A_{7} & 0 & 0 & 0 & 0 & 0 \\
\hdashline \lambda A_{7} & \lambda A_{6}+A_{5} & 0 & 0 & 0 & -I_{n} & 0 \\
0 & 0 & \lambda A_{4}+A_{3} & 0 & 0 & \lambda I_{n} & -I_{n} \\
0 & 0 & 0 & \lambda A_{2}+A_{1} & A_{0} & 0 & \lambda I_{n} \\
\hdashline 0 & 0 & 0 & A_{0} & -\lambda A_{0} & 0 & 0 \\
\hdashline 0 & -I_{n} & \lambda I_{n} & 0 & 0 & 0 & 0 \\
0 & 0 & -I_{n} & \lambda I_{n} & 0 & 0 & 0
\end{array}\right] .
$$

Notice that, if we denote by $\Pi_{2}$ the block-permutation matrix that permutes the first block-column of $\mathcal{O}_{2}^{P}$ with the block-columns in positions 2-5, we have

$$
\begin{aligned}
\mathcal{O}_{2}^{P}(\lambda) \Pi_{2}= & {\left[\begin{array}{c|cc|ccc}
M(\lambda) & K_{3}(\lambda)^{T} B_{2} \\
\hline B_{1} K_{3}(\lambda) & 0
\end{array}\right]:=} \\
& {\left[\begin{array}{ccccccc}
\lambda A_{7} & 0 & 0 & 0 & -A_{7} & 0 & 0 \\
\lambda A_{6}+A_{5} & 0 & 0 & 0 & \lambda A_{7} & -I_{n} & 0 \\
0 & \lambda A_{4}+A_{3} & 0 & 0 & 0 & \lambda I_{n} & -I_{n} \\
0 & 0 & \lambda A_{2}+A_{1} & A_{0} & 0 & 0 & \lambda I_{n} \\
\hline 0 & 0 & A_{0} & -\lambda A_{0} & 0 & 0 & 0 \\
-I_{n} & \lambda I_{n} & 0 & 0 & 0 & 0 & 0 \\
0 & -I_{n} & \lambda I_{n} & 0 & 0 & 0 & 0
\end{array}\right], }
\end{aligned}
$$

where

$$
B_{1}=\left[\begin{array}{ccc}
0 & 0 & -A_{0} \\
I_{n} & 0 & 0 \\
0 & I_{n} & 0
\end{array}\right] \quad \text { and } \quad B_{2}=\left[\begin{array}{ccc}
A_{7} & 0 & 0 \\
0 & I_{n} & 0 \\
0 & 0 & I_{n}
\end{array}\right] .
$$

Thus, although $\mathcal{O}_{2}^{P}(\lambda)$ is not an extended block Kronecker pencil, it is only a column-permutation away from being so. It is easy to see that the body $M(\lambda)$ of $\mathcal{O}_{2}^{P}(\lambda) \Pi_{2}$ satisfies the AS condition for $P(\lambda)$. Hence, by Theorem 3.7 and Remark 3.8, the pencil $\mathcal{O}_{2}^{P}(\lambda) \Pi_{2}$, and therefore $\mathcal{O}_{2}^{P}(\lambda)$, is a strong linearization of $P(\lambda)$ if $A_{0}$ and $A_{k}$ are nonsingular matrices.

The procedure used in the previous example can be generalized to matrix polynomials of any odd-degree $k$. Denoting by $\Pi_{2}$ the block-permutation matrix that permutes the first block-column of $\mathcal{O}_{2}^{P}(\lambda)$, defined in (4.20), with the block-columns in positions 2 through $s+2=\frac{k+3}{2}$, we obtain

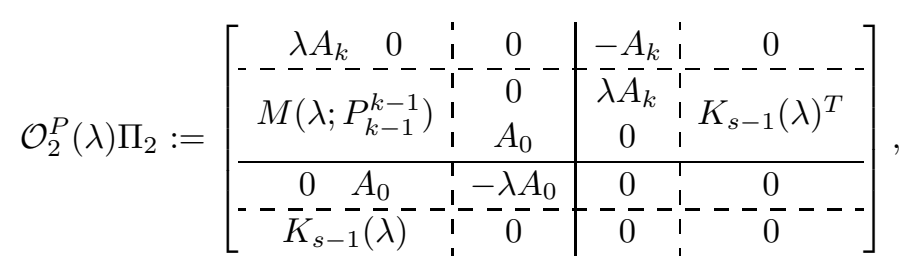

which is an $(s, n, s, n)$-extended block Kronecker pencil. Furthermore, if $A_{0}$ and $A_{k}$ are nonsingular, from Theorem 3.7, Remark 3.8, and the fact that

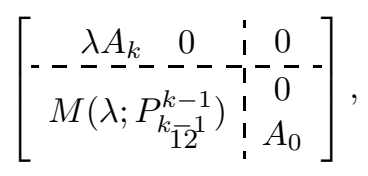


satisfies the AS condition for $P(\lambda)$, it is immediately obtained that the pencil in (4.21) is a strong linearization of $P(\lambda)$. In summary, the pencil $\mathcal{O}_{2}^{P}(\lambda)$ is a block-symmetric strong linearization of $P(\lambda)$ if $A_{0}$ and $A_{k}$ are nonsingular. Moreover, $\mathcal{O}_{2}^{P}(\lambda)$ is symmetric (resp. Hermitian) whenever $P(\lambda)$ is symmetric (resp. Hermitian).

Motivated by the block-structure of the pencil (4.21) and by Theorem 3.7, we now consider a subfamily of extended block Kronecker pencils constructed from $\mathcal{O}_{2}^{P}(\lambda) \Pi_{2}$. Note that, among all the possible operations that would transform $\mathcal{O}_{2}^{P}(\lambda) \Pi_{2}$ into another extended block Kronecker pencil, we are only applying some that will preserve the block-symmetry once the $(s+2)$ th block column is permuted back to the original position, that is, the first block-column. More precisely, we begin by considering pencils of the form

$$
\left[\begin{array}{cccc}
I_{n} & 0 & 0 & B \\
0 & I_{s n} & 0 & C \\
0 & 0 & I_{n} & D \\
0 & 0 & 0 & E
\end{array}\right] \mathcal{O}_{2}^{P}(\lambda) \Pi_{2}\left[\begin{array}{cccc}
I_{s n} & 0 & 0 & 0 \\
0 & I_{n} & 0 & 0 \\
0 & 0 & I_{n} & 0 \\
C^{\mathcal{B}} & D^{\mathcal{B}} & B^{\mathcal{B}} & E^{\mathcal{B}}
\end{array}\right]
$$

for some arbitrary $1 \times(s-1), s \times(s-1), 1 \times(s-1)$ and $(s-1) \times(s-1)$ block matrices $B=\left[B_{i j}\right], C=\left[C_{i j}\right]$, $D=\left[D_{i j}\right]$ and $E=\left[E_{i j}\right]$, with $n \times n$ block-entries $B_{i j}, C_{i j}, D_{i j}$ and $E_{i j}$.

Then, permuting the $(s+2)$ th block-column of the above pencil back to the first position, we get

$$
\left[\begin{array}{cccc}
I_{n} & 0 & 0 & B \\
0 & I_{s n} & 0 & C \\
0 & 0 & I_{n} & D \\
0 & 0 & 0 & E
\end{array}\right] \mathcal{O}_{2}^{P}(\lambda)\left[\begin{array}{cccc}
0 & 0 & I_{n} & 0 \\
I_{s n} & 0 & 0 & 0 \\
0 & I_{n} & 0 & 0 \\
C^{\mathcal{B}} & D^{\mathcal{B}} & B^{\mathcal{B}} & E^{\mathcal{B}}
\end{array}\right] \Pi_{2}^{\mathcal{B}}
$$

which equals

$$
\left[\begin{array}{cccc}
I_{n} & 0 & 0 & B \\
0 & I_{s n} & 0 & C \\
0 & 0 & I_{n} & D \\
0 & 0 & 0 & E
\end{array}\right] \mathcal{O}_{2}^{P}(\lambda)\left[\begin{array}{cccc}
I_{n} & 0 & 0 & B \\
0 & I_{s n} & 0 & C \\
0 & 0 & I_{n} & D \\
0 & 0 & 0 & E
\end{array}\right]^{\mathcal{B}}
$$

In this way, we obtain the block-structure (4.23) defining the second fundamental family of block-structures associated with the matrix polynomial $P(\lambda)$.

Definition 4.6. Let $P(\lambda)=\sum_{i=0}^{k} A_{i} \lambda^{i} \in \mathbb{F}[\lambda]^{n \times n}$ be a matrix polynomial of odd degree $k$, let $s=$ $(k-1) / 2$. The second fundamental block-structure family, denoted by $\left\langle\mathcal{O}_{2}^{P}\right\rangle$, is the set of pencils of the form (we are omitting the dependence on $\lambda$ in the pencil $K_{s-1}(\lambda)$ for lack of space)

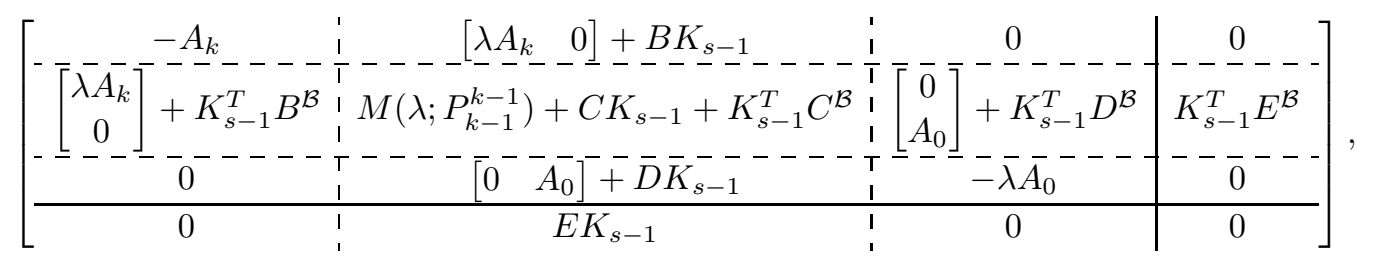

where $P_{k-1}^{k-1}(\lambda)$ is defined in (4.15) and $M\left(\lambda ; P_{k-1}^{k-1}\right)$ is defined in (4.13), for some arbitrary $1 \times(s-1)$ blockmatrix $B=\left[B_{i j}\right], s \times(s-1)$ block-matrix $C=\left[C_{i j}\right], 1 \times(s-1)$ block-matrix $D=\left[D_{i j}\right]$, and $(s-1) \times(s-1)$ block-matrix $E=\left[E_{i j}\right]$, with $n \times n$ block-entries $B_{i j}, C_{i j}, D_{i j}$ and $E_{i j}$, respectively. 
We note that every pencil in $\left\langle\mathcal{O}_{2}^{P}\right\rangle$ is a block minimal bases pencil if $E$ and $E^{\mathcal{B}}$ are nonsingular matrices.

The following theorem gives sufficient conditions for pencils in the family $\left\langle\mathcal{O}_{2}^{P}\right\rangle$ to be strong linearizations of an odd-degree matrix polynomial $P(\lambda)$.

Theorem 4.7. Let $P(\lambda)=\sum_{i=0}^{k} A_{i} \lambda^{i} \in \mathbb{F}[\lambda]^{n \times n}$ be a matrix polynomial of odd degree $k$, let $s=$ $(k-1) / 2$, and consider a pencil $\mathcal{L}(\lambda) \in\left\langle\mathcal{O}_{2}^{P}\right\rangle$, that is, a pencil of the form (4.24). If $A_{0}, A_{k}$, $E$ and $E^{\mathcal{B}}$ are nonsingular, then $\mathcal{L}(\lambda)$ is a block-symmetric strong linearization of $P(\lambda)$. Furthermore, if $P(\lambda)$, and all the block-entries of $B, C, D$ and $E$ are symmetric (resp. Hermitian), then $\mathcal{L}(\lambda)$ is symmetric (resp. Hermitian).

Proof. When $A_{0}$ and $A_{k}$ are nonsingular, the extended block Kronecker pencil (4.21) and, thus, the pencil $\mathcal{O}_{2}^{P}(\lambda)$ are strong linearizations of $P(\lambda)$. In addition, we see from (4.23) that if $E$ and $E^{\mathcal{B}}$ are nonsingular, then the pencil $\mathcal{L}(\lambda)$ is strictly equivalent to the pencil $\mathcal{O}_{2}^{P}(\lambda)$. Therefore, in this case, $\mathcal{L}(\lambda)$ is a strong linearization of $P(\lambda)$. The pencil $\mathcal{L}(\lambda)$ is block-symmetric as a consequence of (4.11), together with the fact that $M\left(\lambda ; P_{k-1}^{k-1}\right)$ is block-symmetric. The fact that $\mathcal{L}(\lambda)$ is symmetric (resp. Hermitian) when $P(\lambda)$ and all the block-entries of $B, C, D$ and $E$ are symmetric (resp. Hermitian) follows easily from the following facts. First, $M\left(\lambda ; P_{k-1}^{k-1}\right)$ is symmetric (resp. Hermitian) when $P(\lambda)$ is symmetric (resp. Hermitian). Secondly, we have $B^{\mathcal{B}}=B^{T}\left(B^{\mathcal{B}}=B^{*}\right), C^{\mathcal{B}}=C^{T}\left(C^{\mathcal{B}}=C^{*}\right), D^{\mathcal{B}}=D^{T}\left(D^{\mathcal{B}}=D^{*}\right)$ and $E^{\mathcal{B}}=E^{T}\left(E^{\mathcal{B}}=E^{*}\right)$ when all the block-entries of $B, C, D$ and $E$ are symmetric (resp. Hermitian).

EXAMPLE 4.8. Let $P(\lambda)$ be a matrix polynomial of odd degree $k$ and let $m$ be an even positive integer. Then, as we will show in Theorem 6.2, the mth pencil $D_{m}(\lambda, P)$ in the standard basis of the vector space $\mathbb{D L}(P)$, which is a GFPR with parameter $h=k-m$, is permutationally block congruent to a pencil in $\left\langle\mathcal{O}_{2}^{P}\right\rangle$.

4.3. The third fundamental block-structure. The third fundamental family of block-symmetric pencils is defined for matrix polynomials of even degree. So, let $P(\lambda)$ be a matrix polynomial of even degree $k$, and let $s:=(k-2) / 2$. First, we define the pencil

$$
\mathcal{E}_{1}^{P}(\lambda):=\left[\begin{array}{c|c:c}
M\left(\lambda ; P_{k-1}\right) & 0 & A_{0}(\lambda)^{T} \\
\hdashline 0 A_{0} & -\lambda A_{0} & 0 \\
\hline K_{s}(\lambda) & 0 & 0
\end{array}\right],
$$

where $P_{k-1}(\lambda)$ is defined in (4.16) and $M\left(\lambda ; P_{k-1}\right)$ is defined in (4.13). The pencil $\mathcal{E}_{1}^{P}(\lambda)$ is an extended $(s, n, s+1, n)$-block Kronecker pencil ${ }^{2}$, with the solid lines indicating one of its natural partitions.

Note that the body of $\mathcal{E}_{1}^{P}(\lambda)$, regardless of the chosen partition (see [15, Theorem 3.10]), satisfies the AS condition for $P(\lambda)$. Thus, $\mathcal{E}_{1}^{P}(\lambda)$ is a strong linearization of $P(\lambda)$, provided that $A_{0}$ is nonsingular. Furthermore, the pencil $\mathcal{E}_{1}^{P}(\lambda)$ is block-symmetric, and it is symmetric (resp. Hermitian) when $P(\lambda)$ is symmetric (resp. Hermitian).

\footnotetext{
${ }^{2}$ It can be seen as an extended $(s+1, n, s, n)$-block Kronecker pencil as well.
} 
ExAmple 4.9. Let $P(\lambda)=\sum_{i=0}^{6} A_{i} \lambda^{i} \in \mathbb{F}[\lambda]^{n \times n}$. Then,

$$
\mathcal{E}_{1}^{P}(\lambda)=\left[\begin{array}{ccc|c:cc}
\lambda A_{6}+A_{5} & 0 & 0 & 0 & -I_{n} & 0 \\
0 & \lambda A_{4}+A_{3} & 0 & 0 & \lambda I_{n} & -I_{n} \\
0 & 0 & \lambda A_{2}+A_{1} & A_{0} & 0 & \lambda I_{n} \\
\hdashline-\bar{c}-A_{0} & 0 & 0 \\
\hline-I_{n} & \lambda I_{n} & 0 & 0 & 0 & 0 \\
0 & -I_{n} & \lambda I_{n} & 0 & 0 & 0
\end{array}\right]
$$

Motivated by the block-structure of the pencil $\mathcal{E}_{1}^{P}(\lambda)$, we introduce the third fundamental family of block-structures by applying the following block-congruence

$$
\left[\begin{array}{ccc}
I_{(s+1) n} & 0 & B \\
0 & I_{n} & C \\
0 & 0 & D
\end{array}\right] \mathcal{E}_{1}^{P}(\lambda)\left[\begin{array}{ccc}
I_{(s+1) n} & 0 & 0 \\
0 & I_{n} & 0 \\
B^{\mathcal{B}} & C^{\mathcal{B}} & D^{\mathcal{B}}
\end{array}\right]
$$

where $B=\left[B_{i j}\right]$ is a $(s+1) \times s$ block-matrix, $C=\left[C_{i j}\right]$ is an $1 \times s$ block-matrix and $D=\left[D_{i j}\right]$ is an $s \times s$ block-matrix, with $n \times n$ block-entries $B_{i j}, C_{i j}$ and $D_{i j}$, respectively.

Definition 4.10. Let $P(\lambda)=\sum_{i=0}^{k} A_{i} \lambda^{i} \in \mathbb{F}[\lambda]^{n \times n}$ be a matrix polynomial of even degree $k$, and let $s=(k-2) / 2$. The third fundamental block-structure family, denoted by $\left\langle\mathcal{E}_{1}^{P}\right\rangle$, is the set of pencils of the form

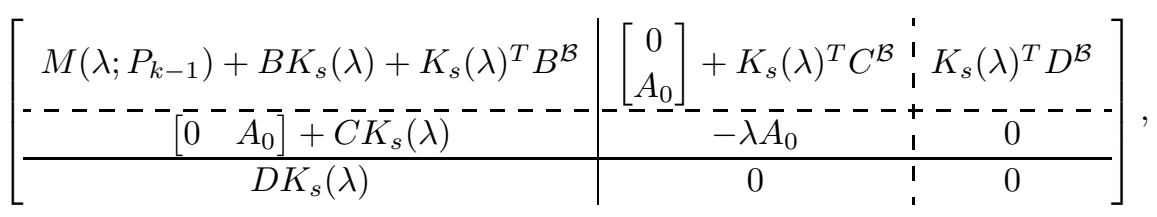

where $P_{k-1}(\lambda)$ is defined in (4.16) and $M\left(\lambda ; P_{k-1}\right)$ is defined in (4.13), for some arbitrary $(s+1) \times s$ blockmatrix $B=\left[B_{i j}\right], 1 \times s$ block-matrix $C=\left[C_{i j}\right]$ and $s \times s$ block-matrix $D=\left[D_{i j}\right]$, with $n \times n$ block-entries $B_{i j}, C_{i j}$ and $D_{i j}$, respectively.

Note that the pencils in $\left\langle\mathcal{E}_{1}^{P}\right\rangle$ are block minimal bases pencils if $A_{0}, D$ and $D^{\mathcal{B}}$ are nonsingunlar.

The following theorem gives sufficient conditions for the pencils in the family $\left\langle\mathcal{E}_{1}^{P}\right\rangle$ to be strong linearizations of the even-degree matrix polynomial $P(\lambda)$.

ThEOREM 4.11. Let $P(\lambda)$ be a matrix polynomial of even degree $k$, let $s=(k-2) / 2$, and consider a pencil $\mathcal{L}(\lambda) \in\left\langle\mathcal{E}_{1}^{P}\right\rangle$ of the form (4.27). If $A_{0}, D$ and $D^{\mathcal{B}}$ are nonsingular, then $\mathcal{L}(\lambda)$ is a block-symmetric strong linearization of $P(\lambda)$. Moreover, if $P(\lambda)$ and all the block-entries $B_{i j}, C_{i j}$ and $D_{i j}$ are symmetric (resp. Hermitian), then $\mathcal{L}(\lambda)$ is symmetric (resp. Hermitian).

Proof. If $A_{0}$ is nonsingular, the pencil $\mathcal{E}_{1}^{P}(\lambda)$ is a strong linearization of $P(\lambda)$. Additionally, if $D$ and $D^{\mathcal{B}}$ are nonsingular, the pencil $\mathcal{L}(\lambda)$ is strictly equivalent to $\mathcal{E}_{1}^{P}(\lambda)$ (see (4.26)). Thus, if $A_{0}, D$ and $D^{\mathcal{B}}$ are nonsingular, $\mathcal{L}(\lambda)$ is a strong linearization of $P(\lambda)$. The fact that $\mathcal{L}(\lambda)$ is block-symmetric follows readily from (4.11) and the fact that $M\left(\lambda ; P_{k-1}\right)$ is block-symmetric. Finally, the fact that $\mathcal{L}(\lambda)$ is symmetric (resp. Hermitian) when $P(\lambda)$ and all the block-entries $B_{i j}, C_{i j}$ and $D_{i j}$ are symmetric (resp. Hermitian) follows easily from the following facts. First, $M\left(\lambda ; P_{k-1}\right)$ is symmetric (resp. Hermitian) when $P(\lambda)$ is symmetric (resp. Hermitian). Secondly, we have $B^{\mathcal{B}}=B^{T}\left(B^{\mathcal{B}}=B^{*}\right), C^{\mathcal{B}}=C^{T}\left(C^{\mathcal{B}}=C^{*}\right)$ and $D^{\mathcal{B}}=D^{T}\left(D^{\mathcal{B}}=D^{*}\right)$ when all the block-entries $B_{i j}, C_{i j}$ and $D_{i j}$ are symmetric (resp. Hermitian). 
EXAMPLE 4.12. Let $P(\lambda)$ be a matrix polynomial of even degree $k$ and let $m$ be an odd positive integer. Then, as we will show in Theorem 6.2, the mth pencil $D_{m}(\lambda, P)$ in the standard basis of the vector space $\mathbb{D L}(P)$, which is a block-symmetric GFPR with parameter $h=k-m$, is permutationally block congruent to a pencil in $\left\langle\mathcal{E}_{1}^{P}\right\rangle$. This holds true, in particular, for $D_{1}(\lambda, P)$.

4.4. The fourth fundamental block-structure. The fourth fundamental block-structure family is also associated with even-degree matrix polynomials. So, let $P(\lambda)$ be a matrix polynomial of even degree $k$, and let $s:=(k-2) / 2$. First, we define the pencil

$$
\mathcal{E}_{2}^{P}(\lambda):=\left[\begin{array}{c:c|c}
-A_{k} & \lambda A_{k}-0 & 0 \\
\hdashline \lambda \bar{A}_{k} & M\left(\lambda ; P^{k-1}\right) & K_{s}(\lambda)^{T} \\
0 & K_{s}(\lambda) & 0
\end{array}\right],
$$

where $P^{k-1}(\lambda)$ is defined in (4.14) and $M\left(\lambda ; P^{k-1}\right)$ is defined in (4.13). By applying a block columnpermutation $\Pi_{4}$ to $\mathcal{E}_{2}^{P}(\lambda)$, we obtain the pencil

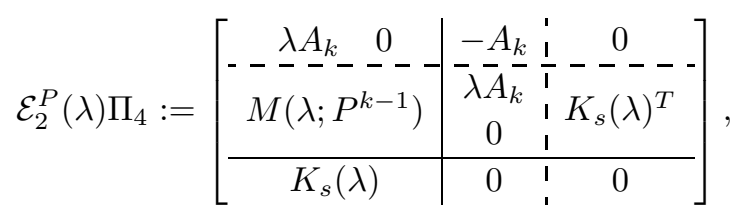

which is an extended $(s, n, s+1, n)$-block Kronecker pencil. Notice that the body of the pencil $\mathcal{E}_{2}^{P}(\lambda) \Pi_{4}$ satisfies the AS condition for $P(\lambda)$. Hence, $\mathcal{E}_{2}^{P}(\lambda) \Pi_{4}$ and, thus, $\mathcal{E}_{2}^{P}(\lambda)$, are strong linearizations of $P(\lambda)$ if $A_{k}$ is nonsingular. Moreover, the pencil $\mathcal{E}_{2}^{P}(\lambda)$ is block-symmetric, and it is symmetric (resp. Hermitian) provided that $P(\lambda)$ is symmetric (resp. Hermitian).

EXAMPLE 4.13. Let $P(\lambda)=\sum_{i=0}^{6} A_{i} \lambda^{i} \in \mathbb{F}[\lambda]^{n \times n}$. Then,

$$
\mathcal{E}_{2}^{P}(\lambda)=\left[\begin{array}{cccc|cc}
-A_{6} & \lambda A_{6} & 0 & 0 & 0 & 0 \\
\lambda A_{6} & \lambda A_{5}+A_{4} & 0 & 0 & -I_{n} & 0 \\
0 & 0 & \lambda A_{3}+A_{2} & 0 & \lambda I_{n} & -I_{n} \\
0 & 0 & 0 & \lambda A_{1}+A_{0} & 0 & \lambda I_{n} \\
\hline 0 & -I_{n} & \lambda I_{n} & 0 & 0 & 0 \\
0 & 0 & -I_{n} & \lambda I_{n} & 0 & 0
\end{array}\right] .
$$

By permuting the first block-column with the block-columns in positions 2-4, we get

$$
\mathcal{E}_{2}^{P}(\lambda) \Pi_{4}=\left[\begin{array}{ccc|ccc}
\lambda A_{6} & 0 & 0 & -A_{6} & 0 & 0 \\
\lambda A_{5}+A_{4} & 0 & 0 & \lambda A_{6} & -I_{n} & 0 \\
0 & \lambda A_{3}+A_{2} & 0 & 0 & \lambda I_{n} & -I_{n} \\
0 & 0 & \lambda A_{1}+A_{0} & 0 & 0 & \lambda I_{n} \\
\hline-I_{n} & \lambda I_{n} & 0 & 0 & 0 & 0 \\
0 & -I_{n} & \lambda I_{n} & 0 & 0 & 0
\end{array}\right],
$$

which is clearly an extended block Kronecker pencil. 
Inspired by the block-structure of $\mathcal{E}_{2}^{P}(\lambda) \Pi_{4}$, we consider extended block Kronecker pencils of the form

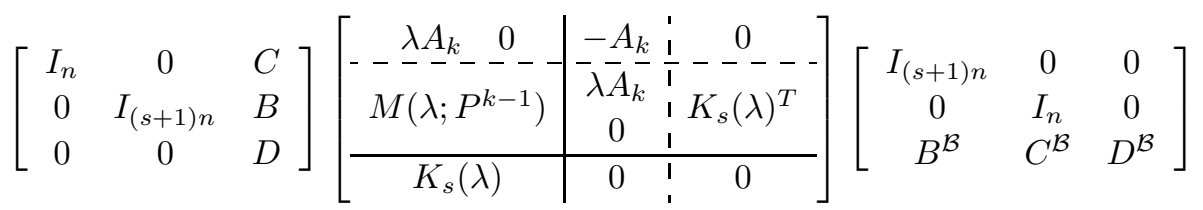

for arbitrary matrices $C \in \mathbb{F}^{n \times s n}, B \in \mathbb{F}^{(s+1) n \times s n}$ and $D \in \mathbb{F}^{s n \times s n}$, or, equivalently,

$$
\left[\begin{array}{c|c:c}
{\left[\lambda A_{k}\right.} & 0]+C K_{s}(\lambda) & 0 \\
\hdashline M\left(\lambda ; P^{k-1}\right)+B K_{s}(\lambda)+K_{s}(\lambda)^{T} B^{\mathcal{B}} & {\left[\begin{array}{c}
\lambda \bar{A} \\
0
\end{array}\right]+K_{s}(\lambda)^{T} C^{\mathcal{B}}} & K_{s}(\lambda)^{T} D^{\mathcal{B}} \\
\hline D K_{s}(\lambda) & 0 & 0
\end{array}\right] .
$$

Reversing the block-permutation we did originally, we obtain the block-structure defining the fourth family of block-structures.

Definition 4.14. Let $P(\lambda)=\sum_{i=0}^{k} A_{i} \lambda^{i} \in \mathbb{F}[\lambda]^{n \times n}$ be a matrix polynomial of even degree $k$, and let $s=(k-2) / 2$. The fourth fundamental block-structure family, denoted by $\left\langle\mathcal{E}_{2}^{P}\right\rangle$, is the set of pencils of the form

$$
\left[\begin{array}{c:c|c}
-A_{k} & {\left[\lambda A_{k}\right.} & 0]+C K_{s}(\lambda) \\
\hdashline\left[\begin{array}{c}
\lambda \overline{A_{k}} \\
0
\end{array}\right]+K_{s}(\lambda)^{T} C^{\mathcal{B}} & M\left(\lambda ; P^{k-1}\right)+B K_{s}(\lambda)+K_{s}(\lambda)^{T} B^{\mathcal{B}} & K_{s}(\lambda)^{T} D^{\mathcal{B}} \\
\hline 0 & D K_{s}(\lambda) & 0
\end{array}\right]
$$

where $P^{k-1}(\lambda)$ is defined in (4.14) and $M\left(\lambda ; P^{k-1}\right)$ is defined in (4.13), for an arbitrary $(s+1) \times s$ blockmatrix $B=\left[B_{i j}\right], 1 \times n$ block-matrix $C=\left[C_{i j}\right]$, and $s \times s$ block-matrix $D=\left[D_{i j}\right]$, with $n \times n$ block entries $B_{i j}, C_{i j}$ and $D_{i j}$, respectively.

Note that all pencils in $\left\langle\mathcal{E}_{2}^{P}\right\rangle$ are block minimal bases pencils if $A_{k}, D$, and $D^{\mathcal{B}}$ are nonsingular.

The following theorem gives necessary and sufficient conditions for pencils in the family $\left\langle\mathcal{E}_{2}^{P}\right\rangle$ to be strong linearizations of the even-degree matrix polynomial $P(\lambda)$.

TheOrem 4.15. Let $P(\lambda)$ be a matrix polynomial of even degree $k$, let $s=(k-2) / 2$, consider a pencil $\mathcal{L}(\lambda) \in\left\langle\mathcal{E}_{2}^{P}\right\rangle$ of the form (4.30). If $A_{k}, D$ and $D^{\mathcal{B}}$ are nonsingular, then $\mathcal{L}(\lambda)$ is a block-symmetric strong linearization of $P(\lambda)$. Moreover, if $P(\lambda)$ and all the block-entries $B_{i j}, C_{i j}$ and $D_{i j}$ are symmetric (resp. Hermitian), then $\mathcal{L}(\lambda)$ is symmetric (resp. Hermitian).

Proof. If $A_{k}$ is nonsingular, the pencil $\mathcal{E}_{2}^{P}(\lambda)$ is a strong linearization of $P(\lambda)$. In addition, notice from (4.29) that if $D$ and $D^{\mathcal{B}}$ are nonsingular, the pencil $\mathcal{L}(\lambda)$ is strictly equivalent to $\mathcal{E}_{2}^{P}(\lambda)$. Thus, if $A_{k}, D$ and $D^{\mathcal{B}}$ are nonsingular, then $\mathcal{L}(\lambda)$ is a strong linearization of $P(\lambda)$. The fact that $\mathcal{L}(\lambda)$ is block-symmetric follows easily from (4.12) and the fact that $M\left(\lambda ; P^{k-1}\right)$ is block-symmetric. Finally, notice the following two facts. First, if $P(\lambda)$ is symmetric (resp. Hermitian) so is $M\left(\lambda ; P^{k-1}\right)$. Secondly, when all the block-entries of $B, C$ and $D$ are symmetric (resp. Hermitian.), we have $B^{\mathcal{B}}=B^{T}$ (resp. $B^{\mathcal{B}}=B^{*}$ ), $C^{\mathcal{B}}=C^{T}$ (resp. $\left.C^{\mathcal{B}}=C^{*}\right)$ and $D^{\mathcal{B}}=D^{T}$ (resp. $D^{\mathcal{B}}=D^{*}$ ). Hence, if $P(\lambda)$ and all the block-entries $B_{i j}, C_{i j}$ and $D_{i j}$ are symmetric (resp. Hermitian), then $\mathcal{L}(\lambda)$ is symmetric (resp. Hermitian). 
EXAMPLE 4.16. Let $P(\lambda)$ be a matrix polynomial of even degree $k$ and let $m$ be an even positive integer. Then, as we will show in Theorem 6.2, the mth pencil $D_{m}(\lambda, P)$ in the standard basis of the vector space $\mathbb{D L}(P)$, which is a block-symmetric GFPR with parameter $h=k-m$, is permutationally block congruent to a pencil in $\left\langle\mathcal{E}_{2}^{P}\right\rangle$. This holds true, in particular, for $D_{k}(\lambda, P)$.

5. Block-symmetric generalized Fiedler pencils and block-symmetric generalized Fiedler pencils with repetition. We introduce in this section the block-symmetric generalized Fiedler pencils and the family of block-symmetric generalized Fiedler pencils with repetition. We start with some concepts and basic results needed for those definitions.

5.1. The index tuple notation and matrix assignments. We start by introducing the fundamental definition of an index tuple and some related notions.

Definition 5.1. [4, Definition 3.1] We call an index tuple a finite ordered sequence of integer numbers. Each of these integers is called an index of the tuple. The number of indices in an index tuple $\mathbf{t}$ is called its length and is denoted by $|\mathbf{t}|$. For integers $a$ and $b$, we call the tuple $(a: b)$ a string.

We will use the following notation for some important basic operations with tuples. If $\mathbf{t}=\left(t_{1}, \ldots, t_{r}\right)$ is an index tuple, we denote $-\mathbf{t}:=\left(-t_{1}, \ldots,-t_{r}\right)$, and, when $a$ is an integer, we denote $a+\mathbf{t}:=\left(a+t_{1}, a+\right.$ $\left.t_{2}, \ldots, a+t_{r}\right)$. We call the reversal index tuple of $\mathbf{t}$ the index tuple $\operatorname{rev}(\mathbf{t}):=\left(t_{r}, \ldots, t_{2}, t_{1}\right)$. Additionally, given index tuples $\mathbf{t}_{1}, \ldots, \mathbf{t}_{s}$, we denote by $\left(\mathbf{t}_{1}, \ldots, \mathbf{t}_{s}\right)$ the index tuple obtained by concatenating the indices in the index tuples $\mathbf{t}_{1}, \ldots, \mathbf{t}_{s}$ in the indicated order.

An important property of index tuples used to define the block-symmetric GFPR is the so-called Successor Infix Property, which we introduce in the following definition.

Definition 5.2. [29, Definition 7] Let $\mathbf{t}=\left(i_{1}, i_{2}, \ldots, i_{r}\right)$ be an index tuple of either all nonnegative integers or all negative integers. Then, $\mathbf{t}$ is said to satisfy the Successor Infix Property (SIP) if for every pair of indices $i_{a}, i_{b} \in \mathbf{t}$, with $1 \leq a<b \leq r$, satisfying $i_{a}=i_{b}$, there exists at least one index $i_{c}=i_{a}+1$ with $a<c<b$.

REMARK 5.3. We note the following basic properties of tuples satisfying the SIP. Any subtuple of consecutive indices of a tuple satisfying the SIP also satisfies the SIP. The reversal of any tuple satisfying the SIP also satisfies the SIP. If the tuple $\mathbf{t}$ has no repeated indices, then $\mathbf{t}$ satisfies the SIP.

The following definitions are motivated by the construction of block-symmetric GFPR in Section 5. For more details, we refer the reader to [2, Section 4] and [7].

Definition 5.4. [2, Definition 4.3] Let $h$ be a nonnegative integer, and let $p=0$ if $h$ is even, and $p=1$ is $h$ is odd. Then, we call the index tuple

$$
\mathbf{w}_{h}:=(h-1: h, h-3: h-2, \ldots, p+1: p+2,0: p)
$$

the admissible tuple associated with the integer $h \geq 0$.

Notice that the tuple $\mathbf{w}_{h}$ is a permutation of the tuple $(0: h)$.

Definition 5.5. [2, Definition 4.3] Let $h$ be a nonnegative integer, and let $\mathbf{w}_{h}$ be the admissible tuple associated with $h$. Then, the symmetric complement of $\mathbf{w}_{h}$ is the tuple

- $\mathbf{c}_{h}:=(h-1, h-3, \ldots, 2,0)$ if $h$ is odd; 
- $\mathbf{c}_{h}:=(h-1, h-3, \ldots, 1)$ if $h>0$ is even;

- $\mathbf{c}_{h}:=\emptyset$ is $h=0$.

Lemma 5.6. [3, Lemma 3.11] Let $h$ be a nonnegative integer, let $\mathbf{w}_{h}$ be the admissible tuple associated with $h$, and let $\mathbf{c}_{h}$ be the symmetric complement of $\mathbf{w}_{h}$. Then, the index tuple $\left(\mathbf{w}_{h}, \mathbf{c}_{h}\right)$ satisfies the SIP.

The matrix coefficients of the block-symmetric GFPR (and that we review in this section) are products of elementary block-matrices, whose definition we recall next.

Definition 5.7. [4] Let $k \geq 2$ be an integer and let $B$ be an arbitrary $n \times n$ matrix. We call elementary matrices the following $k \times k$ block-matrices partitioned into blocks of size $n \times n$ :

$$
\begin{aligned}
M_{0}(B) & :=\left[\begin{array}{c|c}
I_{(k-1) n} & 0 \\
\hline 0 & B
\end{array}\right], \quad M_{-k}(B):=\left[\begin{array}{c|c}
B & 0 \\
\hline 0 & I_{(k-1) n}
\end{array}\right], \\
M_{i}(B) & :=\left[\begin{array}{c|cc|c}
I_{(k-i-1) n} & 0 & 0 & 0 \\
\hline 0 & B & I_{n} & 0 \\
0 & I_{n} & 0 & 0 \\
\hline 0 & 0 & 0 & I_{(i-1) n}
\end{array}\right], \quad i=1: k-1, \\
M_{-i}(B) & :=\left[\begin{array}{c|cc|c}
I_{(k-i-1) n} & 0 & 0 & 0 \\
\hline 0 & 0 & I_{n} & 0 \\
0 & I_{n} & B & 0 \\
\hline 0 & 0 & 0 & I_{(i-1) n}
\end{array}\right] \quad i=1: k-1,
\end{aligned}
$$

and

$$
M_{-0}(B):=M_{0}(B)^{-1} \quad \text { and } \quad M_{k}(B):=M_{-k}(B)^{-1} .
$$

assuming that $B$ is nonsingular.

Notice that the notation -0 does not have the usual meaning, that is, in this case $-0 \neq 0$.

REMARK 5.8. Notice that, for $i=1: k-1$, the elementary matrices $M_{i}(B)$ and $M_{-i}(B)$ are nonsingular for any $B$. Furthermore, $\left(M_{i}(B)\right)^{-1}=M_{-i}(-B)$. On the other hand, the matrices $M_{0}(B)$ and $M_{-k}(B)$ are nonsingular if and only if $B$ is nonsingular.

Definition 5.9. [2, Definition 4.6] Let $\mathbf{t}=\left(i_{1}, i_{2}, \ldots, i_{r}\right)$ be an index tuple with indices contained in $\{-k: k-1\}$ and let $\mathcal{Z}=\left(Z_{1}, \ldots, Z_{r}\right)$ be a list of $r$ arbitrary $n \times n$ matrices. We define

$$
M_{\mathbf{t}}(\mathcal{Z}):=M_{i_{1}}\left(Z_{1}\right) M_{i_{2}}\left(Z_{2}\right) \cdots M_{i_{r}}\left(Z_{r}\right)
$$

and say that $\mathcal{Z}$ is a matrix assignment for $\mathbf{t}$. If $\mathbf{t}$ (and therefore $\mathcal{Z}$ ) is empty, then $M_{\mathbf{t}}(\mathcal{Z}):=I_{k n}$. The matrix assignment $\mathcal{Z}$ for $\mathbf{t}$ is said to be nonsingular if the matrices assigned to the positions in $\mathbf{t}$ occupied by the 0 and $-k$ indices are nonsingular. If the matrices in $\mathcal{Z}$ are symmetric (resp. Hermitian), then $\mathcal{Z}$ is said to be a symmetric (resp. Hermitian) matrix assignment for $\mathbf{t}$.

Given an ordered list of $n \times n$ arbitrary matrices $\mathcal{Z}=\left(Z_{1}, \ldots, Z_{r}\right)$, we denote by $\operatorname{rev}(\mathcal{Z})$ the list of matrices $\left(Z_{r}, \ldots, Z_{1}\right)$. 
Given a matrix polynomial $P(\lambda)=\sum_{i=0}^{k} A_{i} \lambda^{i} \in \mathbb{F}[\lambda]^{n \times n}$, we will use the following abbreviated notation:

$$
M_{i}^{P}:=M_{i}\left(-A_{i}\right), \quad i=0: k-1
$$

and

$$
M_{-i}^{P}:=M_{-i}\left(A_{i}\right), \quad i=1: k .
$$

When the polynomial $P(\lambda)$ is understood from the context, we simply write $M_{i}$ and $M_{-i}$, instead of $M_{i}^{P}$ and $M_{-i}^{P}$ to simplify the notation.

5.2. Block-symmetric GFP. Here we recall the block-symmetric strong linearizations of a matrix polynomial $P(\lambda)$ in the family of generalized Fiedler pencils (GFP). The following GFP was introduced in [1, Theorem 3.1]:

$$
\mathcal{T}_{P}(\lambda):=\lambda M_{-1,-3, \ldots,-k+2,-k}^{P}-M_{0,2, \ldots, k-1}^{P},
$$

if $k$ is odd, and

$$
\mathcal{T}_{P}(\lambda):=\lambda M_{-1,-3, \ldots,-k+1}^{P}-M_{0,2, \ldots, k}^{P},
$$

if $k$ is even and the leading coefficient $A_{k}$ is nonsingular. The pencil $\mathcal{T}_{P}(\lambda)$ is explicitly given by

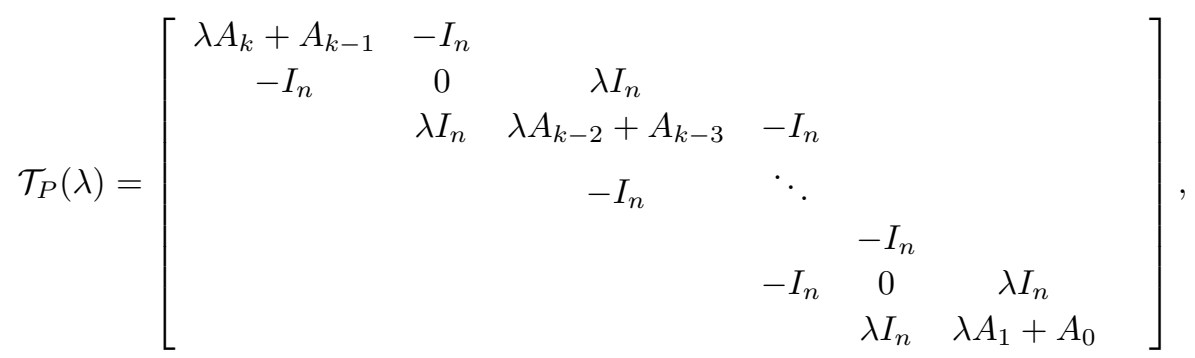

when $k$ is odd, and by

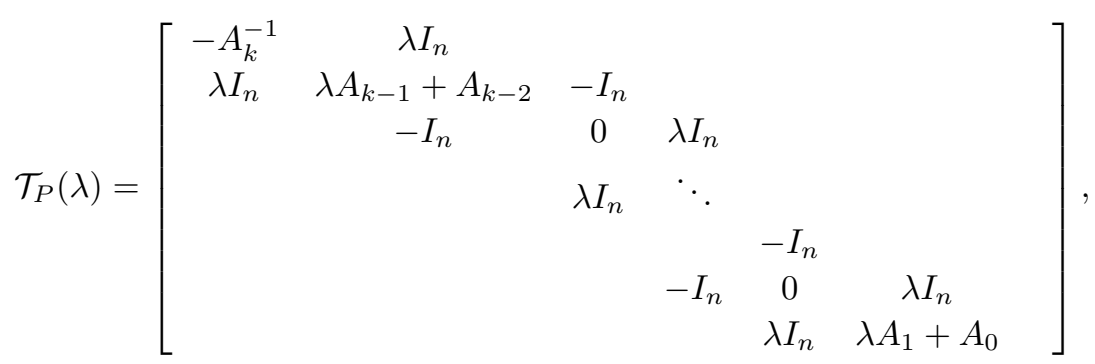

when $k$ is even and $A_{k}$ is nonsingular. We note that this pencil is not a companion form since one of its matrix coefficients contains a block equal to $A_{k}^{-1}$. Notice that $\mathcal{T}_{P}(\lambda)$ is block-symmetric, regardless of the parity of $k$. Some small variations of these pencils can be found in [23].

5.3. Block-symmetric GFPR. Next, we recall a subfamily of GFPR comprised of block-symmetric pencils.

Definition 5.10. [2] Let $P(\lambda)=\sum_{i=0}^{k} A_{i} \lambda^{i} \in \mathbb{F}[\lambda]^{n \times n}$ of degree $k$, and let $h$ be an integer such that $0 \leq h<k$. Let $\mathbf{w}_{h}$ and $k+\mathbf{v}_{h}$ be the admissible tuples associated with $h$ and $k-h-1$, respectively, and 
let $\mathbf{c}_{h}$ and $\mathbf{c}_{k-h-1}$ be the symmetric complements of $\mathbf{w}_{h}$ and $k+\mathbf{v}_{h}$, respectively. Let $\mathbf{t}_{w}$ and $k+\mathbf{t}_{v}$ be index tuples with indices from $\{0: h-1\}$ and $\{0: k-h-2\}$, respectively, such that $\left(\mathbf{t}_{w}, \mathbf{w}_{h}, \mathbf{c}_{h}, \operatorname{rev}\left(\mathbf{t}_{w}\right)\right)$ and $\left(\mathbf{t}_{v}, \mathbf{v}_{h},-k+\mathbf{c}_{k-h-1}, \operatorname{rev}\left(\mathbf{t}_{v}\right)\right)$ satisfy the SIP. Let $\mathcal{Z}_{w}$ and $\mathcal{Z}_{v}$ be matrix assignments for $\mathbf{t}_{w}$ and $\mathbf{t}_{v}$, respectively. Then, the pencil

$$
M_{\mathbf{t}_{w}, \mathbf{t}_{v}}\left(\mathcal{Z}_{w}, \mathcal{Z}_{v}\right)\left(\lambda M_{\mathbf{v}_{h}}^{P}-M_{\mathbf{w}_{h}}^{P}\right) M_{-k+\mathbf{c}_{k-h-1}, \mathbf{c}_{h}}^{P} M_{\operatorname{rev}\left(\mathbf{t}_{w}\right), \operatorname{rev}\left(\mathbf{t}_{v}\right)}\left(\operatorname{rev}\left(\mathcal{Z}_{w}\right), \operatorname{rev}\left(\mathcal{Z}_{v}\right)\right)
$$

is a block-symmetric generalized Fiedler pencil with repetition (block-symmetric GFPR) and we denote it by $L_{P}\left(h, \mathbf{t}_{w}, \mathbf{t}_{v}, \mathcal{Z}_{w}, \mathcal{Z}_{v}\right)$. If the matrix assignments $\mathcal{Z}_{w}$ and $\mathcal{Z}_{v}$ are chosen so that $M_{\mathbf{t}_{w}, \mathbf{t}_{v}}\left(\mathcal{Z}_{w}, \mathcal{Z}_{v}\right)=M_{\mathbf{t}_{w}, \mathbf{t}_{v}}^{P}$, then the block-symmetric GFPR $L_{P}\left(h, \mathbf{t}_{w}, \mathbf{t}_{v}, \mathcal{Z}_{w}, \mathcal{Z}_{v}\right)$ is a block-symmetric Fiedler pencil with repetition (block-symmetric FPR), which we denote by $L_{P}\left(h, \mathbf{t}_{w}, \mathbf{t}_{v}\right)$.

Theorem 5.11 establishes when a block-symmetric GFPR associated with a matrix polynomial $P(\lambda)$ is a strong linearization of $P(\lambda)$.

Theorem 5.11. [2, Theorem 4.9] Let $P(\lambda)=\sum_{i=0}^{k} A_{i} \lambda^{i} \in \mathbb{F}[\lambda]^{n \times n}$ be a matrix polynomial, and let $\mathcal{L}(\lambda)$ be the block-symmetric GFPR defined in (5.33). If the following three conditions hold

(i) $\mathcal{Z}_{w}$ and $\mathcal{Z}_{v}$ are nonsingular matrix assignments for $\mathbf{t}_{w}$ and $\mathbf{t}_{v}$, respectively,

(ii) $A_{0}$ is nonsingular if $h$ is odd, and

(iii) $A_{k}$ is nonsingular if $k-h$ is even,

then, the pencil $\mathcal{L}(\lambda)$ is a strong linearization of $P(\lambda)$.

Theorem 5.12 gives sufficient conditions for a block-symmetric GFPR associated with a symmetric (resp. Hermitian) matrix polynomial $P(\lambda)$ to be symmetric (resp. Hermitian).

THEOREM 5.12. [2] Let $P(\lambda)$ be a symmetric (resp. Hermitian) matrix polynomial, and let $\mathcal{L}(\lambda)$ be the block-symmetric GFPR defined in (5.33). If the matrix assignments $\mathcal{Z}_{w}$ and $\mathcal{Z}_{v}$ are symmetric (resp. Hermitian), then $\mathcal{L}(\lambda)$ is symmetric (resp. Hermitian).

6. Block-symmetric GFP and GFPR as block-symmetric block minimal bases pencils. In this section, we start by showing that the block-symmetric pencil $\mathcal{T}_{P}(\lambda)$ associated with an odd-degree matrix polynomial $P(\lambda)$ is permutationally block-congruent to the pencil $\mathcal{O}_{1}^{P}(\lambda)$. The case when $P(\lambda)$ has even degree is not considered, since $\mathcal{T}_{P}(\lambda)$ is not a companion form, that is, if the matrix coefficients of $\mathcal{T}_{P}(\lambda)$ are seen as block matrices, one of them contains a block that is not of the form $0, \pm I_{n}$ or $\pm A_{i}$. In fact, since one of the matrix coefficients of $\mathcal{T}_{P}(\lambda)$ contains a block-entry that is the inverse of $A_{k}$, the interest of this pencil in applications is very limited.

Theorem 6.1. Let $P(\lambda)=\sum_{i=0}^{k} A_{i} \lambda^{i} \in \mathbb{F}[\lambda]^{n \times n}$ be an odd-degree matrix polynomial. Let $\mathcal{T}_{P}(\lambda)$ be the block-symmetric GFP associated with $P(\lambda)$ defined in (5.32). Let $\mathbf{c}$ be the permutation of $\{1: k\}$ given by $(1,3,5, \ldots, k, 2,4, \ldots, k-1)$. Then,

$$
\left(\Pi_{\mathbf{c}}^{n}\right)^{\mathcal{B}} \mathcal{T}_{P}(\lambda) \Pi_{\mathbf{c}}^{n}=\mathcal{O}_{1}^{P}(\lambda)
$$

In other words, modulo block-permutations, the block-symmetric $G F P \mathcal{T}_{P}(\lambda)$ belongs to the family $\left\langle\mathcal{O}_{1}^{P}\right\rangle$.

Proof. Using the explicit expression for $\mathcal{T}_{P}(\lambda)$ presented in Section 5, the result is easily checked by performing the matrix product $\left(\Pi_{\mathbf{c}}^{n}\right)^{\mathcal{B}} \mathcal{T}_{P}(\lambda) \Pi_{\mathbf{c}}^{n}$.

Now we give the main result for block-symmetric GFPR associated with a matrix polynomial $P(\lambda)$, that is, we state that, up to permutations of block-rows and block-columns, every block-symmetric GFPR is in 
one of the four block-symmetric families introduced in Section 4. This result is stated in Theorem 6.2. Its proof is included in the Appendix.

Theorem 6.2. Let $P(\lambda)=\sum_{i=0}^{k} A_{i} \lambda^{i} \in \mathbb{F}[\lambda]^{n \times n}$ and let $s=(k-1) / 2$ if $k$ is odd, or $s=(k-2) / 2$ is $k$ is even. Let $L_{P}\left(h, \mathbf{t}_{w}, \mathbf{t}_{v}, \mathcal{Z}_{w}, \mathcal{Z}_{v}\right)$ be the block-symmetric GFPR associated with $P(\lambda)$ given in (5.33). Then, there exists a permutation $\mathbf{c}$ of $\{1: k\}$ such that

$$
\left(\Pi_{\mathbf{c}}^{n}\right)^{\mathcal{B}} L_{P}\left(h, \mathbf{t}_{w}, \mathbf{t}_{v}, \mathcal{Z}_{w}, \mathcal{Z}_{v}\right) \Pi_{\mathbf{c}}^{n} \in\left\{\begin{array}{l}
\left\langle\mathcal{O}_{1}^{P}\right\rangle \text { if } k \text { is odd and } h \text { is even } \\
\left\langle\mathcal{O}_{2}^{P}\right\rangle \text { if } k \text { and } h \text { are odd } \\
\left\langle\mathcal{E}_{1}^{P}\right\rangle \text { if } k \text { is even and } h \text { is odd } \\
\left\langle\mathcal{E}_{2}^{P}\right\rangle \text { if } k \text { and } h \text { are even. }
\end{array}\right.
$$

Furthermore, if the following conditions hold

(i) $\mathcal{Z}_{w}$ and $\mathcal{Z}_{v}$ are nonsingular matrix assignments for $\mathbf{t}_{w}$ and $\mathbf{t}_{v}$, respectively,

(ii) $A_{0}$ is nonsingular if $h$ is odd, and

(iii) $A_{k}$ is nonsingular if $k-h$ is even,

then $\left(\Pi_{\mathbf{c}}^{n}\right)^{\mathcal{B}} L_{P}\left(h, \mathbf{t}_{w}, \mathbf{t}_{v}, \mathcal{Z}_{w}, \mathcal{Z}_{v}\right) \Pi_{\mathbf{c}}^{n}$ is a strong linearization of $P(\lambda)$ and the following statements hold:

(a) If $\left(\Pi_{\mathbf{c}}^{n}\right)^{\mathcal{B}} L_{P}\left(h, \mathbf{t}_{w}, \mathbf{t}_{v}, \mathcal{Z}_{w}, \mathcal{Z}_{v}\right) \Pi_{\mathbf{c}}^{n} \in\left\langle\mathcal{O}_{1}^{P}\right\rangle$ is as in (4.19), then $B$ and $B^{\mathcal{B}}$ are nonsingular.

(b) If $\left(\Pi_{\mathbf{c}}^{n}\right)^{\mathcal{B}} L_{P}\left(h, \mathbf{t}_{w}, \mathbf{t}_{v}, \mathcal{Z}_{w}, \mathcal{Z}_{v}\right) \Pi_{\mathbf{c}}^{n} \in\left\langle\mathcal{O}_{2}^{P}\right\rangle$ is as in (4.24), then $E$ and $E^{\mathcal{B}}$ are nonsingular.

(c) If $\left(\Pi_{\mathbf{c}}^{n}\right)^{\mathcal{B}} L_{P}\left(h, \mathbf{t}_{w}, \mathbf{t}_{v}, \mathcal{Z}_{w}, \mathcal{Z}_{v}\right) \Pi_{\mathbf{c}}^{n} \in\left\langle\mathcal{E}_{1}^{P}\right\rangle$ is as in (4.27), then $D$ and $D^{\mathcal{B}}$ are nonsingular.

(d) If $\left(\Pi_{\mathbf{c}}^{n}\right)^{\mathcal{B}} L_{P}\left(h, \mathbf{t}_{w}, \mathbf{t}_{v}, \mathcal{Z}_{w}, \mathcal{Z}_{v}\right) \Pi_{\mathbf{c}}^{n} \in\left\langle\mathcal{E}_{2}^{P}\right\rangle$ is as in (4.30), then $D$ and $D^{\mathcal{B}}$ are nonsingular.

The following example illustrates the result for a particular block-symmetric GFPR associated with an odd degree matrix polynomial.

ExAmple 6.3. Let $P(\lambda)=\sum_{i=0}^{7} A_{i} \lambda^{i}$ be an $n \times n$ matrix polynomial of degree 7 . Consider the blocksymmetric GFPR

$$
L_{P}(\lambda):=L_{P}(k-1, \emptyset, \emptyset)=\left[\begin{array}{ccccccc}
\lambda A_{7}+A_{6} & A_{5} & -I_{n} & & & & \\
A_{5} & -\lambda A_{5}+A_{4} & \lambda I_{n} & A_{3} & -I_{n} & & \\
-I_{n} & \lambda I_{n} & 0 & 0 & 0 & & \\
& A_{3} & 0 & -\lambda A_{3}+A_{2} & \lambda I_{n} & A_{1} & -I_{n} \\
& -I_{n} & 0 & \lambda I_{n} & 0 & 0 & 0 \\
& & & A_{1} & 0 & -\lambda A_{1}+A_{0} & \lambda I_{n} \\
& & & -I_{n} & 0 & \lambda I_{n} & 0
\end{array}\right] .
$$

Let $\mathbf{c}=(1,2,4,6,3,5,7)$. Then,

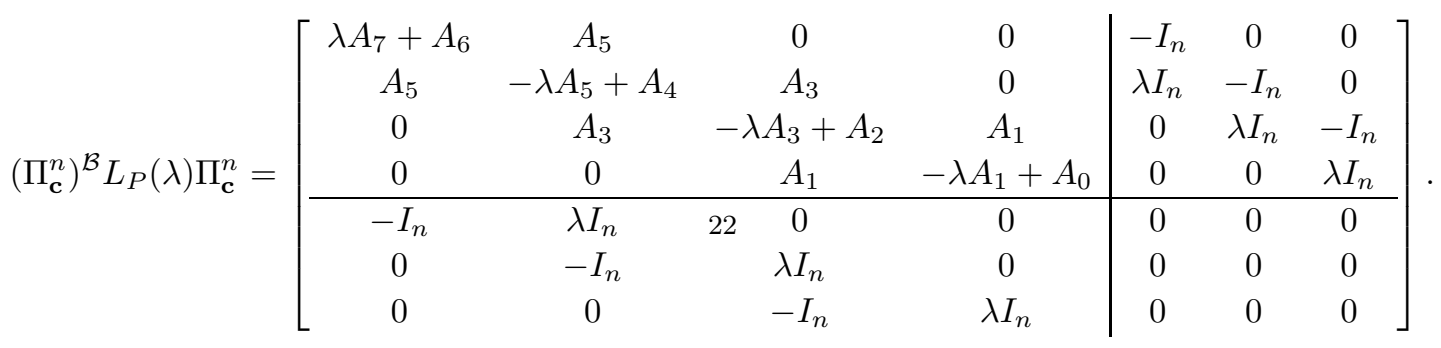


We note that $\left(\Pi_{\mathbf{c}}^{n}\right)^{\mathcal{B}} L_{P}(\lambda) \Pi_{\mathbf{c}}^{n} \in\left\langle\mathcal{O}_{1}^{P}\right\rangle$ since

$$
\left(\Pi_{\mathbf{c}}^{n}\right)^{\mathcal{B}} L_{P}(\lambda) \Pi_{\mathbf{c}}^{n}=\left[\begin{array}{cc}
I_{4 n} & C \\
0 & I_{3 n}
\end{array}\right] \mathcal{O}_{1}^{P}\left[\begin{array}{cc}
I_{4 n} & C \\
0 & I_{3 n}
\end{array}\right]^{\mathcal{B}},
$$

where

$$
C=\left[\begin{array}{ccc}
0 & 0 & 0 \\
-A_{5} & 0 & 0 \\
0 & -A_{3} & 0 \\
0 & 0 & -A_{1}
\end{array}\right]
$$

Moreover, $\left(\Pi_{\mathbf{c}}^{n}\right)^{\mathcal{B}} L_{P}(\lambda) \Pi_{\mathbf{c}}^{n}$ is a strong linearization of every matrix polynomial $P(\lambda)$.

REMARK 6.4. We note that, when $k$ is odd, by Example 4.4 and Theorem 6.1, the three pencils $D_{1}(\lambda, P)$, $D_{k}(\lambda, P)$ and $\mathcal{T}_{P}(\lambda)$ are permutationally congruent to some pencil in $\left\langle\mathcal{O}_{1}^{P}\right\rangle$. In fact, $\mathcal{T}_{P}(\lambda)$ is essentially $\mathcal{O}_{1}^{P}(\lambda)$, after permuting some block-rows and some block-columns. Thus, $\mathcal{T}_{P}(\lambda)$ could be seen, in layman's terms, as the "skeleton" of $D_{1}(\lambda, P)$ and $D_{k}(\lambda, P)$, that is, the least information that can be retained from these pencils without stopping from being a linearization of $P(\lambda)$. Hence, $\mathcal{T}_{P}(\lambda)$ is an ideal candidate to outperform numerically the combined use of $D_{1}(\lambda, P)$ and $D_{k}(\lambda, P)$ in the solution of the block-symmetric polynomial eigenvalue problem. This problem is studied in [8].

The following example gives the pencils in $\left\langle\mathcal{O}_{1}^{P}\right\rangle$ permutationally block congruent to $D_{1}(\lambda, P), D_{k}(\lambda, P)$ and $\mathcal{T}_{P}(\lambda)$, when $k=3$.

EXAmPle 6.5. Let $k=3$. Then, $D_{1}(\lambda, P)$ is permutationally block congruent to the pencil

$$
\left[\begin{array}{cc|c}
\lambda A_{3}+A_{2} & A_{1} & A_{0} \\
A_{1} & -\lambda A_{1}+A_{0} & -\lambda A_{0} \\
\hline A_{0} & -\lambda A_{0} & 0
\end{array}\right] \in\left\langle\mathcal{O}_{1}^{P}\right\rangle .
$$

The pencil $D_{k}(\lambda, P)$ is permutationally block congruent to the pencil

$$
\left[\begin{array}{cc|c}
\lambda A_{3}-A_{2} & \lambda A_{2} & -A_{3} \\
\lambda A_{2} & \lambda A_{1}-A_{0} & \lambda A_{3} \\
\hline-A_{3} & \lambda A_{3} & 0
\end{array}\right] \in\left\langle\mathcal{O}_{1}^{P}\right\rangle .
$$

The pencil $\mathcal{T}_{P}(\lambda)$ is permutationally block congruent to the pencil

$$
\mathcal{O}_{1}^{P}(\lambda)=\left[\begin{array}{cc|c}
\lambda A_{3}+A_{2} & 0 & -I_{n} \\
0 & \lambda A_{1}+A_{0} & \lambda I_{n} \\
\hline-I_{n} & \lambda I_{n} & 0
\end{array}\right]=\mathcal{O}_{1}^{P}(\lambda)
$$

7. Conclusions and future work. In this paper we have introduced four families of block-symmetric pencils that, under some generic nonsingular conditions, are block-symmetric block minimal bases pencils and strong linearizations of a matrix polynomial $P(\lambda)$. Furthermore, we have shown that every blocksymmetric GFP and block-symmetric GFPR is permutationally block-congruent to a pencil in the union of these four families, which provides an alternative approach to the implicit definition of these pencils as products of elementary matrices by providing their block structure. The importance of this result resides in the expectation that the explicit block structure of the block-symmetric GFP and GFPR will provide 
a venue to explore their numerical properties such as conditioning of eigenvalues and backward error of approximate eigenpairs. In particular, our objective is to find linearizations of $P(\lambda)$ in these famllies with optimal condition number and backward error that can replace the combined used of $D_{1}(\lambda, P)$ and $D_{k}(\lambda, P)$ when $P(\lambda)$ is symmetric or Hermitian, as suggested in the current literature.

Appendix A. (Proof of Theorem 6.2). Here we include the proof of Theorem 6.2. We start with some extra concepts and results that are necessary for this proof.

A.1. Auxiliary notation for pencils that are block-permutationally equivalent to pencils in $\left\langle\mathcal{O}_{1}^{P}\right\rangle \cup\left\langle\mathcal{E}_{1}^{P}\right\rangle$. In order to prove that all block-symmetric GFPR pencils are permutationally block-congruent to a pencil in $\left\langle\mathcal{O}_{1}^{P}\right\rangle \cup\left\langle\mathcal{O}_{2}^{P}\right\rangle \cup\left\langle\mathcal{E}_{1}^{P}\right\rangle \cup\left\langle\mathcal{E}_{2}^{P}\right\rangle$, we introduce in this section some useful concepts.

Definition A.1. [5] Let $k, n \in \mathbb{N}$. Let $\mathbf{c}=\left(c_{1}, c_{2}, \ldots, c_{k}\right)$ be a permutation of the set $\{1: k\}$. Then, we call the block-permutation matrix associated with $(\mathbf{c}, n)$, and denote it by $\Pi_{\mathbf{c}}^{n}$, the $k \times k$ block-matrix whose $\left(c_{i}, i\right)$ th block-entry is $I_{n}$, for $i=1: k$, and having $0_{n}$ in every other block-entry. In particular, we denote by $\mathbf{i d}=(1: k)$ the identity permutation.

When the scalar $n$ is clear in the context, we write $\Pi_{\mathbf{c}}$ instead of $\Pi_{\mathbf{c}}^{n}$ to simplify the notation.

An important block-permutation matrix for this paper is the so-called block standard involutory permutation matrix (block-sip matrix). Such block-permutation matrix is

$$
R_{k}:=\Pi_{(k: 1)}^{n}=\left[\begin{array}{ccc}
0 & \cdots & I_{n} \\
\vdots & . \cdot & \vdots \\
I_{n} & \cdots & 0
\end{array}\right] \in \mathbb{F}^{k n \times k n}
$$

In Definition A.2, we introduce some useful notation for pencils that are block-permutationally equivalent to pencils in $\left\langle\mathcal{O}_{1}^{P}\right\rangle$.

Definition A.2. Let $k$ be an odd positive integer, let $L(\lambda) \in \mathbb{F}[\lambda]^{k n \times k n}$ be $a k \times k$ block-pencil with block-entries of size $n \times n$, and let $s=(k-1) / 2$. Assume that there exist block-permutation matrices $\Pi_{\ell}^{n}$ and $\Pi_{\mathbf{r}}^{n}$ such that $C(\lambda):=\left(\Pi_{\ell}^{n}\right)^{\mathcal{B}} L(\lambda) \Pi_{\mathbf{r}}^{n} \in\left\langle\mathcal{O}_{1}^{P}\right\rangle$.

(a-1) We call the upper-left $(s+1) \times(s+1)$ block submatrix of $C(\lambda)$ the body of $L(\lambda)$ relative to $(\boldsymbol{\ell}, \mathbf{r})$.

(a-2) We call the body block-rows (resp. body block-columns) of $L(\lambda)$ relative to $(\boldsymbol{\ell}, \mathbf{r})$ the block-rows (resp. block-columns) of $L(\lambda)$ that, after the permutations, occupy the first $s+1$ block-rows (resp. block-columns) of $C(\lambda)$.

(b) We call the wing block-rows (resp. wing block-columns) of $L(\lambda)$ relative to $(\boldsymbol{\ell}, \mathbf{r})$ the block-rows (resp. block-columns) of $L(\lambda)$ that are not body block-rows (resp. body block-columns) relative to $(\ell, \mathbf{r})$.

The following example illustrates the concepts introduced in Definition A.2.

EXAMPLE A.3. Let us consider the following pencil

$$
L(\lambda)=\left[\begin{array}{ccccc}
\lambda A_{5}+A_{4} & A_{3} & -I_{n} & 0 & 0 \\
A_{3} & A_{2}-\lambda A_{3} & \lambda I_{n} & A_{1} & -I_{n} \\
-I_{n} & \lambda I_{n} & 0 & 0 & 0 \\
0 & A_{1} 24 & 0 & A_{0}-\lambda A_{1} & \lambda I_{n} \\
0 & -I_{n} & 0 & \lambda I_{n} & 0
\end{array}\right],
$$


which is a block-symmetric GFPR associated with the matrix polynomial $P(\lambda)=\sum_{i=0}^{5} A_{i} \lambda^{i} \in \mathbb{F}[\lambda]^{n \times n}$ (this type of block-symmetric GFPR is called the simple FPR with parameter $k-1$ in [2]). Consider the permutation $\mathbf{c}=(1,2,4,3,5)$ of $\{1: 5\}$. Then,

$$
\left(\Pi_{\mathbf{c}}^{n}\right)^{\mathcal{B}} L(\lambda) \Pi_{\mathbf{c}}^{n}=\left[\begin{array}{ccc|cc}
\lambda A_{5}+A_{4} & A_{3} & 0 & -I_{n} & 0 \\
A_{3} & A_{2}-\lambda A_{3} & A_{1} & \lambda I_{n} & -I_{n} \\
0 & A_{1} & A_{0}-\lambda A_{1} & 0 & \lambda I_{n} \\
\hline-I_{n} & \lambda I_{n} & 0 & 0 & 0 \\
0 & -I_{n} & \lambda I_{n} & 0 & 0
\end{array}\right] \in\left\langle\mathcal{O}_{1}^{P}\right\rangle .
$$

Hence,

- the first, second, and forth block-rows and block-columns of $L(\lambda)$ are, respectively, its body block-rows and body block-columns relative to $(\mathbf{c}, \mathbf{c})$; and

- the third and fifth block-rows and block-columns of $L(\lambda)$ are, respectively, its wing block-rows and wing block-columns relative to $(\mathbf{c}, \mathbf{c})$.

In Definition A.4, we introduce some useful notation for pencils that are block-permutationally equivalent to pencils in $\left\langle\mathcal{E}_{1}^{P}\right\rangle$.

Definition A.4. Let $k$ be an even positive integer, let $L(\lambda) \in \mathbb{F}[\lambda]^{k n \times k n}$ be $a k \times k$ block-pencil with block-entries of size $n \times n$, and let $s=(k-2) / 2$. Assume that there exist block-permutation matrices $\Pi_{\ell}^{n}$ and $\Pi_{\mathbf{r}}^{n}$ such that $C(\lambda):=\left(\Pi_{\ell}^{n}\right)^{\mathcal{B}} L(\lambda) \Pi_{\mathbf{r}}^{n} \in\left\langle\mathcal{E}_{1}^{P}\right\rangle$.

(a-1) We call the upper-left $(s+1) \times(s+1)$ block submatrix of $C(\lambda)$ the body of $L(\lambda)$ relative to $(\boldsymbol{\ell}, \mathbf{r})$.

(a-2) We call the body block-rows (resp. body block-columns) of $L(\lambda)$ relative to $(\boldsymbol{\ell}, \mathbf{r})$ the block-rows (resp. block-columns) of $L(\lambda)$ that, after the permutations, occupy the first $s+1$ block-rows (resp. block-columns) of $C(\lambda)$.

(b) We call the exceptional block-row (resp. exceptional block-column) of $L(\lambda)$ relative to $(\boldsymbol{\ell}, \mathbf{r})$ the block-row (resp. block-column) of $L(\lambda)$ that, after the permutations, occupies the $s+2$ block-row (resp. block-column) of $C(\lambda)$.

(c) We call the wing block-rows (resp. wing block-columns) of $L(\lambda)$ relative to $(\boldsymbol{\ell}, \mathbf{r})$ the block-rows (resp. block-columns) of $L(\lambda)$ that are not body block-rows nor exceptional block-rows (resp. body block-columns nor exceptional block-columns) relative to $(\ell, \mathbf{r})$.

We illustrate the concepts introduced in Definition A.4 in the following example.

EXAMPLE A.5. Let us consider the following pencil

$$
L(\lambda)=\left[\begin{array}{cccccc}
\lambda A_{6}+A_{5} & A_{4} & -I_{n} & 0 & 0 & 0 \\
A_{4} & A_{3}-\lambda A_{4} & \lambda I_{n} & A_{2} & -I_{n} & 0 \\
-I_{n} & \lambda I_{n} & 0 & 0 & 0 & 0 \\
0 & A_{2} & 0 & A_{1}-\lambda A_{2} & \lambda I_{n} & A_{0} \\
0 & -I_{n} & 0 & \lambda I_{n} & 0 & 0 \\
0 & 0 & 0 & A_{0} & 0 & -\lambda A_{0}
\end{array}\right],
$$

which is a block-symmetric GFPR associated with the matrix polynomial $P(\lambda)=\sum_{i=0}^{6} A_{i} \lambda^{i} \in \mathbb{F}[\lambda]^{n \times n}$ (this type of block-symmetric GFPR is called the simple FPR with parameter $k-1$ in [2]). Consider the 
permutation $\mathbf{c}=(1,2,4,6,3,5)$ of $\{1: 6\}$. Then,

$$
\left(\Pi_{\mathbf{c}}^{n}\right)^{\mathcal{B}} L(\lambda) \Pi_{\mathbf{c}}^{n}=\left[\begin{array}{ccc|c:cc}
\lambda A_{6}+A_{5} & A_{4} & 0 & 0 & -I_{n} & 0 \\
A_{4} & A_{3}-\lambda A_{4} & A_{2} & 0 & \lambda I_{n} & -I_{n} \\
-0 & A_{2} & A_{1}-\lambda A_{2} & A_{0} & 0 & \lambda I_{n} \\
\hdashline 0 & 0 & A_{0} & -\lambda A_{0} & 0 & 0 \\
\hdashline-I_{n} & \lambda I_{n} & 0 & 0 & 0 & 0 \\
0 & -I_{n} & \lambda I_{n} & 0 & 0 & 0
\end{array}\right] \in\left\langle\mathcal{E}_{1}^{P}\right\rangle .
$$

Hence,

- the first, second, and fourth block-rows and block-columns of $L(\lambda)$ are, respectively, its body blockrows and body block-columns relative to $(\mathbf{c}, \mathbf{c})$;

- the third and fifth block-rows and block-columns of $L(\lambda)$ are, respectively, its wing block-rows and wing block-columns relative to $(\mathbf{c}, \mathbf{c})$; and

- the sixth block-row and block-column of $L(\lambda)$ are, respectively, its exceptional block-row and exceptional block-column relative to $(\mathbf{c}, \mathbf{c})$.

A.2. Auxiliary definitions and lemmas for index tuples. In order to prove the auxiliary results needed in the proof of Theorem 6.2, we need to introduce some definitions and results associated with the concept of index tuple, introduced in Section 5.1.

We start by introducing a canonical form for index tuples satisfying the SIP (recall Definition 5.2). To do this, we need the following three definitions.

Definition A.6. We say that two nonnegative indices $i$ and $j$ in an index tuple commute if $|i-j| \neq 1$.

Definition A.7. [4, Definition 3.4] Given two index tuples $\mathbf{t}$ and $\mathbf{t}^{\prime}$ of nonnegative indices, we say that $\mathbf{t}$ is equivalent to $\mathbf{t}^{\prime}$ (and write $\mathbf{t} \sim \mathbf{t}^{\prime}$ ), if $\mathbf{t}=\mathbf{t}^{\prime}$ or $\mathbf{t}^{\prime}$ can be obtained from $\mathbf{t}$ by interchanging a finite number of times two distinct commuting indices in adjacent positions, that is, indices $t_{i}$ and $t_{i+1}$ such that $\left|t_{i}-t_{i+1}\right| \neq 1$ and $t_{i} \neq t_{i+1}$.

Notice that the relation $\sim$ introduced in Definition A.7 is an equivalence relation. Note, in addition, that the SIP is invariant under this relation.

REMARK A.8. It is easy to check that the commutativity relation

$$
M_{i}\left(B_{1}\right) M_{j}\left(B_{2}\right)=M_{j}\left(B_{2}\right) M_{i}\left(B_{1}\right)
$$

holds for any $n \times n$ matrices $B_{1}$ and $B_{2}$ if $\| i|-| j|| \neq 1$ and $|i| \neq|j|$. These commutativity relations readily imply that the product of elementary matrices is invariant under the equivalence relation introduced in Definition A.7, i.e., given an index tuple $\mathbf{t}$ and a matrix assignment $\mathcal{Z}$ for $\mathbf{t}$, if $\mathbf{t} \sim \mathbf{t}^{\prime}$, then $M_{\mathbf{t}}(\mathcal{Z})=M_{\mathbf{t}^{\prime}}(\tilde{\mathcal{Z}})$, where $\tilde{\mathcal{Z}}$ is the matrix assignment for $\mathbf{t}^{\prime}$ obtained from $\mathbf{t}$ by assigning to each index in $\mathbf{t}^{\prime}$ the matrix that was assigned by $\mathcal{Z}$ to the corresponding index in $\mathbf{t}$.

Definition A.9. [29, Theorem 1] Let $\mathbf{t}$ be an index tuple with indices from $\{0: h\}, h \geq 0$. Then $\mathbf{t}$ is said to be in column standard form if

$$
\mathbf{t}=\left(a_{s}: b_{s}, a_{s-1}: b_{s-1}, \ldots, a_{2}: b_{2}, a_{1}: b_{1}\right),
$$

with $h \geq b_{s}>b_{s-1}>\cdots>b_{2}>b_{1} \geq 0$ and $0 \leq a_{j} \leq b_{j}$, for all $j=1:$ s. We call each subtuple of consecutive indices $\left(a_{i}: b_{i}\right)$ a string of $\mathbf{t}$. 
The relation between the SIP, the equivalence relation of tuples, and the column standard form is stated in the following lemma.

Lemma A.10. [29, Theorem 2] Let $\mathbf{t}$ be an index tuple.

(i) If the indices of $\mathbf{t}$ are all nonnegative integers, then $\mathbf{t}$ satisfies the SIP if and only if $\mathbf{t}$ is equivalent to a tuple in column standard form.

(ii) If the indices of $\mathbf{t}$ are all negative integers and a is the minimum index in $\mathbf{t}$, then $\mathbf{t}$ satisfies the SIP if and only if $-a+\mathbf{t}$ is equivalent to a tuple in column standard form.

Two tuples in column standard form are equivalent if and only if they coincide. This motivates the following definition.

Definition A.11. [4, Definition 3.9] The unique index tuple in column standard form equivalent to an index tuple $\mathbf{t}$ of nonnegative integers satisfying the SIP is called the column standard form of $\mathbf{t}$ and is denoted by $\operatorname{csf}(\mathbf{t})$.

In the next two definitions, we pay special attention to some indices of tuples of nonnegative integers satisfying the SIP that will play a key role in the proofs of the main results in this paper.

Definition A.12. [5, Definition 4.12] For an arbitrary index tuple $\mathbf{t}$ satisfying the SIP with $\operatorname{csf}(\mathbf{t})=$ $\left(a_{s}: b_{s}, \ldots, a_{1}: b_{1}\right)$, we define heads $(\mathbf{t}):=\left\{b_{i} \mid 1 \leq i \leq s\right\}$. Furthermore, we denote by $\mathfrak{h}(\mathbf{t})$ the cardinality $s$ of heads $(\mathbf{t})$.

Given an index tuple $\mathbf{t}$, note that $\mathfrak{h}(\mathbf{t})$ gives not only the cardinality of the set heads $(\mathbf{t})$, but also the number of strings in $\operatorname{csf}(\mathbf{t})$.

Definition A.13. [5, Definition 4.13] Given an index tuple $\mathbf{t}$ and an index $x$ such that (t, $x)$ satisfies the SIP, we say that $x$ is of Type I relative to $\mathbf{t}$ if $\mathfrak{h}(\mathbf{t}, x)=\mathfrak{h}(\mathbf{t})$, and of Type II otherwise. That is, $x$ is of Type I relative to $\mathbf{t}$ if $\operatorname{csf}(\mathbf{t}, x)$ has the same number of heads (and, therefore of strings) as $\operatorname{csf}(\mathbf{t})$, and of Type II relative to $\mathbf{t}$ otherwise.

The next result relates the SIP property with the set heads $(\mathbf{t})$.

LEMma A.14. [15, Lemma 4.13] Let $h$ be a positive integer and let $\mathbf{t}$ be an index tuple with indices from $\{0: h-1\}$. Let $(a: b)$ be a string with indices from $\{0: h-2\}$. Then, the tuple $(\mathbf{t}, a: b)$ satisfies the SIP property if and only if $\mathbf{t}$ satisfies the SIP and $c \notin$ heads $(\mathbf{t})$, for all $c \in(a: b)$.

Proposition A.15. [15, Lemma 4.14] Let $\mathbf{t}=\left(a_{s}: b_{s}, \ldots, a_{2}: b_{2}, a_{1}: b_{1}\right)$ be a nonempty index tuple in column standard form with indices from $\{0: k-1\}$, for $k \geq 1$. Let $x$ be an index in $\left\{0: b_{s}-1\right\}$ such that $(\mathbf{t}, x)$ satisfies the SIP. Then, $x$ is of Type I relative to $\mathbf{t}$ if and only if $x-1 \in \operatorname{heads}(\mathbf{t})$. In particular, $x=0$ is always an index of Type II relative to $\mathbf{t}$, for every nonempty index tuple $\mathbf{t}$ in column standard form with nonnegative indices.

REMARK A.16. Using the notation of Proposition A.15, we note that this proposition implies that heads $(\mathbf{t}, x)=\left(\right.$ heads $\left.(\mathbf{t}) \backslash\left\{b_{j}\right\}\right) \cup\{x\}$ when $x$ is an index of Type I relative to $\mathbf{t}$ and $x=b_{j}+1$. Furthermore, if $x$ is of Type II relative to $\mathbf{t}$, then heads $(\mathbf{t}, x)=\operatorname{heads}(\mathbf{t}) \cup\{x\}$.

A.3. Proof of Theorem 6.2. Before we give the proof of Theorem 6.2, we need some auxiliary results for block-symmetric GFPR associated with even and odd matrix polynomials. 
A.3.1. Auxiliary results for the odd degree case. We first prove that Theorem 6.2 holds for the block-symmetric GFPR called the simple FPR with parameter $k-1$ in [2].

Theorem A.17. Let $P(\lambda)=\sum_{i=0}^{k} A_{i} \lambda^{i} \in \mathbb{F}[\lambda]^{n \times n}$ with odd degree $k$, let $s=(k-1) / 2$, and let $F_{k}(\lambda)=$ $\left(\lambda M_{-k}^{P}-M_{\mathbf{w}_{k-1}}^{P}\right) M_{\mathbf{c}_{k-1}}^{P}$. Let $\mathbf{c}$ be the permutation of $\{1: k\}$ given by $(1,2,4,6, \ldots, k-1,3,5, \ldots, k)$. Then,

$$
\left(\Pi_{\mathbf{c}}^{n}\right)^{\mathcal{B}} F_{k}(\lambda) \Pi_{\mathbf{c}}^{n}=\left[\begin{array}{c|c}
M(\lambda ; P)+C K_{s}(\lambda)+K_{s}(\lambda)^{T} C^{\mathcal{B}} & K_{s}(\lambda)^{T} \\
\hline K_{s}(\lambda) & 0
\end{array}\right] \in\left\langle\mathcal{O}_{1}^{P}\right\rangle,
$$

for some matrix $C$. Moreover, the following statements hold.

(a) The wing block-columns of $\left(\Pi_{\mathbf{c}}^{n}\right)^{\mathcal{B}} F_{k}(\lambda)$ relative to (id, c) are of the form $-e_{i} \otimes I_{n}+\lambda e_{i+1} \otimes I_{n}$, for $1 \leq i \leq s$, and are located in positions $k-j$, where $j \in\{0: k-2\}$ and $\left(\mathbf{w}_{k-1}, \mathbf{c}_{k-1}, j\right)$ satisfies the SIP.

(b) The wing block-rows of $F_{k}(\lambda) \Pi_{\mathbf{c}}^{n}$ relative to $(\mathbf{c}, \mathbf{i d})$ are of the form $-e_{i}^{T} \otimes I_{n}+\lambda e_{i+1}^{T} \otimes I_{n}$, for $1 \leq i \leq s$, and are located in positions $k-j$, where $j \in\{0: k-2\}$ and $\left(\mathbf{w}_{k-1}, \mathbf{c}_{k-1}, j\right)$ satisfies the SIP.

(c) The first block-row and the first block-column of $F_{k}(\lambda)$ are, respectively, the first body block-row and the first body block-column of $F_{k}(\lambda)$ relative to $(\mathbf{c}, \mathbf{c})$. Moreover, the block-entry of $\left(\Pi_{\mathbf{c}}^{n}\right)^{\mathcal{B}} F_{k}(\lambda) \Pi_{\mathbf{c}}^{n}$ in position $(1,1)$ equals $\lambda A_{k}+A_{k-1}$.

Furthermore, the pencil $\left(\Pi_{\mathbf{c}}^{n}\right)^{\mathcal{B}} F_{k}(\lambda) \Pi_{\mathbf{c}}^{n}$ is a strong linearization of $P(\lambda)$.

Proof. We begin by recalling the block-structure of $F_{k}(\lambda)$ when $k$ is odd [2, Section 8]. Notice that $F_{3}(\lambda)$ is partitioned to show that it is an extended block Kronecker pencil.

$$
F_{3}(\lambda)=\left[\begin{array}{cc|c}
\lambda A_{3}+A_{2} & A_{1} & -I_{n} \\
A_{1} & -\lambda A_{1}+A_{0} & \lambda I_{n} \\
\hline-I_{n} & \lambda I_{n} & 0
\end{array}\right]
$$

For $k \geq 5$ odd, we have that $F_{k}(\lambda)$ is of the form

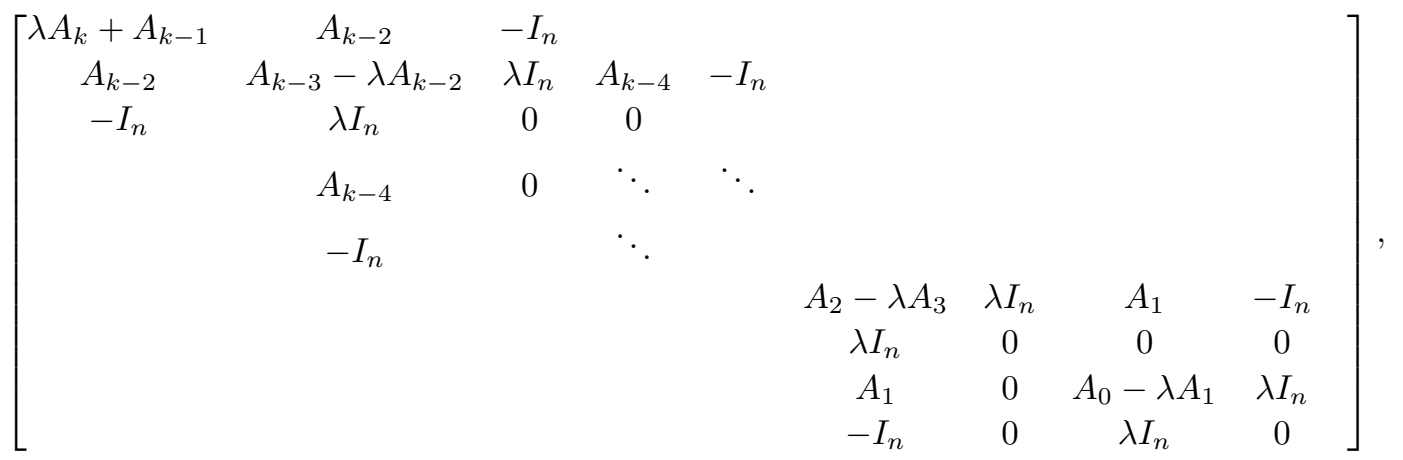

where the empty spaces denote zero blocks.

For $k=3$ the theorem can be easily checked. So let us assume $k \geq 3$. From the explicit block-structure of the pencil $F_{k}(\lambda)$ above, it is easy to see that part (c) holds and $\left(\Pi_{\mathbf{c}}^{n}\right)^{\mathcal{B}} F_{k}(\lambda) \Pi_{\mathbf{c}}^{n}$ is of the form (A.36), with

$$
C=\left[\begin{array}{cccc}
0 & 0 & \cdots & 0 \\
-A_{k-2} & 0 & \cdots & 0 \\
0 & -A_{k-4} & \cdots & 0 \\
\vdots & 2 \dot{8} & \ddots & \vdots \\
0 & 0 & \cdots & -A_{1}
\end{array}\right]
$$


Parts (a) and (b) follow from checking directly that the wing block-columns and the wing block-rows of $F_{k}(\lambda)$ relative to $(\mathbf{c}, \mathbf{c})$ are in positions $k-j \in\{3,5, \ldots, k\}$, which, in turn, by Lemmas 5.6 and A.14, correspond to those values of $j \in\{0: k-2\}$ such that $\left(\mathbf{w}_{k-1}, \mathbf{c}_{k-1}, j\right)$ satisfies the SIP. Note that $\operatorname{csf}\left(\mathbf{w}_{k-1}, \mathbf{c}_{k-1}\right)=(k-2: k-1, k-4: k-2, k-6: k-4, \ldots, 1: 3,0: 1)$.

The second step towards proving Theorem 6.2 consists in showing that this theorem holds for general block-symmetric GFPR with parameter $k-1$. We also prove some structural information concerning the block-rows and block-columns of these particular GFPR. This structural information will allow us to prove this result using an induction argument.

Theorem A.18. Let $P(\lambda)=\sum_{i=0}^{k} A_{i} \lambda^{i} \in \mathbb{F}[\lambda]^{n \times n}$ with odd degree $k$, let $s=(k-1) / 2$, and let $L_{P}(k-$ $\left.1, \mathbf{t}_{w}, \emptyset, \mathcal{Z}_{w}, \emptyset\right)$ be a block-symmetric GFPR associated with $P(\lambda)$, that is, a pencil of the form

$$
M_{\mathbf{t}_{w}}\left(\mathcal{Z}_{w}\right)\left(\lambda M_{-k}^{P}-M_{\mathbf{w}_{k-1}}^{P}\right) M_{\mathbf{c}_{k-1}}^{P} M_{\operatorname{rev}\left(\mathbf{t}_{w}\right)}\left(\operatorname{rev}\left(\mathcal{Z}_{w}\right)\right) .
$$

Then, there exists a permutation $\mathbf{c}$ of $\{1: k\}$ such that

$$
\left(\Pi_{\mathbf{c}}^{n}\right)^{\mathcal{B}} L_{P}\left(k-1, \mathbf{t}_{w}, \emptyset, \mathcal{Z}_{w}, \emptyset\right) \Pi_{\mathbf{c}}^{n}=\left[\begin{array}{c|c}
M(\lambda ; P)+C K_{s}(\lambda)+K_{s}^{T}(\lambda) C^{\mathcal{B}} & K_{s}(\lambda)^{T} B^{\mathcal{B}} \\
\hline B K_{s}(\lambda) & 0
\end{array}\right]\left\langle\left\langle\mathcal{O}_{1}^{P}\right\rangle .\right.
$$

Moreover, the following statements hold:

(a) The wing block-columns of $\left(\Pi_{\mathbf{c}}^{n}\right)^{\mathcal{B}} L_{P}\left(k-1, \mathbf{t}_{w}, \emptyset, \mathcal{Z}_{w}, \emptyset\right)$ relative to (id, $\left.\mathbf{c}\right)$ that are of the form $-e_{i} \otimes I_{n}+\lambda e_{i+1} \otimes I_{n}$, for $1 \leq i \leq s$, are located in positions $k-j$, where $j \in\{0: k-2\}$ and $\left(\mathbf{t}_{w}, \mathbf{w}_{k-1}, \mathbf{c}_{k-1}, \operatorname{rev}\left(\mathbf{t}_{w}\right), j\right)$ satisfies the SIP.

(b) The wing block-rows of $L_{P}\left(k-1, \mathbf{t}_{w}, \emptyset, \mathcal{Z}_{w}, \emptyset\right) \Pi_{\mathbf{c}}^{n}$ relative to $(\mathbf{c}, \mathbf{i d})$ that are of the form $-e_{i}^{T} \otimes$ $I_{n}+\lambda e_{i+1}^{T} \otimes I_{n}$, for $1 \leq i \leq s$, are located in positions $k-j$, where $j \in\{0: k-2\}$ and $\left(\mathbf{t}_{w}, \mathbf{w}_{k-1}, \mathbf{c}_{k-1}, \operatorname{rev}\left(\mathbf{t}_{w}\right), j\right)$ satisfies the SIP.

(c) The first block-row and the first block-column of $L_{P}\left(k-1, \mathbf{t}_{w}, \emptyset, \mathcal{Z}_{w}, \emptyset\right)$ are, respectively, the first body block-row and the first body block-column of $L_{P}\left(k-1, \mathbf{t}_{w}, \emptyset, \mathcal{Z}_{w}, \emptyset\right)$ relative to $(\mathbf{c}, \mathbf{c})$. Moreover, the block-entry of $\left(\Pi_{\mathbf{c}}^{n}\right)^{\mathcal{B}} L_{P}\left(k-1, \mathbf{t}_{w}, \emptyset, \mathcal{Z}_{w}, \emptyset\right) \Pi_{\mathbf{c}}^{n}$ in position $(1,1)$ equals $\lambda A_{k}+A_{k-1}$.

Furthermore, if $\mathcal{Z}_{w}$ is a nonsingular matrix assignment for $\mathbf{t}_{w}$, then $B$ and $B^{\mathcal{B}}$ are nonsingular, and $\left(\Pi_{\mathbf{c}}^{n}\right)^{\mathcal{B}} L_{P}\left(k-1, \mathbf{t}_{w}, \emptyset, \mathcal{Z}_{w}, \emptyset\right) \Pi_{\mathbf{c}}^{n}$ is a strong linearization of $P(\lambda)$.

Proof. We prove the result by induction on the number of indices in $\mathbf{t}_{w}$. When the tuple $\mathbf{t}_{w}$ is empty, then $L_{P}(k-1, \emptyset, \emptyset, \emptyset, \emptyset)$ is the simple FPR with parameter $k-1$ associated with $P(\lambda)$ and the result follows by Theorem A.17.

Assume that the result holds for tuples $\mathbf{t}_{w}$ with at most $\ell$ indices, with $\ell \geq 0$. Let $\mathbf{t}_{w}=\left(\mathbf{t}_{w}^{\prime}, x\right)$ be a tuple with $\ell+1$ indices, let $\mathcal{Z}_{w}=\left(\mathcal{Z}_{w}^{\prime}, Z_{x}\right)$ be a matrix assignment for $\mathbf{t}_{w}$, let $\mathcal{L}(\lambda):=L_{P}\left(k-1, \mathbf{t}_{w}, \emptyset, \mathcal{Z}_{w}, \emptyset\right)$ and $\mathcal{L}^{\prime}(\lambda):=L_{P}\left(k-1, \mathbf{t}_{w}^{\prime}, \emptyset, \mathcal{Z}_{w}^{\prime}, \emptyset\right)$. Clearly, we have $\mathcal{L}(\lambda)=M_{x}\left(Z_{x}\right) \mathcal{L}^{\prime}(\lambda) M_{x}\left(Z_{x}\right)$. Since $\mathbf{t}_{w}^{\prime}$ has $\ell$ indices, by the inductive hypothesis, there exists a block-permutation matrix $\Pi_{\sigma}$ such that

$$
\left(\Pi_{\boldsymbol{\sigma}}\right)^{\mathcal{B}} \mathcal{L}^{\prime}(\lambda) \Pi_{\boldsymbol{\sigma}}=\left[\begin{array}{c|c}
M^{\prime}(\lambda) & K_{s}(\lambda)^{T}\left(B^{\prime}\right)^{\mathcal{B}} \\
\hline B^{\prime} K_{s}(\lambda) & 0
\end{array}\right] \in\left\langle\mathcal{O}_{1}^{P}\right\rangle
$$

for some matrix $B^{\prime}$ and where $M^{\prime}(\lambda)=M(\lambda ; P)+C^{\prime} K_{s}(\lambda)+K_{s}^{T}\left(C^{\prime}\right)^{\mathcal{B}}$ for some matrix $C^{\prime}$. Moreover, properties (a), (b), and (c) hold for $\mathcal{L}^{\prime}(\lambda)$. Additionally, notice that if $\mathcal{Z}_{w}$ is a nonsingular matrix assignment for $\mathbf{t}_{w}$, then $\mathcal{Z}^{\prime}$ is a nonsingular matrix assignment for $\mathbf{t}_{w}^{\prime}$. Hence, if $\mathcal{Z}_{w}$ is a nonsingular matrix assignment 
for $\mathbf{t}_{w}$, then the matrices $B^{\prime}$ and $\left(B^{\prime}\right)^{\mathcal{B}}$ are both nonsingular, and $\left(\Pi_{\boldsymbol{\sigma}}\right)^{\mathcal{B}} \mathcal{L}^{\prime}(\lambda) \Pi_{\boldsymbol{\sigma}}$ is a strong linearization of $P(\lambda)$.

By the definition of block-symmetric GFPR, the index tuple $\left(x, \mathbf{t}_{w}^{\prime}, \mathbf{w}_{k-1}, \mathbf{c}_{k-1}, \operatorname{rev}\left(\mathbf{t}_{w}^{\prime}\right), x\right)$ satisfies the SIP, which implies that the tuple $\left(\mathbf{t}_{w}^{\prime}, \mathbf{w}_{k-1}, \mathbf{c}_{k-1}, \operatorname{rev}\left(\mathbf{t}_{w}^{\prime}\right), x\right)$ also satisfies the SIP (recall Remark 5.3). Hence, since $x \in\{0: k-2\}$ by definition of $\mathbf{t}_{w}$, by parts (a) and (b) applied to $\mathcal{L}^{\prime}(\lambda)$, the $(k-x)$ th blockcolumn of $\mathcal{L}^{\prime}(\lambda)$ is one of its wing block-columns relative to $(\boldsymbol{\sigma}, \boldsymbol{\sigma})$, and the $(k-x)$ th block-row of $\mathcal{L}^{\prime}(\lambda)$ is one of its wing block-rows relative to $(\boldsymbol{\sigma}, \boldsymbol{\sigma})$. Now we have to consider two cases.

Case I: Assume, first, that $x=0$. In this case, the action of pre- and post-multiplying $\mathcal{L}^{\prime}(\lambda)$ by $M_{0}\left(Z_{0}\right)$ consists in multiplying the $k$ th block-row and the $k$ th block-column of $\mathcal{L}^{\prime}(\lambda)$, which are, respectively, a wing block-row and a wing block- column of $\mathcal{L}^{\prime}(\lambda)$ relative to $(\boldsymbol{\sigma}, \boldsymbol{\sigma})$ by the matrix $Z_{0}$. Thus, we obtain

$$
\left(\Pi_{\boldsymbol{\sigma}}\right)^{\mathcal{B}} \mathcal{L}(\lambda) \Pi_{\boldsymbol{\sigma}}=\left[\begin{array}{c|c}
M^{\prime}(\lambda) & K_{s}(\lambda)^{T} B^{\mathcal{B}} \\
\hline B K_{s}(\lambda) & 0
\end{array}\right] \in\left\langle\mathcal{O}_{1}^{P}\right\rangle,
$$

with $B=\operatorname{diag}\left(I_{r n}, Z_{0}, I_{t n}\right) B^{\prime}$ and $B^{\mathcal{B}}=\left(B^{\prime}\right)^{\mathcal{B}} \operatorname{diag}\left(I_{r}, Z_{0}, I_{t}\right)$, for some block-identity matrices $I_{r n}$ and $I_{t n}$. Therefore, (A.38) holds with $\Pi_{\mathbf{c}}^{n}=\Pi_{\sigma}^{n}$ and $C=C^{\prime}$. Moreover, if $\mathcal{Z}_{w}$ is a nonsingular matrix assignment for $\mathbf{t}_{w}$, then $Z_{0}$ is nonsingular. Thus, in this situation, the matrices $B$ and $B^{\mathcal{B}}$ are nonsingular, and, by Theorem 4.3, the pencil $\left(\Pi_{\mathbf{c}}\right)^{\mathcal{B}} \mathcal{L}(\lambda) \Pi_{\mathbf{c}}$ is a strong linearization of $P(\lambda)$.

Now, we prove parts (a) and (b) for the case $x=0$. Since $\Pi_{\mathbf{c}}^{n}=\Pi_{\boldsymbol{\sigma}}^{n}$, the wing block-columns and the wing block-rows of $\mathcal{L}^{\prime}(\lambda)$ and $\mathcal{L}(\lambda)$ relative to $(\mathbf{c}, \mathbf{c})$ are located in the same positions. Furthermore, the wing block-columns (resp. block-rows) of $\mathcal{L}^{\prime}(\lambda)$ and $\mathcal{L}(\lambda)$ other than those in the $\mathbf{c}^{-1}(k)$ th position are equal. Since $x=0$ is a Type II index relative to $\left(\mathbf{t}_{w}^{\prime}, \mathbf{w}_{k-1}, \mathbf{c}_{k-1}, \operatorname{rev}\left(\mathbf{t}_{w}^{\prime}\right)\right)$, by Remark A.16, we also have

$$
\operatorname{heads}\left(\mathbf{t}_{w}^{\prime}, \mathbf{w}_{k-1}, \mathbf{c}_{k-1}, \operatorname{rev}\left(\mathbf{t}_{w}^{\prime}\right), 0\right)=\operatorname{heads}\left(\mathbf{t}_{w}^{\prime}, \mathbf{w}_{k-1}, \mathbf{c}_{k-1}, \operatorname{rev}\left(\mathbf{t}_{w}^{\prime}\right)\right) \cup\{0\}
$$

Thus, parts (a) and (b) follow from Lemma A.14, provided that we check that the $k$ th block-row (resp. block-column) of $L(\lambda) \Pi_{\mathbf{c}}^{n}$ (resp. $\left.\left(\Pi_{\mathbf{c}}^{n}\right)^{\mathcal{B}} L(\lambda)\right)$ is not, generically, of the form $-e_{i} \otimes I_{n}+\lambda e_{i+1} \otimes I_{n}$ (resp. $\left.-e_{i}^{T} \otimes I_{n}+\lambda e_{i+1}^{T} \otimes I_{n}\right)$, for some $1 \leq i \leq s$. Indeed, the induction hypothesis implies that the $k$ th block-row of $\mathcal{L}^{\prime}(\lambda) \Pi_{\boldsymbol{\sigma}}^{n}$ is of the form $-e_{i} \otimes I_{n}+\lambda e_{i+1} \otimes I_{n}$, which, in turn, implies that the $k$ th block-row of $L(\lambda) \Pi_{\mathbf{c}}^{n}$ is of the form $-e_{i} \otimes Z_{0}+\lambda e_{i+1} \otimes Z_{0}$. A similar argument holds for the $k$ th block-column. Thus, parts (a) and (b) are established. Part (c) follows from the induction hypothesis together with the fact that the first block-rows and first block-columns of $\mathcal{L}(\lambda)$ and $\mathcal{L}^{\prime}(\lambda)$ are, clearly, equal.

Case II: We assume, now, that $x \neq 0$. We consider two sub-cases, namely, $x$ is a Type I or a Type II index relative to the tuple $\left(\mathbf{t}_{w}^{\prime}, \mathbf{w}_{k-1}, \mathbf{c}_{k-1}, \operatorname{rev}\left(\mathbf{t}_{w}^{\prime}\right)\right)$.

Assume, first, that $x$ is a Type II index. By Proposition A.15 and Lemma A.14, $\left(\mathbf{t}_{w}^{\prime}, \mathbf{w}_{k-1}, \mathbf{c}_{k-1}\right.$, $\left.\operatorname{rev}\left(\mathbf{t}_{w}^{\prime}\right), x-1\right)$ satisfies the SIP. This, in turn, implies that the $(k-x+1)$ th block-row (resp. block-column) of $\mathcal{L}^{\prime}(\lambda)$ is one of its wing block-row (resp. block-column) relative to $(\boldsymbol{\sigma}, \boldsymbol{\sigma})$. Additionally, notice that preand post-multiplying the pencil $\mathcal{L}^{\prime}(\lambda)$ by $M_{x}\left(Z_{x}\right)$ affects only the $(k-x)$ th and $(k-x+1)$ th block-rows (resp. block-columns), which are both wing block-rows (resp. wing block-columns) of $\mathcal{L}^{\prime}(\lambda)$ relative to $(\boldsymbol{\sigma}, \boldsymbol{\sigma})$ 
by (a) and (b). Then, we can write

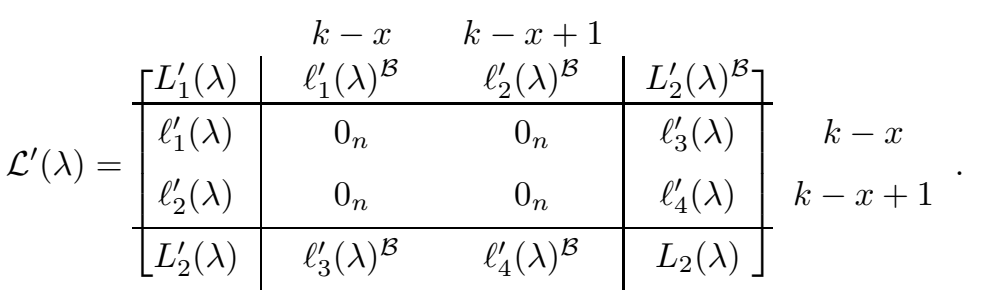

Note that, with the partition of $\mathcal{L}^{\prime}(\lambda)$ given in (A.39), the block of $\mathcal{L}^{\prime}(\lambda)$ in position $(2,2)$ is zero due to the fact that, since the $(k-x)$ th and $(k-x+1)$ th block-rows and block-columns of $\mathcal{L}^{\prime}(\lambda)$ are, respectively, wing block-rows and wing block-columns of $\mathcal{L}^{\prime}(\lambda)$ relative to $(\boldsymbol{\sigma}, \boldsymbol{\sigma})$, after being permuted the last $s$ block-entries of each of them are zero.

This implies that the pencil $\mathcal{L}(\lambda)=M_{x}\left(Z_{x}\right) \mathcal{L}^{\prime}(\lambda) M_{x}\left(Z_{x}\right)$ can be partitioned as

$\begin{array}{c|cc|c}k-x & k-x+1 & \\ L_{1}^{\prime}(\lambda) & \left(Z_{x} \ell_{1}^{\prime}(\lambda)+\ell_{2}^{\prime}(\lambda)\right)^{\mathcal{B}} & \ell_{1}^{\prime}(\lambda)^{\mathcal{B}} & L_{2}^{\prime}(\lambda)^{\mathcal{B}} \\ \hline Z_{x} \ell_{1}^{\prime}(\lambda)+\ell_{2}^{\prime}(\lambda) & 0_{n} & 0_{n} & Z_{x} \ell_{3}^{\prime}(\lambda)+\ell_{4}^{\prime}(\lambda) \\ \ell_{1}^{\prime}(\lambda) & 0_{n} & 0_{n} & \ell_{3}^{\prime}(\lambda) \\$\cline { 1 - 2 }$\left.L_{2}^{\prime}(\lambda) & \left(Z_{x} \ell_{3}^{\prime}(\lambda)+\ell_{4}^{\prime}(\lambda)\right)^{\mathcal{B}} & \ell_{3}^{\prime}(\lambda)^{\mathcal{B}} & L_{2}(\lambda)\end{array}\right] k-x+1$

where we have used the fact that the only non-zero block-entries of $\ell_{1}^{\prime}(\lambda)$ and $\ell_{3}^{\prime}(\lambda)$ are equal to $I_{n}$ or $\lambda I_{n}$ (this follows from parts (a) and (b) of the inductive hypothesis). Then, we easily obtain

$$
\left(\Pi_{\boldsymbol{\sigma}}^{n}\right)^{\mathcal{B}} \mathcal{L}(\lambda) \Pi_{\boldsymbol{\sigma}}^{n}=\left[\begin{array}{c|c}
M^{\prime}(\lambda) & K_{s}(\lambda)^{T} B^{\mathcal{B}} \\
\hline B K_{s}(\lambda) & 0
\end{array}\right] \in\left\langle\mathcal{O}_{1}^{P}\right\rangle,
$$

with $M^{\prime}(\lambda)=M(\lambda ; P)+C K_{s}(\lambda)+K_{s}^{T}(\lambda) C^{\mathcal{B}}$ and for some matrices $B$ and $B^{\mathcal{B}}$ of the form

$$
B=\left[\begin{array}{ccccc}
I_{r n} & & & & \\
& 0 & \cdots & I_{n} & \\
& & I_{t n} & & \\
& I_{n} & \cdots & Z_{x} & \\
& & & & I_{u n}
\end{array}\right] B^{\prime}, \text { and } B^{\mathcal{B}}=\left(B^{\prime}\right)^{\mathcal{B}}\left[\begin{array}{ccccc}
I_{r n} & & & & \\
& 0 & & I_{n} & \\
& \vdots & I_{t n} & \vdots & \\
& I_{n} & & Z_{x} & \\
& & & & I_{u n}
\end{array}\right]
$$

or of the form

$$
B=\left[\begin{array}{ccccc}
I_{r n} & & & & \\
& Z_{x} & \cdots & I_{n} & \\
& & I_{t n} & & \\
& 0 & \cdots & I_{n} & \\
& & & & I_{u n}
\end{array}\right] B^{\prime}, \quad \text { and } B^{\mathcal{B}}=\left(B^{\prime}\right)^{\mathcal{B}}\left[\begin{array}{ccccc}
I_{r n} & & & & \\
& Z_{x} & \cdots & I_{n} & \\
& & I_{t n} & & \\
& 0 & \cdots & I_{n} & \\
& & & & I_{u n}
\end{array}\right] \text {, }
$$

for some block-identity matrices $I_{r n}, I_{t n}$ and $I_{u n}$. Therefore, (A.38) holds with $\Pi_{\mathbf{c}}^{n}=\Pi_{\sigma}^{n}$ and $C=C^{\prime}$. Furthermore, if $\mathcal{Z}_{w}$ is a nonsingular matrix assignment for $\mathbf{t}_{w}$, then $B$ and $B^{\mathcal{B}}$ are nonsingular, since $B^{\prime}$ 
and $\left(B^{\prime}\right)^{\mathcal{B}}$ are. Recall that the elementary matrices $M_{i}(X)$, with $i \neq 0, k$ are nonsingular for all $X$. In this situation, the pencil $\left(\Pi_{\boldsymbol{\sigma}}^{n}\right)^{\mathcal{B}} \mathcal{L}(\lambda) \Pi_{\boldsymbol{\sigma}}^{n}$ is a strong linearization of $P(\lambda)$ by Theorem 4.3.

Now, we prove parts (a), (b) and (c). Recall that the $(k-x)$ th and $(k-x+1)$ th block-rows (resp. block-columns) of $\mathcal{L}^{\prime}(\lambda)$ are two of its wing block-rows (resp. wing block-columns) relative to $(\boldsymbol{\sigma}, \boldsymbol{\sigma})$. Since $\Pi_{\mathbf{c}}^{n}=\Pi_{\boldsymbol{\sigma}}^{n}$, we also have that the wing block-rows (resp. wing block-columns) of $\mathcal{L}^{\prime}(\lambda)$ and the wing blockrows (resp. wing block-columns) of $\mathcal{L}(\lambda)$ are located at the same positions. Moreover, the wing block-rows (resp. wing block-columns) of $\mathcal{L}^{\prime}(\lambda)$ and $\mathcal{L}(\lambda)$ other than those in the $(k-x)$ th and $(k-x+1)$ th positions are equal. Furthermore, the $(k-x+1)$ th block-row (resp. block-column) of $\mathcal{L}(\lambda) \Pi_{\mathbf{c}}^{n}$ (resp. of $\left(\Pi_{\mathbf{c}}^{n}\right)^{\mathcal{B}} \mathcal{L}(\lambda)$ ) is of the form $-e_{i}^{T} \otimes I_{n}+\lambda e_{i+1}^{T} \otimes I_{n}$ (resp. $-e_{i} \otimes I_{n}+\lambda e_{i+1} \otimes I_{n}$ ), for some $1 \leq i \leq s$, because, by the induction hypothesis, the $(k-x)$ th block-row (resp. block-column) of $\mathcal{L}^{\prime}(\lambda) \Pi_{\sigma}^{n}$ is of this form. Meanwhile, the $(k-x+1)$ th block-row (resp. block-column) of $\mathcal{L}(\lambda) \Pi_{\mathbf{c}}^{n}$ (resp. of $\left(\Pi_{\mathbf{c}}^{n}\right)^{\mathcal{B}} \mathcal{L}(\lambda)$ ) is clearly not of this form generically. Parts (a) and (b) follow from the preceding argument, together with

$$
\operatorname{heads}\left(\mathbf{t}_{w}^{\prime}, \mathbf{w}_{k-1}, \mathbf{c}_{w}, \operatorname{rev}\left(\mathbf{t}_{w}^{\prime}\right), x\right)=\operatorname{heads}\left(\mathbf{t}_{w}^{\prime}, \mathbf{w}_{k-1}, \mathbf{c}_{w}, \operatorname{rev}\left(\mathbf{t}_{w}^{\prime}\right)\right) \cup\{x\},
$$

which follows from Remark A.16, and Lemma A.14. To prove part (c), just notice that pre- and postmultiplication by the matrix $M_{x}\left(Z_{x}\right)$ do not affect the first block-row and the first block-column of $\mathcal{L}^{\prime}(\lambda)$, since $x \leq k-2$.

Assume, finally, that $x$ is a Type I index relative to $\left(\mathbf{t}_{w}^{\prime}, \mathbf{w}_{k-1}, \mathbf{c}_{k-1}, \operatorname{rev}\left(\mathbf{t}_{w}^{\prime}\right)\right)$. By Proposition A.15 and Lemma A.14, the tuple $\left(\mathbf{t}_{w}^{\prime}, \mathbf{w}_{k-1}, \mathbf{c}_{w}, \operatorname{rev}\left(\mathbf{t}_{w}^{\prime}\right), x-1\right)$ does not satisfy the SIP. Thus, by the inductive hypothesis, either the $(k-x+1)$ th block-row (resp. block-column) of $\mathcal{L}^{\prime}(\lambda)$ is one of its body block-rows (resp. body block-columns) relative to $(\boldsymbol{\sigma}, \boldsymbol{\sigma})$ or the $(k-x+1)$ th block-row (resp. block-column) of $\mathcal{L}^{\prime}(\lambda) \Pi_{\boldsymbol{\sigma}}^{n}$ (resp. $\left.\left(\Pi_{\boldsymbol{\sigma}}^{n}\right)^{\mathcal{B}} \mathcal{L}^{\prime}(\lambda)\right)$ is a wing block-row (resp. wing block-column) that is not of the form $-e_{i}^{T} \otimes I_{n}+\lambda e_{i+1}^{T} \otimes I_{n}$ (resp. $\quad-e_{i} \otimes I_{n}+\lambda e_{i+1} \otimes I_{n}$ ), for some $1 \leq i \leq s$. The proof that (A.38) holds in the case that the $(k-x+1)$ th block-row (resp. block-column) is a wing block-row (resp. wing block-column) is very similar to the proof for the Type II index case in the paragraphs above. So assume that the $(k-x+1)$ th block-row (resp. block-column) of $L^{\prime}(\lambda)$ is a body block-row (resp. body block-column) relative to $(\boldsymbol{\sigma}, \boldsymbol{\sigma})$. Since the $(k-x)$ th block-row (resp. block-column) of $\mathcal{L}^{\prime}(\lambda)$ is a wing block-row (resp. block-column) of $\mathcal{L}^{\prime}(\lambda)$ relative to $(\boldsymbol{\sigma}, \boldsymbol{\sigma})$, we can write

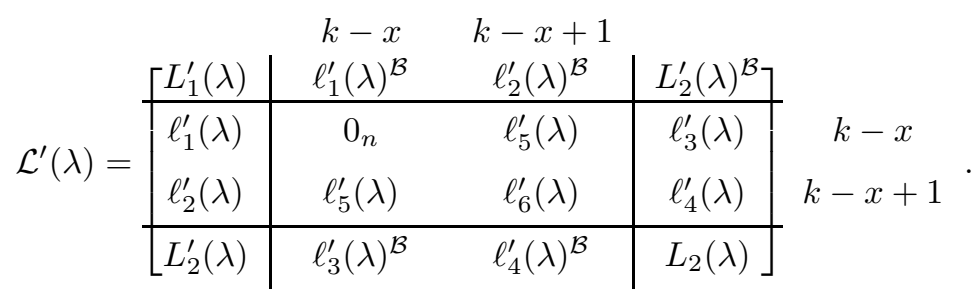

We recall again that pre- and post-multiplying the pencil $\mathcal{L}^{\prime}(\lambda)$ by $M_{x}\left(Z_{x}\right)$ affects only the $(k-x)$ th and

\begin{tabular}{|c|c|c|c|c|}
\hline$L_{1}^{\prime}(\lambda)$ & $\begin{array}{c}k-x \\
\left(Z_{x} \ell_{1}^{\prime}(\lambda)+\ell_{2}^{\prime}(\lambda)\right)^{\mathcal{B}}\end{array}$ & $\begin{array}{c}k-x+1 \\
\ell_{1}^{\prime}(\lambda)^{\mathcal{B}} \\
\end{array}$ & $L_{2}^{\prime}(\lambda)^{\mathcal{B}}$ & \\
\hline$\overline{Z_{x} \ell_{1}^{\prime}(\lambda)+\ell_{2}^{\prime}(\lambda)}$ & $\ell_{5}^{\prime}(\lambda) Z_{x}+Z_{x} \ell_{5}^{\prime}(\lambda)+\ell_{6}^{\prime}(\lambda)$ & $\ell_{5}^{\prime}(\lambda)$ & $Z_{x} \ell_{3}^{\prime}(\lambda)+\ell_{4}^{\prime}(\lambda)$ & $k-x$ \\
\hline$\ell_{1}^{\prime}(\lambda)$ & $\ell_{5}^{\prime}(\lambda)^{\mathcal{B}}$ & 0 & $\ell_{3}^{\prime}(\lambda)$ & $k-x+1$ \\
\hline$\overline{L_{2}^{\prime}(\lambda)}$ & $\left(Z_{x} \ell_{3}^{\prime}(\lambda)+\ell_{4}^{\prime}(\lambda)\right)^{\mathcal{B}}$ & $\ell_{3}^{\prime}(\lambda)^{\mathcal{B}}$ & $\overline{L_{2}(\lambda)}$ & \\
\hline
\end{tabular}
$(k-x+1)$ th block-rows (resp. block-columns). Therefore, the pencil $\mathcal{L}(\lambda)$ can be partitioned as 
where we have used the fact that the only non-zero block-entries of $\ell_{1}^{\prime}(\lambda)$ and $\ell_{3}^{\prime}(\lambda)$ are equal to $I_{n}$ or $\lambda I_{n}$ (this follows from parts (a) and (b) of the induction hypothesis). Then, setting

$$
\mathbf{d}:=(1: k-x-1, k-x+1, k-x, k-x+2: k),
$$

we easily obtain that the pencil $\left(\Pi_{\mathbf{d}}^{n}\right)^{\mathcal{B}} \mathcal{L}(\lambda) \Pi_{\mathbf{d}}^{n}$ can be partitioned as follows

\begin{tabular}{|c|c|c|c|c|}
\hline$L_{1}^{\prime}(\lambda)$ & $\begin{array}{r}k-x \\
\ell_{1}^{\prime}(\lambda)^{\mathcal{B}} \\
\end{array}$ & $\begin{array}{c}k-x+1 \\
\left(Z_{x} \ell_{1}^{\prime}(\lambda)+\ell_{2}^{\prime}(\lambda)\right)^{\mathcal{B}}\end{array}$ & $L_{2}^{\prime}(\lambda)^{\mathcal{B}}$ & \\
\hline$\ell_{1}^{\prime}(\lambda)$ & 0 & $\ell_{5}^{\prime}(\lambda)^{\mathcal{B}}$ & $\ell_{3}^{\prime}(\lambda)$ & $k-x$ \\
\hline$Z_{x} \ell_{1}^{\prime}(\lambda)+\ell_{2}^{\prime}(\lambda)$ & $\ell_{5}^{\prime}(\lambda)$ & $\ell_{5}^{\prime}(\lambda) Z_{x}+Z_{x} \ell_{5}^{\prime}(\lambda)+\ell_{6}^{\prime}(\lambda)$ & $Z_{x} \ell_{3}^{\prime}(\lambda)+\ell_{4}^{\prime}(\lambda)$ & $k-x+1$ \\
\hline$L_{2}^{\prime}(\lambda)$ & $\ell_{3}^{\prime}(\lambda)^{\mathcal{B}}$ & $\left(Z_{x} \ell_{3}^{\prime}(\lambda)+\ell_{4}^{\prime}(\lambda)\right)^{\mathcal{B}}$ & $L_{2}(\lambda)$ & \\
\hline
\end{tabular}

Thus,

$$
\left(\Pi_{\boldsymbol{\sigma}}^{n}\right)^{\mathcal{B}}\left(\Pi_{\mathbf{d}}^{n}\right)^{\mathcal{B}} \mathcal{L}(\lambda) \Pi_{\mathbf{d}}^{n} \Pi_{\boldsymbol{\sigma}}^{n}=\left[\begin{array}{c|c}
M(\lambda) & K_{s}(\lambda)^{T}\left(B^{\prime}\right)^{\mathcal{B}} \\
\hline B^{\prime} K_{s}(\lambda) & 0
\end{array}\right] \in\left\langle\mathcal{O}_{1}^{P}\right\rangle,
$$

where $M(\lambda)=M^{\prime}(\lambda)+D K_{s}(\lambda)+K_{s}(\lambda) D^{\mathcal{B}}$, for some matrix $D$. Therefore, (A.38) holds with $\Pi_{\mathbf{c}}^{n}=\Pi_{\mathbf{d}}^{n} \Pi_{\boldsymbol{\sigma}}^{n}$ and $B=B^{\prime}$. If $\mathcal{Z}_{w}$ is a nonsingular assignment for $\mathbf{t}_{w}$, then $B$ and $B^{\mathcal{B}}$ are nonsingular matrices, because $B^{\prime}$ and $\left(B^{\prime}\right)^{\mathcal{B}}$ are nonsingular, and $\left(\Pi_{\mathbf{c}}^{n}\right)^{\mathcal{B}} \mathcal{L}(\lambda) \Pi_{\mathbf{c}}^{n}$ is a strong linearization of $P(\lambda)$ by Theorem 4.3.

We finish by showing that parts (a), (b) and (c) hold. Recall that the $(k-x)$ th block-row (resp. blockcolumn) of $\mathcal{L}^{\prime}(\lambda)$ is one of its wing block-rows (resp. wing block-columns) relative to $(\boldsymbol{\sigma}, \boldsymbol{\sigma})$, and that the $(k-x+1)$ th block-row (resp. block-column) of $\mathcal{L}^{\prime}(\lambda)$ is either one of its body block-rows (resp. body block-column) or it is one of its wing block-rows (resp. wing block-columns) but not as those in part (b) (resp. part (a)). Since $\Pi_{\mathbf{c}}^{n}=\Pi_{\mathbf{d}}^{n} \Pi_{\boldsymbol{\sigma}}^{n}$, we also have that the wing block-rows (resp. wing block-columns) of $\mathcal{L}^{\prime}(\lambda)$ relative to $(\boldsymbol{\sigma}, \boldsymbol{\sigma})$ and the wing block-rows (resp. wing block-columns) of $\mathcal{L}(\lambda)$ relative to $(\mathbf{c}, \mathbf{c})$ other than those in positions $(k-x)$ th and $(k-x+1)$ th are equal and located at the same positions. Moreover, the wing block-row (resp. wing block-column) of $\mathcal{L}^{\prime}(\lambda)$ in the $(k-x)$ th position equals the wing block-row (resp. wing block-column) of $\mathcal{L}(\lambda)$ in the $(k-x+1)$ th position. Then, parts (a) and (b) follow from the preceding facts, together with

$$
\operatorname{heads}\left(\mathbf{t}_{w}^{\prime}, \mathbf{w}_{k-1}, \mathbf{c}_{k-1}, \operatorname{rev}\left(\mathbf{t}_{w}^{\prime}\right), x\right)=\left(\operatorname{heads}\left(\mathbf{t}_{w}^{\prime}, \mathbf{w}_{k-1}, \mathbf{c}_{k-1}, \operatorname{rev}\left(\mathbf{t}_{w}^{\prime}\right)\right) \cup\{x\}\right) \backslash\{x-1\},
$$

which follows from Remark A.16, and Lemma A.14. To prove part (c), just notice again that pre- and post-multiplication by the matrix $M_{x}\left(Z_{x}\right)$ do not affect the first block-row and the first block-column of $\mathcal{L}^{\prime}(\lambda)$, since $x \leq k-2$.

REMARK A.19. We note that part (c) in Theorem A.18 implies that the block-permutation $\Pi_{\mathbf{c}}^{n}$ is of the form $I_{n} \oplus \Pi_{\widetilde{\mathbf{c}}}^{n}$, for some permutation $\widetilde{\mathbf{c}}$ of the set $\{1: k-1\}$.

As a consequence of the previous theorem, we obtain Theorem A.20, which shows a structural result for another subclass of block-symmetric GFPR. In order to prove Theorem A.20, we will make use of the following immediate property of elementary matrices:

$$
R_{k} M_{-i}(B) R_{k}=M_{k-i}(B), \quad \text { for } i=1: k \text { and arbitrary } B,
$$

where $R_{k}$ is the block sip-matrix (A.34). 
Theorem A.20. Let $P(\lambda)=\sum_{i=0}^{k} A_{i} \lambda^{i} \in \mathbb{F}[\lambda]^{n \times n}$ with $k$ odd, let $s=(k-1) / 2$, and let

$$
L_{P}\left(0, \emptyset, \mathbf{t}_{v}, \emptyset, \mathcal{Z}_{v}\right)=M_{\mathbf{t}_{v}}\left(\mathcal{Z}_{v}\right)\left(\lambda M_{\mathbf{v}_{0}}^{P}-M_{0}^{P}\right) M_{-k+\mathbf{c}_{k-1}}^{P} M_{\operatorname{rev}\left(\mathbf{t}_{v}\right)}\left(\operatorname{rev}\left(\mathcal{Z}_{v}\right)\right)
$$

be a block-symmetric GFPR associated with $P(\lambda)$.

Then, there exists a permutation $\Pi_{\mathbf{c}}^{n}$ such that

$$
\left(\Pi_{\mathbf{c}}^{n}\right)^{\mathcal{B}} L_{P}\left(0, \emptyset, \mathbf{t}_{v}, \emptyset, \mathcal{Z}_{v}\right) \Pi_{\mathbf{c}}^{n}=\left[\begin{array}{c|c}
0 & B K_{s}(\lambda) \\
\hline K_{s}(\lambda)^{T} B^{\mathcal{B}} & M(\lambda ; P)+C K_{s}(\lambda)+K_{s}(\lambda)^{T} C^{\mathcal{B}}
\end{array}\right],
$$

for some matrices $B$ and $C$. Moreover, the following statements hold:

(a) The last block-column of (A.41) is the last block-row of $\left(\Pi_{\mathbf{c}}^{n}\right)^{\mathcal{B}} L_{P}\left(0, \emptyset, \mathbf{t}_{v}, \emptyset, \mathcal{Z}_{v}\right)$.

(b) The last block-row of (A.41) is the last block-column of $L_{P}\left(0, \emptyset, \mathbf{t}_{v}, \emptyset, \mathcal{Z}_{v}\right) \Pi_{\mathbf{c}}^{n}$.

(c) The block-entry of $\left(\Pi_{\mathbf{c}}^{n}\right)^{\mathcal{B}} L_{P}\left(0, \emptyset, \mathbf{t}_{v}, \emptyset, \mathcal{Z}_{v}\right) \Pi_{\mathbf{c}}^{n}$ in position $(k, k)$ equals $\lambda A_{1}+A_{0}$.

Furthermore, if $\mathcal{Z}_{v}$ is a nonsingular matrix assignment for $\mathbf{t}_{v}$, then $B$ and $B^{\mathcal{B}}$ are nonsingular.

Proof. For simplicity, let $L_{P}(\lambda):=L_{P}\left(0, \emptyset, \mathbf{t}_{v}, \emptyset, \mathcal{Z}_{v}\right)$. Let $\widehat{P}(\lambda):=-\operatorname{rev} P(\lambda)$, and let us consider the pencil $\widehat{L}(\lambda):=R_{k} \operatorname{rev}\left(-L_{P}(\lambda)\right) R_{k}$. By using (A.40) together with $R_{k}^{-1}=R_{k}$ and taking into account that the indices in the tuples $\mathbf{t}_{v}, \mathbf{v}_{0},-k+\mathbf{c}_{k-1}$ are in $\{-k:-1\}$, we obtain without much difficulty

$$
\begin{aligned}
\widehat{L}(\lambda)= & R_{k} M_{\mathbf{t}_{v}}\left(\mathcal{Z}_{v}\right) R_{k}\left(\lambda R_{k} M_{0}^{P} R_{k}-R_{k} M_{\mathbf{v}_{0}}^{P} R_{k}\right) R_{k} M_{-k+\mathbf{c}_{k-1}}^{P} R_{k} R_{k} M_{\operatorname{rev}\left(\mathbf{t}_{v}\right)}\left(\operatorname{rev}\left(\mathcal{Z}_{v}\right)\right) R_{k}= \\
& M_{\mathbf{t}_{v}+k}\left(\mathcal{Z}_{v}\right)\left(\lambda M_{-k}^{\widehat{P}}-M_{\mathbf{v}_{0}+k}^{\widehat{P}}\right) M_{\mathbf{c}_{k-1}}^{\widehat{P}} M_{\operatorname{rev}\left(\mathbf{t}_{v}\right)+k}\left(\operatorname{rev}\left(\mathcal{Z}_{v}\right)\right),
\end{aligned}
$$

which is the block-symmetric GFPR $L_{\widehat{P}}\left(k-1, \mathbf{t}_{v}+k, \emptyset, \mathcal{Z}_{v}, \emptyset\right)$ associated with $\widehat{P}(\lambda)$. Note that this pencil is of the kind of GFPR considered in Theorem A.18, because if $\left(\mathbf{t}_{v}, \mathbf{v}_{0},-k+\mathbf{c}_{k-1}, \operatorname{rev}\left(\mathbf{t}_{v}\right)\right)$ satisfies the SIP so does $\left.\left(\mathbf{t}_{v}+k, \mathbf{v}_{0}+k, \mathbf{c}_{k-1}, \operatorname{rev}\left(\mathbf{t}_{v}\right)\right)+k\right)$. Hence, by Theorem A.18, there exists a permutation $\mathbf{c}^{\prime}$ of $\{1: k\}$ such that

$$
\left(\Pi_{\mathbf{c}^{\prime}}^{n}\right)^{\mathcal{B}} \widehat{L}(\lambda) \Pi_{\mathbf{c}^{\prime}}^{n}=\left[\begin{array}{c|c}
M(\lambda ; \widehat{P})+C^{\prime} K_{s}(\lambda)+K_{s}(\lambda)^{T}\left(C^{\prime}\right)^{\mathcal{B}} & K_{s}(\lambda)^{T}\left(B^{\prime}\right)^{\mathcal{B}} \\
\hline B^{\prime} K_{s}(\lambda) & 0
\end{array}\right],
$$

for some matrices $B^{\prime}$ and $C^{\prime}$. Let $\Pi_{\mathbf{c}}^{n}:=R_{k} \Pi_{\mathbf{c}^{\prime}}^{n} R_{k}$. Taking into account that $L_{P}(\lambda)=-\operatorname{rev}\left(R_{k} \widehat{L}(\lambda) R_{k}\right)$, we, then, have

$$
\begin{aligned}
& \left(\Pi_{\mathbf{c}}^{n}\right)^{\mathcal{B}} L_{P}(\lambda) \Pi_{\mathbf{c}}^{n}=-\operatorname{rev}\left(R_{k}\left(\Pi_{\mathbf{c}^{\prime}}^{n}\right)^{\mathcal{B}} \widehat{L}(\lambda) \Pi_{\mathbf{c}^{\prime}}^{n} R_{k}\right)= \\
& -\operatorname{rev}\left(R_{k}\left[\begin{array}{c|c}
M(\lambda ; \widehat{P})+C^{\prime} K_{s}(\lambda)+K_{s}(\lambda)^{T}\left(C^{\prime}\right)^{\mathcal{B}} & K_{s}(\lambda)^{T}\left(B^{\prime}\right)^{\mathcal{B}} \\
\hline B^{\prime} K_{s}(\lambda) & 0
\end{array}\right] R_{k}\right)= \\
& {\left[\begin{array}{c|c}
0 & -\operatorname{rev}\left(R_{s} B^{\prime} K_{s}(\lambda) R_{s+1}\right) \\
\hline-\operatorname{rev}\left(R_{s} B^{\prime} K_{s}(\lambda) R_{s+1}\right)^{\mathcal{B}} & -\operatorname{rev}\left(R_{s+1}\left(M(\lambda ; \widehat{P})+C^{\prime} K_{s}(\lambda)+K_{s}(\lambda)^{T}\left(C^{\prime}\right)^{\mathcal{B}}\right) R_{s+1}\right)
\end{array}\right] .}
\end{aligned}
$$

Then, to prove the first claim of the theorem, it suffices to notice that the following two equalities hold:

$$
-\operatorname{rev}\left(R_{s} B^{\prime} K_{s}(\lambda) R_{s+1}\right)=R_{s} B^{\prime}\left(-\operatorname{rev}\left(K_{s}(\lambda) R_{s+1}\right)\right)=B K_{s}(\lambda),
$$


where $B:=R_{s} B^{\prime} R_{s}$, and,

$$
\begin{aligned}
& -\operatorname{rev}\left(R_{s+1}\left(M(\lambda ; \widehat{P})+C^{\prime} K_{s}(\lambda)+K_{s}(\lambda)^{T}\left(C^{\prime}\right)^{\mathcal{B}}\right) R_{s+1}\right) \\
& =-\operatorname{rev}\left(R_{s+1} M(\lambda ; \widehat{P}) R_{s+1}\right)+R_{s+1} C^{\prime}\left(-\operatorname{rev}\left(K_{s}(\lambda) R_{s+1}\right)\right) \\
& \quad+\left(-\operatorname{rev}\left(K_{s}(\lambda) R_{s+1}\right)\right)^{\mathcal{B}}\left(R_{s+1} C^{\prime}\right)^{\mathcal{B}} \\
& =M(\lambda ; P)+C K_{s}(\lambda)+K_{s}(\lambda)^{T} C^{\mathcal{B}}, \quad
\end{aligned}
$$

where $C:=R_{s+1} C^{\prime} R_{s}$.

Now we prove parts (a), (b), and (c). Let $M^{\prime}(\lambda):=M(\lambda ; \widehat{P})+C^{\prime} K_{s}(\lambda)+K_{s}(\lambda)^{T}\left(C^{\prime}\right)^{\mathcal{B}}$ and $M(\lambda):=$ $M(\lambda ; P)+C K_{s}(\lambda)+K_{s}(\lambda)^{T} C^{\mathcal{B}}$. Notice, first, that part (c) in Theorem A.18 implies that the first blockrow and the first block-column of the pencil $\widehat{L}(\lambda)$ are, respectively, the first body block-row and the first body block-column of the pencil $\widehat{L}(\lambda)$ relative to $\left(\mathbf{c}^{\prime}, \mathbf{c}^{\prime}\right)$, and that the block-entry in position $(1,1)$ of $M^{\prime}(\lambda)$ is $-\lambda A_{0}-A_{1}$. Since $M(\lambda)=-\operatorname{rev}\left(R_{s+1} M^{\prime}(\lambda) R_{s+1}\right)$, the block-entry in position $(s+1, s+1)$ of $M(\lambda)$ is $\lambda A_{1}+A_{0}$, which is part (c). Moreover, since $L_{P}(\lambda)=-\operatorname{rev}\left(R_{k} \widehat{L}(\lambda) R_{k}\right)$ and $\left(\Pi_{\mathbf{c}}^{n}\right)^{\mathcal{B}} L_{P}(\lambda) \Pi_{\mathbf{c}}^{n}=$ $-\operatorname{rev}\left(R_{k}\left(\Pi_{\mathbf{c}^{\prime}}^{n}\right)^{\mathcal{B}} \widehat{L}(\lambda) \Pi_{\mathbf{c}^{\prime}}^{n} R_{k}\right)$, we deduce from part (c) in Theorem A.18 that the last block-column and the last block-row of $\left(\Pi_{\mathbf{c}}^{n}\right)^{\mathcal{B}} L_{P}(\lambda) \Pi_{\mathbf{c}}^{n}$ are, respectively, the last body block-column and the last body block-row of $\left(\Pi_{\mathbf{c}}^{n}\right)^{\mathcal{B}} L_{P}(\lambda)$ and $L_{P}(\lambda) \Pi_{\mathbf{c}}^{n}$. Thus, claims (a) and (b) follow.

Finally, notice that if $\mathcal{Z}_{v}$ is a nonsingular matrix assignment for $\mathbf{t}_{v}$, then $\operatorname{rev}\left(\mathcal{Z}_{v}\right)$ is a nonsingular matrix assignment for $\operatorname{rev}\left(\mathbf{t}_{v}\right)$. Hence, if $\mathcal{Z}_{v}$ is a nonsingular matrix assignment for $\mathbf{t}_{v}$, then $B^{\prime}$ and $\left(B^{\prime}\right)^{\mathcal{B}}$ are nonsingular matrices by Theorem A.18. Thus, $B=R_{s} B^{\prime} R_{s}$ and $B^{\mathcal{B}}=R_{s}\left(B^{\prime}\right)^{\mathcal{B}} R_{s}$ are nonsingular matrices, because $R_{s}$ is nonsingular.

REMARK A.21. We note that Theorem A.20 implies that $\Pi_{\mathbf{c}}^{n}$ is of the form $\Pi_{\widetilde{\mathbf{c}}}^{n} \oplus I_{n}$, for some permutation $\widetilde{\mathbf{c}}$ of the set $\{1: k-1\}$.

A.3.2. Auxiliary results for the even degree case. We first prove that Theorem 6.2 holds for the block-symmetric GFPR called the simple FPR with parameter $k-1$ in [2].

Theorem A.22. Let $P(\lambda)=\sum_{i=0}^{k} A_{i} \lambda^{i} \in \mathbb{F}[\lambda]^{n \times n}$ of even degree $k$, let $P_{k-1}(\lambda)=\sum_{i=0}^{k-1} A_{i+1} \lambda^{i}$, let $s=(k-2) / 2$, and let $F_{k}(\lambda)=\left(\lambda M_{-k}^{P}-M_{\mathbf{w}_{k-1}}^{P}\right) M_{\mathbf{c}_{k-1}}^{P}$. Let $\mathbf{c}$ be the permutation of $\{1: k\}$ given by $(1,2,4, \ldots, k, 3,5, \ldots, k-1)$. Then,

$$
\left(\Pi_{\mathbf{c}}^{n}\right)^{\mathcal{B}} F_{k}(\lambda) \Pi_{\mathbf{c}}^{n}=\left[\begin{array}{c|c:c}
M\left(\lambda ; P_{k-1}\right)+B K_{s}(\lambda)+K_{s}(\lambda) B^{\mathcal{B}} & 0 & K_{s}(\lambda)^{T} \\
\hdashline A_{0} & \lambda A_{0} & 0 \\
\hdashline K_{s}(\lambda) & 0 & 0
\end{array}\right] \in\left\langle\mathcal{E}_{1}^{P}\right\rangle,
$$

for some matrix B. Moreover, the following statements hold:

(a) The wing block-columns of $\left(\Pi_{\mathbf{c}}^{n}\right)^{\mathcal{B}} F_{k}(\lambda)$ relative to (id, c) that are of the form $-e_{i} \otimes I_{n}+\lambda e_{i+1} \otimes I_{n}$, for $1 \leq i \leq s$, are located in positions $k-j$, where $j \in\{0: k-2\}$ and $\left(\mathbf{w}_{k-1}, \mathbf{c}_{k-1}, j\right)$ satisfies the SIP.

(b) The wing block-rows of $F_{k}(\lambda) \Pi_{\mathbf{c}}^{n}$ relative to $(\mathbf{c}, \mathbf{i d})$ that are of the form $-e_{i}^{T} \otimes I_{n}+\lambda e_{i+1}^{T} \otimes I_{n}$, for $1 \leq i \leq s$, and are located in positions $k-j$, where $j \in\{0: k-2\}$ and $\left(\mathbf{w}_{k-1}, \mathbf{c}_{k-1}, j\right)$ satisfies the SIP.

(c) The first block-row and the first block-column of $F_{k}(\lambda)$ are, respectively, the first body block-row and the first body block-column of $F_{k}(\lambda)$ relative to $(\mathbf{c}, \mathbf{c})$. Moreover, the block-entry of $\left(\Pi_{\mathbf{c}}^{n}\right)^{\mathcal{B}} F_{k}(\lambda) \Pi_{\mathbf{c}}^{n}$ in position $(1,1)$ equals $\lambda A_{k}+A_{k-1}$. 
(d) The exceptional block-column of $\left(\Pi_{\mathbf{c}}^{n}\right)^{\mathcal{B}} F_{k}(\lambda)$ relative to $(\mathbf{i d}, \mathbf{c})$ is located in position $k$, and the exceptional block-row of $F_{k}(\lambda) \Pi_{\mathbf{c}}^{n}$ relative to $(\mathbf{c}, \mathbf{i d})$ is located in position $k$.

Furthermore, if $A_{0}$ is nonsingular, the pencil $\left(\Pi_{\mathbf{c}}^{n}\right)^{\mathcal{B}} F_{k}(\lambda) \Pi_{\mathbf{c}}^{n}$ is a strong linearization of $P(\lambda)$.

Proof. We begin by recalling the block-structure of $F_{k}(\lambda)$ when $k$ is even [2, Section 8]. Notice that $F_{4}(\lambda)$ is partitioned to show that it is an extended block Kronecker pencil.

$$
F_{4}(\lambda)=\left[\begin{array}{cccc}
\lambda A_{4}+A_{3} & A_{2} & -I_{n} & 0 \\
A_{2} & -\lambda A_{2}+A_{1} & \lambda I_{n} & A_{0} \\
-I_{n} & \lambda I_{n} & 0 & 0 \\
0 & A_{0} & 0 & -\lambda A_{0}
\end{array}\right]
$$

For $k \geq 6$ even, we have that $F_{k}(\lambda)$ is of the form

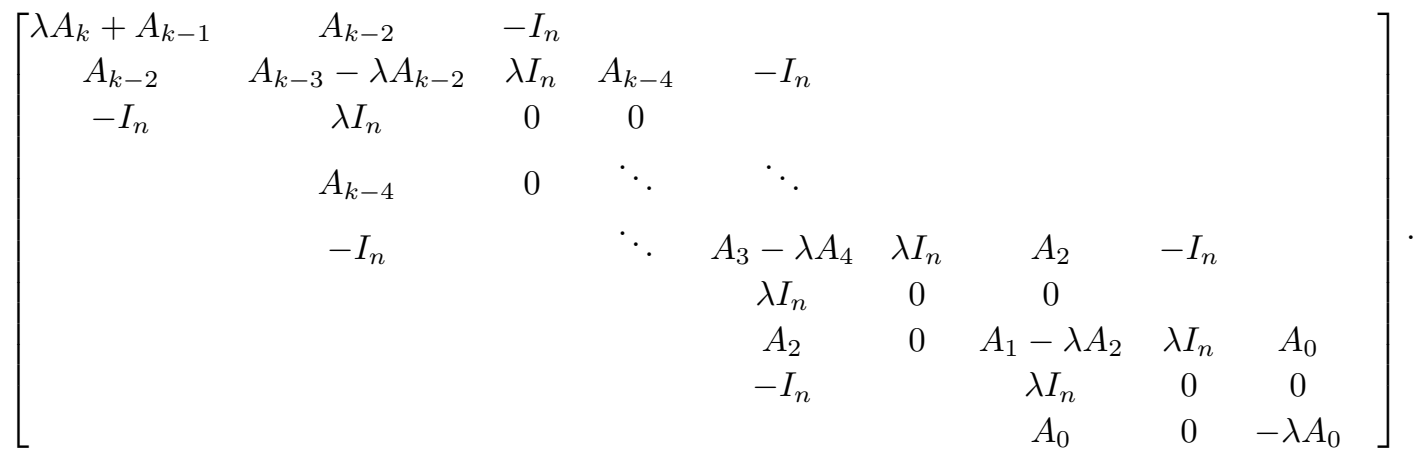

where the empty spaces denote zero blocks.

For $k=2$ or $k=4$, the theorem can be easily checked. So let us assume $k \geq 6$. From the explicit block-structure of the pencil $F_{k}(\lambda)$ it is not difficult to check that parts (c) and (d) hold and $\left(\Pi_{c}^{n}\right)^{\mathcal{B}} F_{k}(\lambda) \Pi_{c}^{n}$ is of the form (A.42), where

$$
B=\left[\begin{array}{cccc}
0 & 0 & \cdots & 0 \\
-A_{k-2} & 0 & \cdots & 0 \\
0 & -A_{k-4} & \cdots & 0 \\
\vdots & \vdots & \ddots & \vdots \\
0 & 0 & \cdots & -A_{2}
\end{array}\right]
$$

Parts (a) and (b) follow from checking directly that the wing block-columns of the form $-e_{i} \otimes I_{n}+$ $\lambda e_{i+1} \otimes I_{n}$ and the wing block-rows of $F_{k}(\lambda)$ relative to $(\mathbf{c}, \mathbf{c})$ are in positions $k-j \in\{3,5, \ldots, k-1\}$, which, in turn, by Lemmas 5.6 and A.14, correspond to those values of $j \in\{0: k-2\}$ such that $\left(\mathbf{w}_{k-1}, \mathbf{c}_{k-1}, j\right)$ satisfies the SIP. Note that $\mathbf{c s f}\left(\mathbf{w}_{k-1}, \mathbf{c}_{k-1}\right)=(k-2: k-1, k-4: k-2, \ldots, 0: 2,0)$.

Finally, from Theorem 4.11, it follows that the pencil (A.42) is a strong linearization of $P(\lambda)$ when $A_{0}$ is nonsingular.

As in the odd case, we next give the main result for some particular block-symmetric GFPR . We also prove some structural information concerning the block-rows and block-columns of these particular GFPR. 
Theorem A.23. Let $P(\lambda)=\sum_{i=0}^{k} A_{i} \lambda^{i} \in \mathbb{F}[\lambda]^{n \times n}$ of even degree $k$, let $s=(k-2) / 2$, and let $L_{P}(k-$ $\left.1, \mathbf{t}_{w}, \emptyset, \mathcal{Z}_{w}, \emptyset\right)$ be the block-symmetric GFPR associated with $P(\lambda)$ given by

$$
M_{\mathbf{t}_{w}}\left(\mathcal{Z}_{w}\right)\left(\lambda M_{-k}^{P}-M_{\mathbf{w}_{k-1}}^{P}\right) M_{\mathbf{c}_{k-1}}^{P} M_{\operatorname{rev}\left(\mathbf{t}_{w}\right)}\left(\operatorname{rev}\left(\mathcal{Z}_{w}\right)\right) .
$$

Then, there exists a block-permutation $\mathbf{c}$ of $\{1: k\}$ such that

$$
\left(\Pi_{\mathbf{c}}^{n}\right)^{\mathcal{B}} L_{P}\left(k-1, \mathbf{t}_{w}, \emptyset, \mathcal{Z}_{w}, \emptyset\right) \Pi_{\mathbf{c}}^{n} \in\left\langle\mathcal{E}_{1}^{P}\right\rangle
$$

is as in (4.27).

Moreover, the following statements hold:

(a) The wing block-columns of $\left(\Pi_{\mathbf{c}}^{n}\right)^{\mathcal{B}} L_{P}\left(k-1, \mathbf{t}_{w}, \emptyset, \mathcal{Z}_{w}, \emptyset\right)$ relative to (id, c) that are of the form $-e_{i} \otimes I_{n}+\lambda e_{i+1} \otimes I_{n}$, for $1 \leq i \leq s$, are located in positions $k-j$, where $j \in\{0: k-2\}$ and $\left(\mathbf{t}_{w}, \mathbf{w}_{k-1}, \mathbf{c}_{k-1}, \operatorname{rev}\left(\mathbf{t}_{w}\right), j\right)$ satisfies the SIP.

(b) The wing block-rows of $L_{P}\left(k-1, \mathbf{t}_{w}, \emptyset, \mathcal{Z}_{w}, \emptyset\right) \Pi_{\mathbf{c}}^{n}$ relative to $(\mathbf{c}$, id $)$ that are of the form $-e_{i}^{T} \otimes$ $I_{n}+\lambda e_{i+1}^{T} \otimes I_{n}$, for $1 \leq i \leq s$, are located in positions $k-j$, where $j \in\{0: k-2\}$ and $\left(\mathbf{t}_{w}, \mathbf{w}_{k-1}, \mathbf{c}_{k-1}, \operatorname{rev}\left(\mathbf{t}_{w}\right), j\right)$ satisfies the SIP.

(c) The first block-row and the first block-column of $L_{P}\left(k-1, \mathbf{t}_{w}, \emptyset, \mathcal{Z}_{w}, \emptyset\right)$ are, respectively, the first body block-row and the first body block-column of $L_{P}\left(k-1, \mathbf{t}_{w}, \emptyset, \mathcal{Z}_{w}, \emptyset\right)$ relative to $(\mathbf{c}, \mathbf{c})$. Moreover, the block-entry of $\left(\Pi_{\mathbf{c}}^{n}\right)^{\mathcal{B}} L_{P}\left(k-1, \mathbf{t}_{w}, \emptyset, \mathcal{Z}_{w}, \emptyset\right) \Pi_{\mathbf{c}}^{n}$ in position $(1,1)$ equals $\lambda A_{k}+A_{k-1}$.

Furthermore, if $\mathcal{Z}_{w}$ is a nonsingular matrix assignment for $\mathbf{t}_{w}$, then $C, C^{\mathcal{B}}, D$ and $D^{\mathcal{B}}$ in (4.27) are nonsingular. If, additionally, $A_{0}$ is nonsingular, $\left(\Pi_{\mathbf{c}}^{n}\right)^{\mathcal{B}} L_{P}\left(k-1, \mathbf{t}_{w}, \emptyset, \mathcal{Z}_{w}, \emptyset\right) \Pi_{\mathbf{c}}^{n}$ is a strong linearization of $P(\lambda)$.

Proof. When the tuple $\mathbf{t}_{w}$ is empty, the results follow from Theorem A.22. When the tuple $\mathbf{t}_{w}$ is not empty, the results follow by an induction argument on the number of indices in $\mathbf{t}_{w}$ almost identical to the one used for proving Theorem A.18, so we omit it.

REMARK A.24. We note that part (c) in Theorem A.23 implies that the block-permutation $\Pi_{\mathbf{c}}^{n}$ is of the form $I_{n} \oplus \Pi_{\widetilde{\mathbf{c}}}^{n}$, for some permutation $\widetilde{\mathbf{c}}$ of the set $\{1: k-1\}$.

As a consequence of Theorem A.23, we obtain Theorem A.25, which is a structural result for another subclass of block-symmetric GFPR.

Theorem A.25. Let $P(\lambda)=\sum_{i=0}^{k} A_{i} \lambda^{i} \in \mathbb{F}[\lambda]^{n \times n}$ of even degree $k$, let $s=(k-2) / 2$, and let

$$
L_{P}\left(0, \emptyset, \mathbf{t}_{v}, \emptyset, \mathcal{Z}_{v}\right)=M_{\mathbf{t}_{v}}\left(\mathcal{Z}_{v}\right)\left(\lambda M_{\mathbf{v}_{\mathbf{0}}}^{P}-M_{0}^{P}\right) M_{-k+\mathbf{c}_{k-1}}^{P} M_{\operatorname{rev}\left(\mathbf{t}_{v}\right)}\left(\operatorname{rev}\left(\mathcal{Z}_{v}\right)\right),
$$

be a block-symmetric GFPR associated with $P(\lambda)$. Then, there exists a block-permutation matrix $\Pi_{\mathbf{c}}^{n}$ such

\begin{tabular}{|c|c|c|c|}
\hline 0 & & 0 & $D K_{s}(\lambda)$ \\
\hline 0 & & $-A_{k}$ & {$\left[\begin{array}{ll}\lambda A_{k} & 0\end{array}\right]+C K_{s}(\lambda)$} \\
\hline$K_{s}(\lambda)^{T} D^{\mathcal{B}}$ & {$\left[\begin{array}{c}\lambda A_{k} \\
0\end{array}\right]$} & $+K_{s}(\lambda)^{T} C^{\mathcal{B}}$ & $M\left(\lambda ; P^{k-1}\right)+B K_{s}(\lambda)+K_{s}(\lambda)^{T} B^{\mathcal{B}}$ \\
\hline
\end{tabular}
that $\left(\Pi_{\mathbf{c}}^{n}\right)^{\mathcal{B}} L_{P}\left(0, \emptyset, \mathbf{t}_{v}, \emptyset, \mathcal{Z}_{v}\right) \Pi_{\mathbf{c}}^{n}$ is the pencil

where $P^{k-1}(\lambda)$ is defined in (4.14), for some matrices B, C, and D. Moreover, the following statements hold: 
(a) The last block-column of (A.45) is the last block-row of $\left(\Pi_{\mathbf{c}}^{n}\right)^{\mathcal{B}} L_{P}\left(0, \emptyset, \mathbf{t}_{v}, \emptyset, \mathcal{Z}_{v}\right)$.

(b) The last block-row of (A.45) is the last block-column of $L_{P}\left(0, \emptyset, \mathbf{t}_{v}, \emptyset, \mathcal{Z}_{v}\right) \Pi_{\mathbf{c}}^{n}$.

(c) The block-entry of $\left(\Pi_{\mathbf{c}}^{n}\right)^{\mathcal{B}} L_{P}\left(0, \emptyset, \mathbf{t}_{v}, \emptyset, \mathcal{Z}_{v}\right) \Pi_{\mathbf{c}}^{n}$ in position $(k, k)$ equals $\lambda A_{1}+A_{0}$.

Furthermore, if $\mathcal{Z}_{v}$ is a nonsingular matrix assignment for $\mathbf{t}_{v}$, then $C, C^{\mathcal{B}}, D$ and $D^{\mathcal{B}}$ are nonsingular. If, additionally, $A_{k}$ is nonsingular, $\left(\Pi_{\mathbf{c}}^{n}\right)^{\mathcal{B}} L_{P}\left(0, \emptyset, \mathbf{t}_{v}, \emptyset, \mathcal{Z}_{v}\right) \Pi_{\mathbf{c}}^{n}$ is a strong linearization of $P(\lambda)$.

Proof. The proof follows almost identically that of Theorem A.20, so it is only outlined. By using the reversal operation and the sip matrix $R_{k}$, together with (A.40), as it was done in the proof of Theorem A.20, the pencil $L_{P}\left(0, \emptyset, \mathbf{t}_{v}, \emptyset, \mathcal{Z}_{v}\right)$ can be transformed into one of the block-symmetric GFPR considered in Theorem A.23. Then, applying the results in Theorem A.23 to this new pencil and reversing the operations performed on $L_{P}\left(0, \emptyset, \mathbf{t}_{v}, \emptyset, \mathcal{Z}_{v}\right)$, the desired results can be obtained.

Remark A.26. We note that Theorem A.25 implies that $\Pi_{\mathbf{c}}^{n}$ is of the form $\Pi_{\tilde{\mathbf{c}}}^{n} \oplus I_{n}$, for some permutation $\widetilde{\mathbf{c}}$ of the set $\{1: k-1\}$.

A.3.3. Proof of Theorem 6.2. The main tools for proving Theorem 6.2 are Theorems A.18, A.20, A.23 and A.25, together with Lemma A.27.

Lemma A.27. Let $P(\lambda)=\sum_{i=0}^{k} A_{i} \lambda^{i} \in \mathbb{F}[\lambda]^{n \times n}$ be a matrix polynomial of degree $k$, and let $h \in\{0$ : $k-1\}$. Let $L_{P}\left(h, \mathbf{t}_{w}, \mathbf{t}_{v}, \mathcal{Z}_{w}, \mathcal{Z}_{v}\right)$ be a block-symmetric GFPR. Then, this pencil can be partitioned as

$$
L_{P}\left(h, \mathbf{t}_{w}, \mathbf{t}_{v}, \mathcal{Z}_{w}, \mathcal{Z}_{v}\right)=\left[\begin{array}{c|c|c}
D_{\mathbf{v}}(\lambda) & y_{\mathbf{v}}(\lambda) & 0 \\
\hline x_{\mathbf{v}}(\lambda) & \lambda A_{h+1}+A_{h} & x_{\mathbf{w}}(\lambda) \\
\hline 0 & y_{\mathbf{w}}(\lambda) & D_{\mathbf{w}}(\lambda)
\end{array}\right],
$$

where $D_{\mathbf{w}}(\lambda) \in \mathbb{F}[\lambda]^{n h \times n h}, D_{\mathbf{v}}(\lambda) \in \mathbb{F}[\lambda]^{n(k-h-1) \times n(k-h-1)}, x_{\mathbf{v}}(\lambda) \in \mathbb{F}[\lambda]^{n \times n(k-h-1)}, x_{\mathbf{w}}(\lambda) \in \mathbb{F}[\lambda]^{n \times n h}$, $y_{\mathbf{v}}(\lambda) \in \mathbb{F}[\lambda]^{n(k-h-1) \times n}$ and $y_{\mathbf{w}}(\lambda) \in \mathbb{F}[\lambda]^{n h \times n}$, and where the pencils

$$
F(\lambda):=\left[\begin{array}{cc}
\lambda A_{h+1}+A_{h} & x_{\mathbf{w}}(\lambda) \\
y_{\mathbf{w}}(\lambda) & D_{\mathbf{w}}(\lambda)
\end{array}\right] \quad \text { and } \quad G(\lambda):=\left[\begin{array}{cc}
D_{\mathbf{v}}(\lambda) & y_{\mathbf{v}}(\lambda) \\
x_{\mathbf{v}}(\lambda) & \lambda A_{h+1}+A_{h}
\end{array}\right]
$$

are block-symmetric GFPR associated with $Q(\lambda):=P^{h+1}(\lambda)=\lambda^{h+1} A_{h+1}+\lambda^{h} A_{h}+\cdots+\lambda A_{1}+A_{0}$ and $Z(\lambda):=P_{k-h}(\lambda)=\lambda^{k-h} A_{k}+\lambda^{k-h-1} A_{k-1}+\cdots+\lambda A_{h+1}+A_{h}$, respectively. More precisely, we have

$$
F(\lambda)=M_{\mathbf{t}_{w}}\left(\mathcal{Z}_{w}\right)\left(\lambda M_{-h-1}^{Q}-M_{\mathbf{w}_{h}}^{Q}\right) M_{\mathbf{c}_{h}}^{Q} M_{\mathrm{rev}\left(\mathbf{t}_{w}\right)}\left(\operatorname{rev}\left(\mathcal{Z}_{w}\right)\right)
$$

and

$$
G(\lambda)=M_{\mathbf{t}_{v}}\left(\mathcal{Z}_{v}\right)\left(\lambda M_{\mathbf{v}_{h}}^{Z}-M_{0}^{Z}\right) M_{\mathbf{c}_{-k+h}}^{Z} M_{\operatorname{rev}\left(\mathbf{t}_{v}\right)}\left(\operatorname{rev}\left(\mathcal{Z}_{v}\right)\right) .
$$

Proof. The result follows by combining the partition in [5, Lemma 4.34] with Theorems A.20 and A.25, which imply that the upper-left block-entry and the bottom-right block-entry of, respectively, $F(\lambda)$ and $G(\lambda)$ are both equal to $\lambda A_{h+1}+A_{h}$.

We are finally in a position to prove Theorem 6.2.

Proof. (of Theorem 6.2) We have to distinguish four cases, namely, (i) $k$ odd and $h$ even; (ii) $k$ and $h$ odd; (iii) $k$ even and $h$ odd; and (iv) $k$ and $h$ even. The main ideas and steps for proving Theorem 6.2 in each of these four cases are the same. For this reason, we only prove the most difficult case, which turns out to be case (ii). The proofs for the remaining cases are just outlined at the end, leaving the details to the interested reader. 
Let us assume that $k$ and $h$ are odd. For simplicity, instead of writing $L_{P}\left(h, \mathbf{t}_{w}, \mathbf{t}_{v}, \mathcal{Z}_{w}, \mathcal{Z}_{v}\right)$, we write $L_{p}(\lambda)$. The first goal, then, is to show that there exists a block-permutation matrix $\Pi_{\mathbf{c}}^{n}$ such that

$$
\left(\Pi_{\mathbf{c}}^{n}\right)^{\mathcal{B}} L_{P}(\lambda) \Pi_{\mathbf{c}}^{n} \in\left\langle\mathcal{O}_{2}^{P}\right\rangle .
$$

By Lemma A.27 applied to the pencil $L_{P}(\lambda)$, we can partition $L_{p}(\lambda)$ as in (A.46), where the pencil

$$
F(\lambda):=\left[\begin{array}{cc}
\lambda A_{h+1}+A_{h} & x_{\mathbf{w}}(\lambda) \\
y_{\mathbf{w}}(\lambda) & D_{\mathbf{w}}(\lambda)
\end{array}\right]=M_{\mathbf{t}_{w}}\left(\mathcal{Z}_{w}\right)\left(\lambda M_{-h-1}^{Q}-M_{\mathbf{w}_{h}}^{Q}\right) M_{\mathbf{c}_{w}} M_{\operatorname{rev}\left(\mathbf{t}_{w}\right)}\left(\operatorname{rev}\left(\mathcal{Z}_{w}\right)\right)
$$

is a block-symmetric GFPR associated with the matrix polynomial $Q(\lambda):=\lambda^{h+1} A_{h+1}+\lambda^{h} A_{h}+\cdots+\lambda A_{1}+A_{0}$, and where the pencil

$$
G(\lambda):=\left[\begin{array}{cc}
D_{\mathbf{v}}(\lambda) & y_{\mathbf{v}}(\lambda) \\
x_{\mathbf{v}}(\lambda) & \lambda A_{h+1}+A_{h}
\end{array}\right]=M_{\mathbf{t}_{v}}\left(\mathcal{Z}_{v}\right)\left(\lambda M_{\mathbf{v}_{h}}^{Z}-M_{0}^{Z}\right) M_{\mathbf{c}_{v}} M_{\operatorname{rev}\left(\mathbf{t}_{v}\right)}\left(\operatorname{rev}\left(\mathcal{Z}_{v}\right)\right)
$$

is a block-symmetric GFPR associated with the matrix polynomial $Z(\lambda):=\lambda^{k-h} A_{k}+\lambda^{k-h-1} A_{k-1}+\cdots+$ $\lambda A_{h+1}+A_{h}$. Notice that $F(\lambda)$ is one of the block-symmetric GFPR considered in Theorem A.23, while $G(\lambda)$ is one of the block-symmetric GFPR considered in Theorem A.25.

Let $s_{1}=(h-1) / 2$ and $s_{2}=(k-h-2) / 2$. From Theorem A.23, together with Remark A.24 and Definition 4.10, we obtain that there exists a block-permutation matrix $I_{n} \oplus \Pi_{\mathbf{c}_{1}}^{n}$ such that $\left(I_{n} \oplus \Pi_{\mathbf{c}_{1}}^{n}\right)^{\mathcal{B}} F(\lambda)\left(I_{n} \oplus \Pi_{\mathbf{c}_{1}}^{n}\right)=$

$$
\left[\begin{array}{c|c:c}
M\left(\lambda ; Q_{h}\right)+B K_{s_{1}}(\lambda)+K_{s_{1}}(\lambda) B^{\mathcal{B}} & {\left[\begin{array}{c}
0 \\
A_{0}
\end{array}\right]+K_{s_{1}}(\lambda)^{T} C^{\mathcal{B}}} & K_{s_{1}}(\lambda)^{T} D^{\mathcal{B}} \\
\hdashline[0 & -\lambda A_{0} & 0 \\
\hdashline D K_{s_{1}}(\lambda) & 0 & 0
\end{array}\right]
$$

\begin{tabular}{|c|c|c|c|}
\hline 0 & & 0 & $D^{\prime} K_{s_{2}}(\lambda)$ \\
\hline 0 & & $-A_{k}$ & {$\left[\begin{array}{ll}\lambda A_{k} & 0\end{array}\right]+C^{\prime} K_{s_{2}}(\lambda)$} \\
\hline$K_{s_{2}}(\lambda)^{T}\left(D^{\prime}\right)^{\mathcal{B}}$ & {$\left[\begin{array}{c}\lambda A_{k} \\
0\end{array}\right.$} & $+K_{s_{2}}(\lambda)^{T}\left(C^{\prime}\right)^{\mathcal{B}}$ & $M\left(\lambda ; Z^{k-h-1}\right)+B^{\prime} K_{s_{2}}(\lambda)+K_{s_{2}}(\lambda)^{T}\left(B^{\prime}\right)^{\mathcal{B}}$ \\
\hline
\end{tabular}

for some matrices $B, C$ and $D$. Additionally, from Theorem A.25, together with Remark A.26, we obtain that there exists a block-permutation matrix $\Pi_{\mathbf{c}_{2}}^{n} \oplus I_{n}$ such that $\left(\Pi_{\mathbf{c}_{2}}^{n} \oplus I_{n}\right)^{\mathcal{B}} G(\lambda)\left(\Pi_{\mathbf{c}_{2}}^{n} \oplus I_{n}\right)=$

for some matrices $B^{\prime}, C^{\prime}$, and $D^{\prime}$.

Next, let us introduce the notation

$$
\left[T_{s}(\lambda), t_{s}(\lambda)\right]:=\left[\begin{array}{c|ccc:c}
-I_{n} & \lambda I_{n} & 0 & 0 & \\
& -I_{n} & \lambda I_{n} & & \\
& \ddots & \ddots & \\
& & -I_{n} & \lambda I_{n}
\end{array}\right]=:\left[r_{s}(\lambda) \mid R_{s}(\lambda)\right]
$$

where $t_{s}(\lambda)$ and $r_{s}(\lambda)$ are of size $s n \times n$. We also introduce the following notation

$$
M\left(\lambda ; Q_{h}\right)+B K_{s_{1}}(\lambda)+K_{s_{1}}(\lambda) B^{\mathcal{B}}=:\left[\begin{array}{cc}
\lambda A_{h+1}+A_{h} & m_{1}^{Q}(\lambda) \\
m_{1}^{Q}(\lambda)^{\mathcal{B}} & \widehat{M}^{Q}(\lambda)
\end{array}\right]
$$


and

$$
M\left(\lambda ; Z^{k-h-1}\right)+B^{\prime} K_{s_{2}}(\lambda)+K_{s_{2}}(\lambda)^{T}\left(B^{\prime}\right)^{\mathcal{B}}=:\left[\begin{array}{cc}
\widehat{M}^{Z}(\lambda) & m_{1}^{Z}(\lambda) \\
m_{1}^{Z}(\lambda)^{\mathcal{B}} & \lambda A_{h+1}+A_{h}
\end{array}\right] .
$$

Then, omitting the dependence on $\lambda$ for lack of space, notice that the pencil $\left(\Pi_{\mathbf{c}_{2}}^{n} \oplus I_{n} \oplus \Pi_{\mathbf{c}_{1}}\right)^{\mathcal{B}} L_{P}(\lambda)\left(\Pi_{\mathbf{c}_{2}}^{n} \oplus\right.$ $\left.I_{n} \oplus \Pi_{\mathbf{c}_{1}}\right)=$

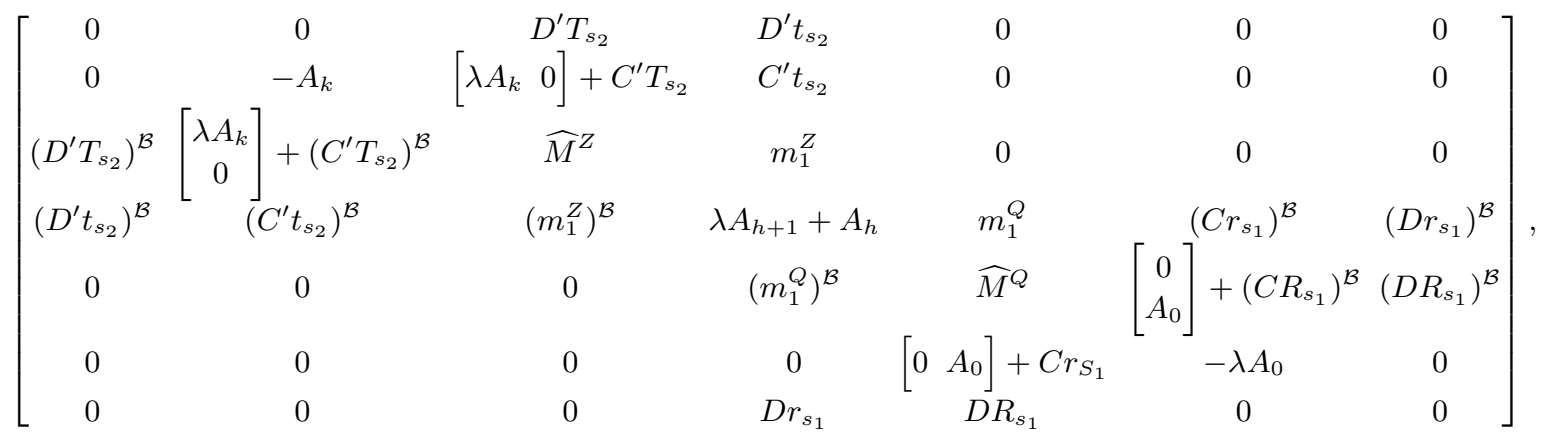

is block-permutationally congruent to

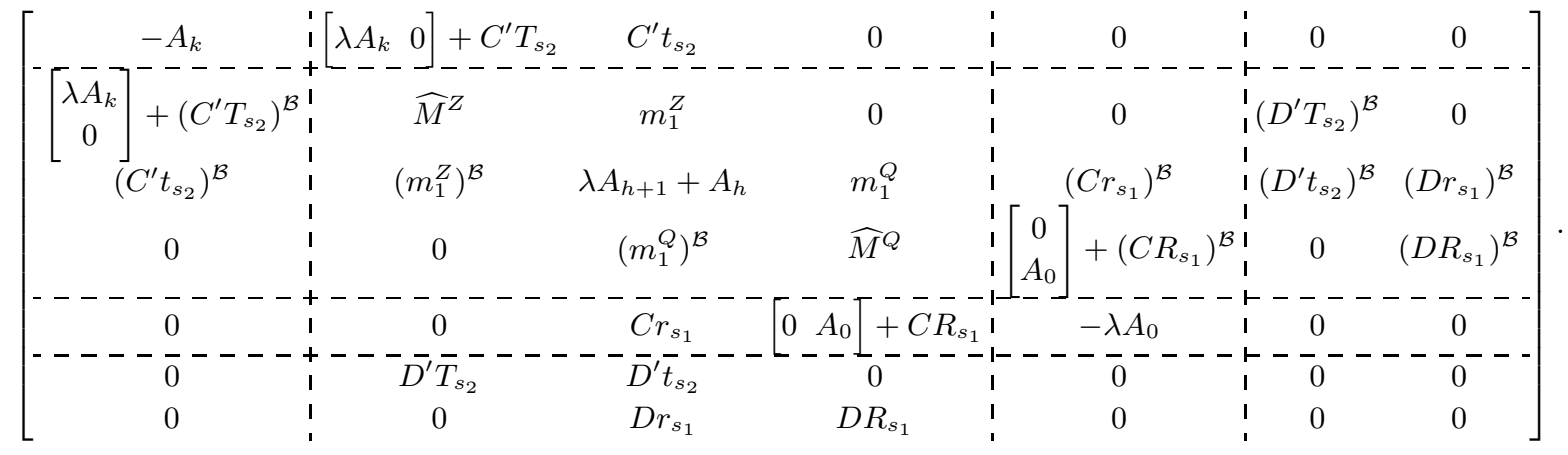

To finish the proof, it suffices to check that the above pencil belongs to $\left\langle\mathcal{O}_{2}^{P}\right\rangle$. Hence, we have to analyze the different blocks highlighted by the dash lines. Let $s=(k-1) / 2$. First, notice

$$
\left[\begin{array}{ccc}
D^{\prime} T_{s_{2}}(\lambda) & D^{\prime} t_{s_{2}}(\lambda) & 0 \\
0 & D r_{s_{1}}(\lambda) & D R_{s_{1}}(\lambda)
\end{array}\right]=\left[\begin{array}{cc}
D^{\prime} & 0 \\
0 & D
\end{array}\right] K_{s-1}(\lambda)=: \widetilde{D} K_{s-1}(\lambda)
$$

Second, notice

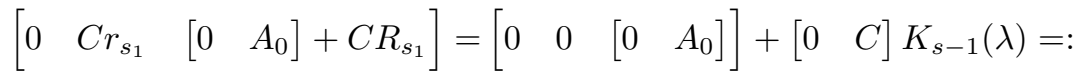

$$
\begin{aligned}
& {\left[\begin{array}{ll}
0 & A_{0}
\end{array}\right]+\widetilde{C} K_{s-1}(\lambda) \text {. }}
\end{aligned}
$$

Third, notice

$$
\begin{aligned}
& \left.\left.\left[\begin{array}{ll}
\lambda A_{k} & 0
\end{array}\right]+C^{\prime} T_{s_{2}}(\lambda) \quad C^{\prime} t_{s_{2}}(\lambda) \quad 0\right]=\left[\begin{array}{ll}
\lambda A_{k} & 0
\end{array}\right] \quad 0 \quad 0\right]+\left[\begin{array}{ll}
C^{\prime} & 0
\end{array}\right] K_{s-1}(\lambda)=: \\
& {\left[\begin{array}{ll}
\lambda A_{k} & 0
\end{array}\right]+\widetilde{C}^{\prime} K_{s-1}(\lambda) \text {. }}
\end{aligned}
$$


Finally, writing $B=\left[\begin{array}{c}b \\ \widehat{B}\end{array}\right]$ and $B^{\prime}=\left[\begin{array}{c}\widehat{B}^{\prime} \\ b^{\prime}\end{array}\right]$, where $b$ and $b^{\prime}$ are, respectively, the first and last block-rows of $B$ and $B^{\prime}$, it is not difficult to check that

$$
\begin{aligned}
& {\left[\begin{array}{ccc}
\widetilde{M}^{Z}(\lambda) & m_{1}^{Z}(\lambda) & 0 \\
m_{1}^{Z}(\lambda)^{\mathcal{B}} & \lambda A_{h+1}+A_{h} & m_{1}^{Q}(\lambda) \\
0 & m_{1}^{Q}(\lambda)^{\mathcal{B}} & \widehat{M}^{Q}(\lambda)
\end{array}\right]=M\left(\lambda ; P_{k-1}^{k-1}\right)+\left[\begin{array}{cc}
\widehat{B}^{\prime} & 0 \\
b^{\prime} & b \\
0 & B
\end{array}\right] K_{s-1}(\lambda)+} \\
& K_{s-1}(\lambda)^{T}\left[\begin{array}{ccc}
\left(\widehat{B}^{\prime}\right)^{\mathcal{B}} & \left(b^{\prime}\right)^{\mathcal{B}} & 0 \\
0 & b^{\mathcal{B}} & B^{\mathcal{B}}
\end{array}\right]=: \\
& M\left(\lambda ; P_{k-1}^{k-1}\right)+\widetilde{B} K_{s-1}(\lambda)+K_{s-1}(\lambda)^{T}(\widetilde{B})^{\mathcal{B}} .
\end{aligned}
$$

Thus, we have proven that there exists a block-permutation matrix $\Pi_{\mathbf{c}}^{n}$ such that the permuted GFPR $\left(\Pi_{\mathbf{c}}^{n}\right)^{\mathcal{B}} L_{p}(\lambda) \Pi_{\mathbf{c}}^{n}$ is of the form

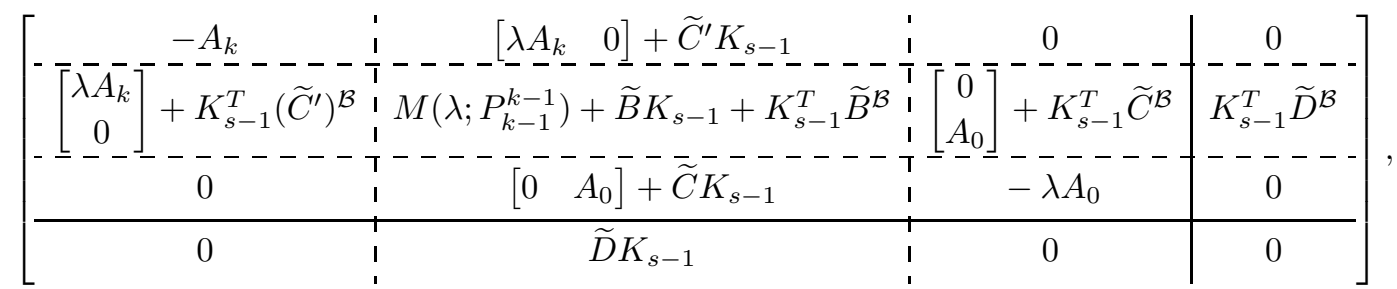

which belongs to $\left\langle\mathcal{O}_{2}^{P}\right\rangle$. Furthermore, if statement (i) holds, then the matrices $D, D^{\mathcal{B}}, D^{\prime}$ and $\left(D^{\prime}\right)^{\mathcal{B}}$ in (A.48) are nonsingular by Theorems A.23 and A.23. Hence, in this situation, the matrices $\widetilde{D}$ and $\widetilde{D}^{\mathcal{B}}$ are nonsingular. Then, taking into consideration the statements (ii) and (iii), by Theorem 4.7, the pencil $\left(\Pi_{\mathbf{c}}^{n}\right)^{\mathcal{B}} L_{p}(\lambda) \Pi_{\mathbf{c}}^{n}$ is a strong linearization of $P(\lambda)$.

For proving Theorem 6.2 in cases (a), (c) and (d), one may proceed as follow. First, one apply Lemma A.27 to the block-symmetric GFPR in order to "split" the pencil into two simpler block-symmetric GFPR $F(\lambda)$ and $G(\lambda)$, as we have done at the beginning of the proof for case (b). Depending on the parity of $k$ and $h$, these simpler GFPR are as one of those considered in Theorems A.18, A.20, A.23 or A.25. Applying the corresponding theorems to $F(\lambda)$ and $G(\lambda)$, and a simple block-permutation, one easily obtain the desired result.

\section{REFERENCES}

[1] E. N. Antoniou and S. Vologiannidis. A new family of companion forms of polynomial matrices. Electron. J. Linear Algebra, 11 (2004), 78-87.

[2] M. I. Bueno, F. M. Dopico, and S. Furtado. Linearizations of Hermitian matrix polynomials preserving the sign characteristic. SIAM J. Matrix Anal. Appl., 38 (2017), 73-101.

[3] M. I. Bueno, K. Curlett and S. Furtado. Structured strong linearizations from Fiedler pencils with repetition I. Linear Algebra Appl., 460 (2014), 51-80.

[4] M. I. Bueno, F. M. Dopico, S. Furtado and M. Rychnovsky. Large vector spaces of block-symmetric strong linearizations of matrix polynomials. Linear Algebra Appl., 477 (2015), 165-210.

[5] M. Bueno, F. M. Dopico, J. Pérez, R. Saavedra, and B. Zykoski. A unified approach to Fiedler-like pencils via strong block minimal bases pencils. Submitted for publication (2016). Also available as arXiv:1611.07170.

[6] M. I. Bueno and S. Furtado. Palindromic linearizations of a matrix polynomial of odd degree obtained from Fiedler pencils with repetition. Electron. J. Linear Algebra, 23 (2012), 562-577.

[7] M. I. Bueno and S. Furtado. Structured linearizations from Fiedler pencils with repetition II. Linear Algebra Appl., 463 (2014), 282-321. 
[8] M. I. Bueno, F. M. Dopico, S. Furtado and L. Medina. Block-symmetric linearizations of odd degree matrix polynomials with optimal condition number and backward error. Preprint.

[9] M. I. Bueno, F. M. Dopico, S. Furtado and L. Medina. Block-symmetric linearizations of even degree matrix polynomials with optimal condition number and backward error. In preparation.

[10] F. De Terán, F. M. Dopico, and D. S. Mackey. Linearizations of singular matrix polynomials and the recovery of minimal indices. Electron. J. Linear Algebra, 18 (2009), 371-402.

[11] F. De Terán, F. M. Dopico and D. S. Mackey. Fiedler companion linearizations and the recovery of minimal indices. SIAM J. Matrix Anal. Appl., 31 (2010), 2181-2204.

[12] F. De Terán, F. M. Dopico and D. S. Mackey. Palindromic companion forms for matrix polynomials of odd degree. J. Comput. Appl. Math., 236 (2011), 1464-1480.

[13] F. De Terán, F. M. Dopico and D. S. Mackey. Fiedler companion linearizations for rectangular matrix polynomials. Linear Algebra Appl., 437 (2012), 957-991.

[14] F. De Terán, F. M. Dopico and D. S. Mackey. Spectral equivalence of matrix polynomials and the Index Sum Theorem. Linear Algebra Appl., 459 (2014), 264-333.

[15] F. M. Dopico, P. W. Lawrence, J. Pérez and P. Van Dooren. Block Kronecker linearizations of matrix polynomials and their backward errors. Submitted for publication (2016). Available as MIMS EPrint 2016.34.

[16] H. Faßbender and P. Saltenberger. Block-Kronecker ansatz spaces for matrix polynomials. Linear Algebra Appl., in press. https://doi.org/10.1016/j.laa.2017.03.019

[17] G. D. Forney, Jr. Minimal bases of rational vector spaces, with applications to multivariable linear systems. SIAM J. Control, 13 (1975), 493-520.

[18] F. R. Gantmatcher. The Theory of Matrices. AMS Chelsea, Providence, RI, 1998.

[19] N. J. Higham, D. S. Mackey, and F. Tisseur, The conditioning of linearizations of matrix polynomials., SIAM J. Matrix Anal. Appl., 28 (2006), 1005-1028.

[20] N. Higham, D. S. Mackey, N. Mackey and F. Tisseur. Symmetric linearizations for matrix polynomials. SIAM J. Matrix Anal. Appl., 29(1) (2006), 143-159.

[21] D. S. Mackey, N. Mackey, C. Mehl, and V. Mehrmann, Vector spaces of linearizations for matrix polynomials, SIAM J. Matrix Anal. Appl., 28(4): 971-1004, 2006.

[22] D. S. Mackey, N. Mackey, C. Mehl and V. Mehrmann. Structured polynomial eigenvalue problems: good vibrations from good linearizations. SIAM J. Matrix Anal. Appl., 28 (2006), 1029-1051.

[23] D. S. Mackey, N. Mackey, C. Mehl, and V. Mehrmann. Jordan structures of alternating matrix polynomials. Linear Algebra Appl., 432 (2010), 971-1004.

[24] C. B. Moler and G. W. Stewart. An algorithm for generalized matrix eigenvalue problems. SIAM J. Numer. Anal., 10(2) (1971), 241-256.

[25] L. Robol, R. Vandebril, and P. Van Dooren. A framework for structured linearizations of matrix polynomials in various bases. SIAM J. Matrix Anal. Appl., 38(1) (2017), 188-216.

[26] F. Tisseur. Backward error and condition of polynomial eigenvalue problems. Linear Algebra Appl., 309 (2000), 339-361.

[27] P. Van Dooren. The computation of Kronecker's canonical form of a singular pencil. Linear Algebra Appl., 27 (1979), 103-140.

[28] P. Van Dooren and P. Dewilde. The eigenstructure of an arbitrary polynomial matrix: computational aspects. Linear Algebra Appl., 50 (1983), 545-579.

[29] S. Vologiannidis and E. N. Antoniou. A permuted factors approach for the linearization of polynomial matrices. Math. Control Signals Syst., 22 (2011), 317-342. 